\title{
Direct Construction of 4-Hydroxybenzils via para-Selective C-C Bond Coupling of Phenols and Aryl Methyl Ketones
}

Jia-Chen Xiang, Yan Cheng, Miao Wang, Yan-Dong $\mathrm{Wu}^{\mathrm{a} *}$, and $\mathrm{An}-\mathrm{Xin} \mathrm{Wu}^{\mathrm{b}}$ * Key Laboratory of Pesticide \& Chemical Biology, Ministry of Education, Central China Normal University, Wuhan 430079, P. R. China

āE-mail: .chwuyd@mail.ccnu.edu.cn.

bE-mail: .chwuax@mail.ccnu.edu.cn

\section{Table of Contents page}

1. General pp.S2

2. General procedure for the synthesis pp.S2

3. Control experiments and remarks pp.S3-S8

4. Characterization data for target compound pp.S9-20

5. Crystallographic data and molecular structure pp.S21-23

6. Copies of 'H NMR, "C NMR Spectra pp.S24-57 


\section{General}

All substrates and reagents were commercially available and used without further purification. TLC analysis was performed using pre-coated glass plates. Column chromatography was performed using alkaline aluminum oxide (200-300 mesh). IR spectra were recorded on a Perkin-Elmer PE-983 infrared spectrometer as $\mathrm{KBr}$ pellets with absorption in $\mathrm{cm}^{-1} .1 \mathrm{H}$ spectra were recorded in $\mathrm{CDCl}_{3}$ or DMSO- $d 6$ or $\mathrm{CD}_{3} \mathrm{COCD}_{3}$ or $\mathrm{CNCD}_{3}$ or acetic acid- $d 6$ on $300 / 400 / 600 \mathrm{MHz}$ NMR spectrometers and resonances $(\delta)$ are given in parts per million relative to tetramethylsilane. Data are reported as follows: chemical shift, multiplicity $(\mathrm{s}=$ singlet, $\mathrm{d}=$ doublet, $\mathrm{t}=$ triplet, $\mathrm{m}=$ multiplet $\mathrm{q}=$ quadruple), coupling constants $(\mathrm{Hz})$ and integration. ${ }^{13} \mathrm{C}$ spectra were recorded in $\mathrm{CDCl}_{3}$ or DMSO- $d_{6}$ or $\mathrm{CD}_{3} \mathrm{COCD}_{3}$ or $\mathrm{CNCD}_{3}$ or acetic acid- $d 6$ on $100 / 150 \mathrm{MHz}$. ${ }^{19} \mathrm{~F}$ spectra were recorded in DMSO- $d 6$ on $376 \mathrm{MHz}$. NMR spectrometers and resonances $(\delta)$ are given in ppm. HRMS were obtained on a Bruker 7-tesla FT-ICR MS equipped with an electrospray source. The X-ray crystal structure determinations were obtained on a Bruker SMART APEX CCD system. Melting points were determined using XT-4 apparatus and not corrected. Optical rotation was measured with polarimeter: Atopol IV (an average value of three times parallel tests).

\section{General procedure for the synthesis}

\section{$>$ for 3 (3aa as an example)}

A mixture of acetophenone 1a $(1.0 \mathrm{mmol}), o$-cresol $\mathbf{2 a}(2.0 \mathrm{mmol})$, hydriodic acid, $57 \mathrm{wt} . \%$ solution in $\mathrm{H}_{2} \mathrm{O}(0.5 \mathrm{mmol})$, boric acid $(2.0 \mathrm{mmol})$, in DMSO $(3.0 \mathrm{~mL})$ was stirred at $115{ }^{\circ} \mathrm{C}$ for 6 hours in a pressure vessel. Then added $50 \mathrm{~mL}$ water and $30 \mathrm{~mL}$ saturated brine solution to the mixture and extracted with EtOAc 3 times $(3 \times 50 \mathrm{~mL})$. The extract was washed with $10 \%$ $\mathrm{Na}_{2} \mathrm{~S}_{2} \mathrm{O}_{3}$ solution, dried over anhydrous $\mathrm{Na}_{2} \mathrm{SO}_{4}$ and concentrated under reduced pressure. The crude product was purified by column chromatography (eluent: petroleum ether /EtOAc=10/1) to afford the product 3aa as yellow oil.

\section{$>$ for NSC 115566}

A mixture of acetophenone $1 \mathrm{a}(1.0 \mathrm{mmol})$, phenol $(2.0 \mathrm{mmol})$, hydriodic acid, $57 \mathrm{wt} . \%$ solution in $\mathrm{H}_{2} \mathrm{O}(0.5 \mathrm{mmol})$, boric acid $(2.0 \mathrm{mmol})$, in DMSO $(3.0 \mathrm{~mL})$ was stirred at $115{ }^{\circ} \mathrm{C}$ for 6 hours in a pressure vessel. Then added $50 \mathrm{~mL}$ water and $30 \mathrm{~mL}$ saturated brine solution to the mixture and extracted with EtOAc 3 times $(3 \times 50 \mathrm{~mL})$. The extract was washed with $10 \% \mathrm{Na}_{2} \mathrm{~S}_{2} \mathrm{O}_{3}$ solution, dried over anhydrous $\mathrm{Na}_{2} \mathrm{SO}_{4}$ and concentrated under reduced pressure. The product was purified by column chromatography (eluent: petroleum ether $/ \mathrm{EtOAc}=10 / 1$ ) to afford the crude product 1-(4-hydroxyphenyl)-2-phenylethane-1,2-dione. Then without further purification, the crude product was treated with 1-bromopropane (1.0 equiv), $\mathrm{K}_{2} \mathrm{CO}_{3}$ (1.0 equiv), $\mathrm{CH}_{3} \mathrm{CN}$ (3.0 mL), the mixture was reflux for $4 \mathrm{~h}$, then concentrated under reduced pressure. The product was purified by column chromatography (eluent: petroleum ether /EtOAc=15/1) to afford NSC 115566 as white solid.

\section{$>$ for gram-scale synthesis of 3kl}

A mixture of 1-(4-methoxyphenyl)ethanone $1 \mathbf{k}(10.0 \mathrm{mmol})$, resorcinol $(10.0 \mathrm{mmol})$, hydriodic acid, 57 wt.\% solution in $\mathrm{H}_{2} \mathrm{O}(5.0 \mathrm{mmol})$, boric acid $(20.0 \mathrm{mmol})$, in DMSO $(20.0 \mathrm{~mL})$ was stirred at $115{ }^{\circ} \mathrm{C}$ for 8 hours in a pressure vessel. Then added $500 \mathrm{~mL}$ water and $300 \mathrm{~mL}$ saturated brine solution to the mixture and extracted with EtOAc 3 times $(3 \times 150 \mathrm{~mL})$. The extract was 
washed with $10 \% \mathrm{Na}_{2} \mathrm{~S}_{2} \mathrm{O}_{3}$ solution, dried over anhydrous $\mathrm{Na}_{2} \mathrm{SO}_{4}$ and concentrated under reduced pressure. The crude product was purified by column chromatography (eluent: petroleum ether /EtOAc=10/1 and $\left.\mathrm{CH}_{2} \mathrm{Cl}_{2} / \mathrm{MeOH}=4 / 1\right)$ to afford the product 3kl as yellow solid. The gram-scale synthesis of $\mathbf{3 j} \mathbf{j}$ underwent a similar process.

\section{$>$ for Sophodibenzoside $\mathbf{F}$}

To a solution of 3kl (136 mg, $0.5 \mathrm{mmol})$ and glucuronate bromide $(369 \mathrm{mg}, 0.9 \mathrm{mmol})$ in $\mathrm{CH}_{3} \mathrm{CN}(10.0 \mathrm{~mL})$ was added $\mathrm{Ag}_{2} \mathrm{O}(173 \mathrm{mg}, 0.75 \mathrm{mmol})$ under Ar protection. After stirring for 5 $\mathrm{h}$ at $35^{\circ} \mathrm{C}$, the mixture was concentrated. The residue was applied to chromatography over a short column of silica gel (petroleum ether : EtOAc $=4: 1$ ) to give crude product as a yellow solid. Then treated with $\mathrm{KOH}(0.5$ equiv) in $\mathrm{MeOH}(5.0 \mathrm{~mL})$ at room temperature for $0.5 \mathrm{~h}$. The mixture was filtered with washing with EtOAc. The filtrates were concentrated under vacuo to give a residue, which was purified by silica gel column chromatography $\left(\mathrm{CH}_{2} \mathrm{Cl}_{2}: \mathrm{MeOH}=4: 1\right)$ to afford Sophodibenzoside F as a yellow solid. The synthesis of Sophodibenzoside I underwent a similar process. 


\section{Control experiments and remarks}

\subsection{Spectra for ${ }^{18} \mathrm{O}$-labeling experiments}

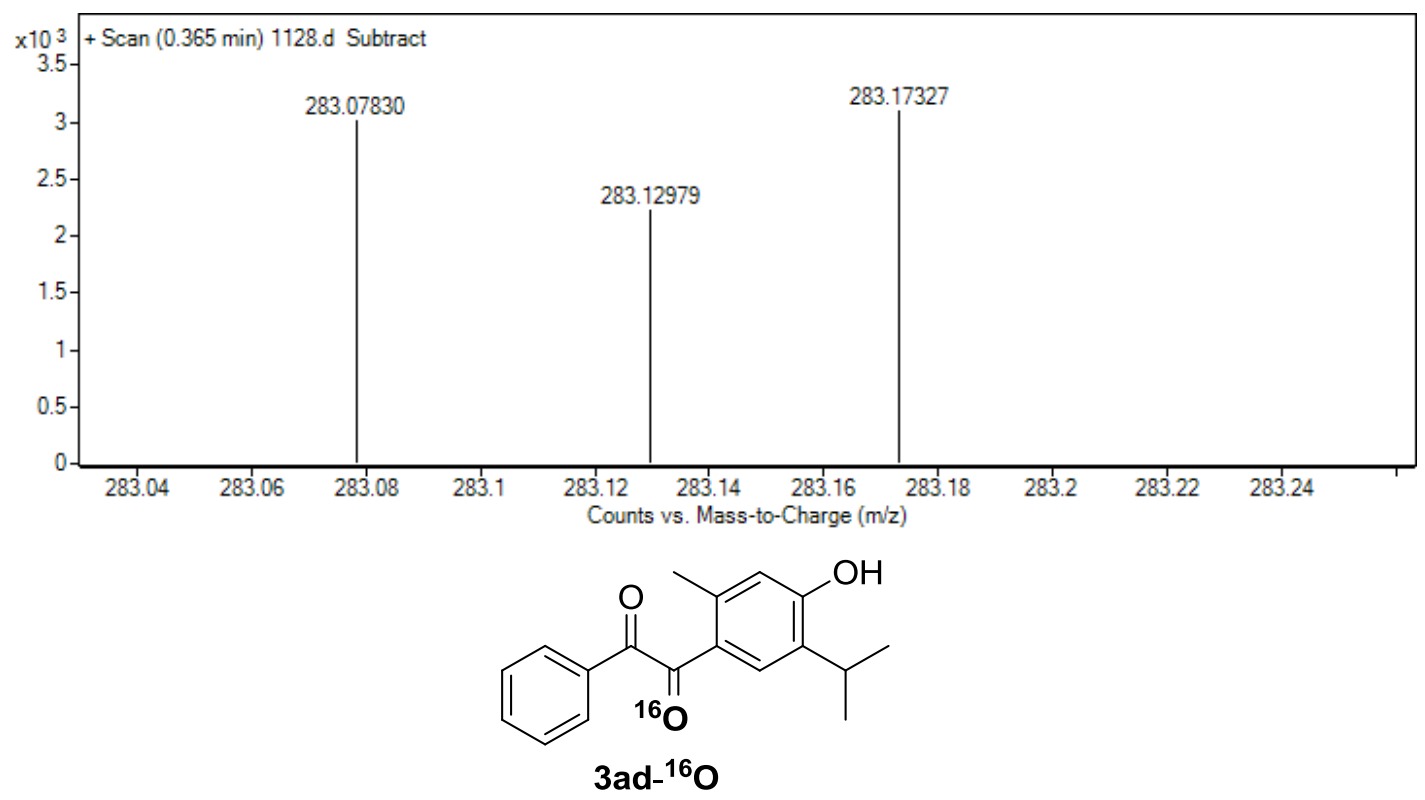

HRMS (ESI): $\mathrm{m} / \mathrm{z}[\mathrm{M}+\mathrm{H}]^{+}$calcd for $\mathrm{C}_{18} \mathrm{H}_{19}{ }^{16} \mathrm{O}_{3}: 283.1329$; found: 283.1298 .

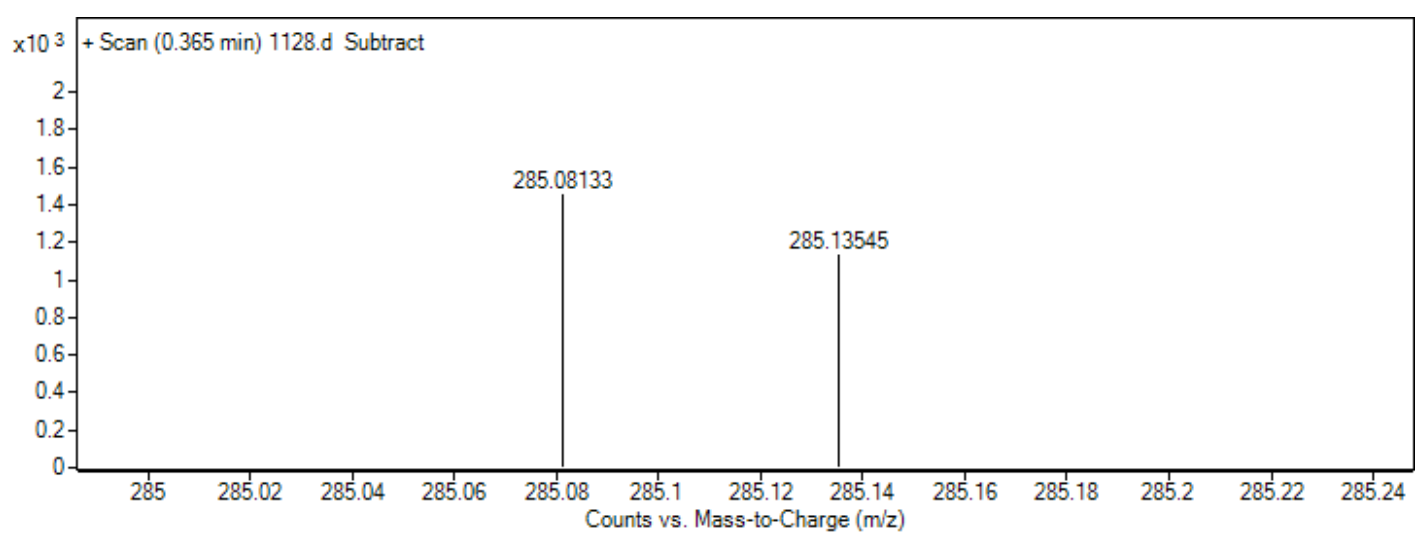<smiles>Cc1cc(O)c(C(C)C)cc1C(=O)C(=O)c1ccccc1</smiles>

3ad- ${ }^{18} \mathrm{O}$

HRMS (ESI): $\mathrm{m} / \mathrm{z}[\mathrm{M}+\mathrm{H}]^{+}$calcd for $\mathrm{C}_{18} \mathrm{H}_{19} \mathrm{O}_{2}{ }^{18} \mathrm{O}: 285.1371$; found: 285.1355 . 


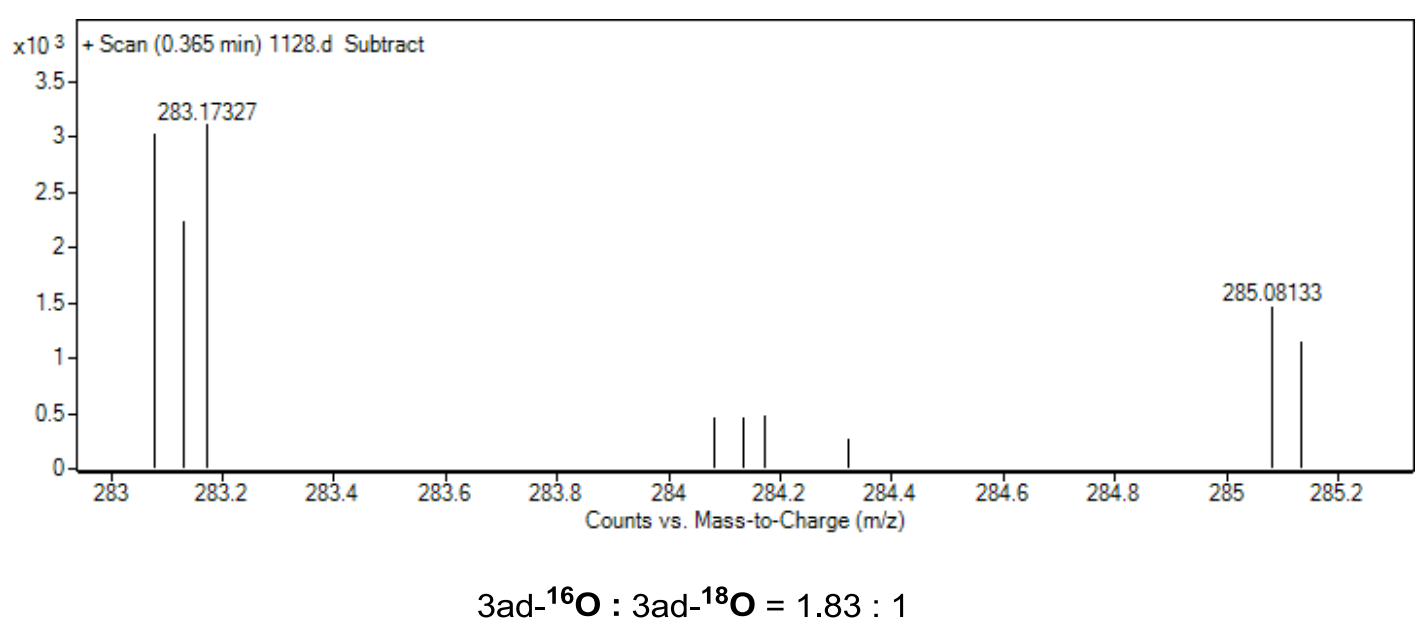

Notes: No 3ad- $-{ }^{18} \mathbf{O}$ was detected by HRMS, which determined that the oxygen atoms in product 3ad will not exchange with $\mathrm{H}_{2}{ }^{18} \mathrm{O}$.

\subsection{Reaction process monitored by ${ }^{1} \mathrm{H}$ and ${ }^{13} \mathrm{C}$ NMR spectroscopy.}

The reaction process was monitored by ${ }^{1} \mathrm{H}$ and ${ }^{13} \mathrm{C}$ NMR (using acetophenone- $\beta-{ }^{13} \mathrm{C}$ ) spectroscopy in DMSO- $d_{6}$. A rise-and fall-effect of the peaks at $\sim 5.7 \mathrm{ppm}$ for ${ }^{1} \mathrm{H}$ and $\sim 89.0 \mathrm{ppm}$ for ${ }^{13} \mathrm{C}$ was observed, which indicated that phenylglyoxal monohydrate was the reaction intermediate. This contrasts with our previous work ( (a) Gao, Q. H.; Fei, Z.; Zhu, Y. P.; Lian, M.; Jia, F. C.; Liu, M. C.; She, N. F.; Wu, A. X. Tetrahedron, 2013, 69, 22. (b) Zhu, Y. P.; Lian, M.; Jia, F. C.; Liu, M. C.; Yuan, J. J.; Gao, Q. H.; Wu, A. X. Chem. Commun., 2012, 48, 9086. (c) Zhu, Y. P.; Liu, M. C.; Cai, Q.; Jia, F. C.; Wu, A. X. Chem. Eur. J., 2013, 19, 10132.), in which 2-oxo-2-phenylacetaldehyde, with its characteristic peak at $\sim 9.55 \mathrm{ppm}$ for ${ }^{1} \mathrm{H}$, was the intermediate. The difference may be caused by the use of stoichiometric $\mathrm{H}_{2} \mathrm{O}$ via the hydroiodic acid solution herein, creating phenylglyoxal in its hydrous form.

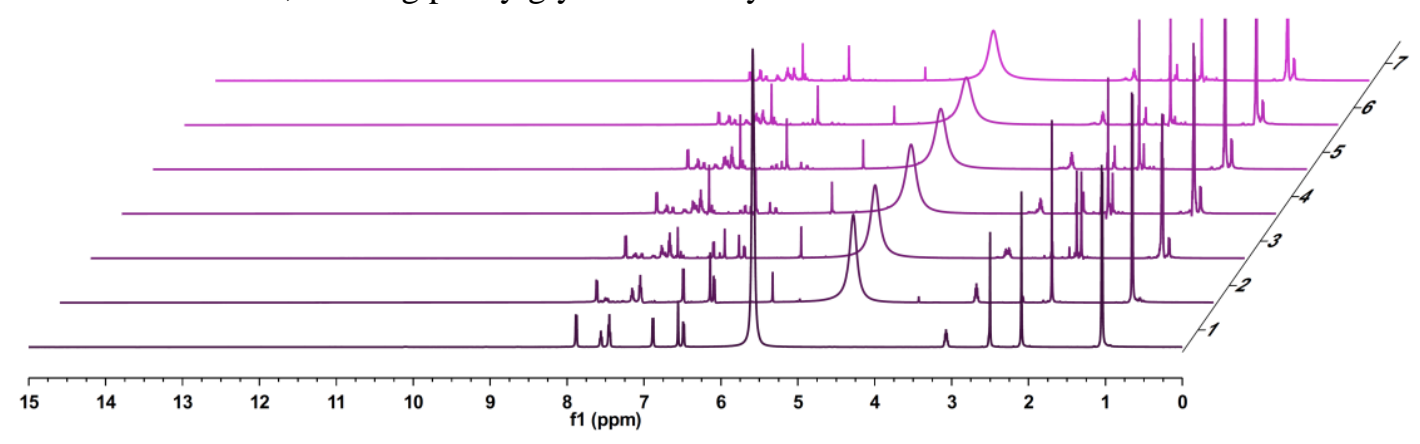

Fig. 3.2.1 The reaction process of $\mathbf{1 a}(0.1 \mathrm{mmol})$, $2 \mathbf{d}(0.2 \mathrm{mmol})$ in the presence of $\mathrm{HI}(0.05 \mathrm{mmol})$ and boric acid $(0.2 \mathrm{mmol})$ at $110{ }^{\circ} \mathrm{C}$, was monitored by ${ }^{1} \mathrm{H}$ NMR spectroscopy $(600 \mathrm{MHz}$, DMSO- $d 6,298+/-0.5 \mathrm{~K})$.

Remarks: The reaction was monitored by time: (1)-start; (2)-1 h; (3)-2 h; (4)-3 h; (5)-4h; (6)-5 h; (7)-6 $\mathrm{h}$. The board peaks at $\sim 5.6 \mathrm{ppm}$ and $\sim 4.3 \mathrm{ppm}$ were HI. A rise-and fall-effect of the peaks at $\sim 5.7 \mathrm{ppm}$ should be $-\mathrm{OH}$ of phenylglyoxal monohydrate (III). However, the peak at $\sim 9.55 \mathrm{ppm}$ which regard as $-\mathrm{CHO}$ of 2-oxo-2-phenylacetaldehyde (III') was not been observed during the process. 


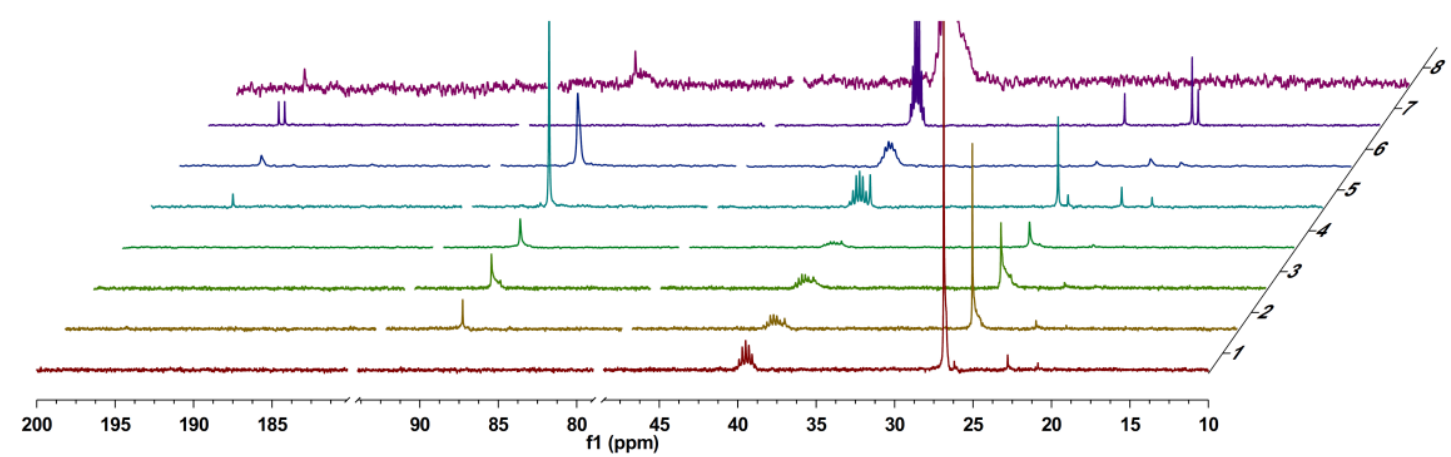

Fig. 3.2.2 The reaction process of $1 \mathbf{a}^{-13} \mathrm{C}$ (acetophenone- $\left.\beta-{ }^{13} \mathrm{C}\right)(0.1 \mathrm{mmol}), \mathbf{2 d}(0.2 \mathrm{mmol})$ in the presence of $\mathrm{HI}(0.05 \mathrm{mmol})$ and boric acid $(0.2 \mathrm{mmol})$ at $110{ }^{\circ} \mathrm{C}$, was monitored by ${ }^{13} \mathrm{C}$ NMR spectroscopy (100 MHz, DMSO- $d 6,298+/-0.5 \mathrm{~K}$ ).

Remarks: The reaction was monitored by time: (1)-start; (2)-1 h; (3)-2 h; (4)-3 h; (5)-4h; (6)-5 h; (7)-6 h. (8)-standard spectrum of phenylglyoxal monohydrate (III). The peaks at $\sim 89.0 \mathrm{ppm}$ should be -CH of phenylglyoxal monohydrate (III).

\subsection{The interaction of III and boric acid.}

We explored the coordination of phenylglyoxal monohydrate with boric acid. Using NMR monitoring (Fig. 3.3.1), we found that boric acid was able to abstract the hydroxy protons of III: peaks of $-\mathrm{OH}$ and $J$ coupling of $-\mathrm{CH}$ were lost. Which is considered to be the key activating step of III.( (a) Boronic Acids. Edited by Dennis G. Hall 2005 WILEY-VCH Verlag GmbH \& Co. KGaA, Weinheim ISBN 3-527-30991-8.; (b) Lee, D.; Williamson, C. L.; Chan, L.; Taylor, M. S.; J. Am. Chem. Soc. 2012, 134, 8260.; (c) Lee, D.; Newman, S. G.; Taylor, M. S.; Org. Lett.; 2010, 12, 256.; (d) Stephan, D. W.; Erker, G.; Angew. Chem. Int. Ed.; 2015, 54, 6400.) This coordination environment, which proved very sensitive to chemical shift changes in ${ }^{19} \mathrm{~F}$ NMR titration, (Parks, D. J.; Blackwell, J. M.; Piers, W. E.; J. Org. Chem., 2000, 65, 3090.) was also confirmed by the occurrence of the stoichiometric interaction of III and $\mathbf{D}$, according to the perturbation in the chemical shift (Fig. 3.3.2).

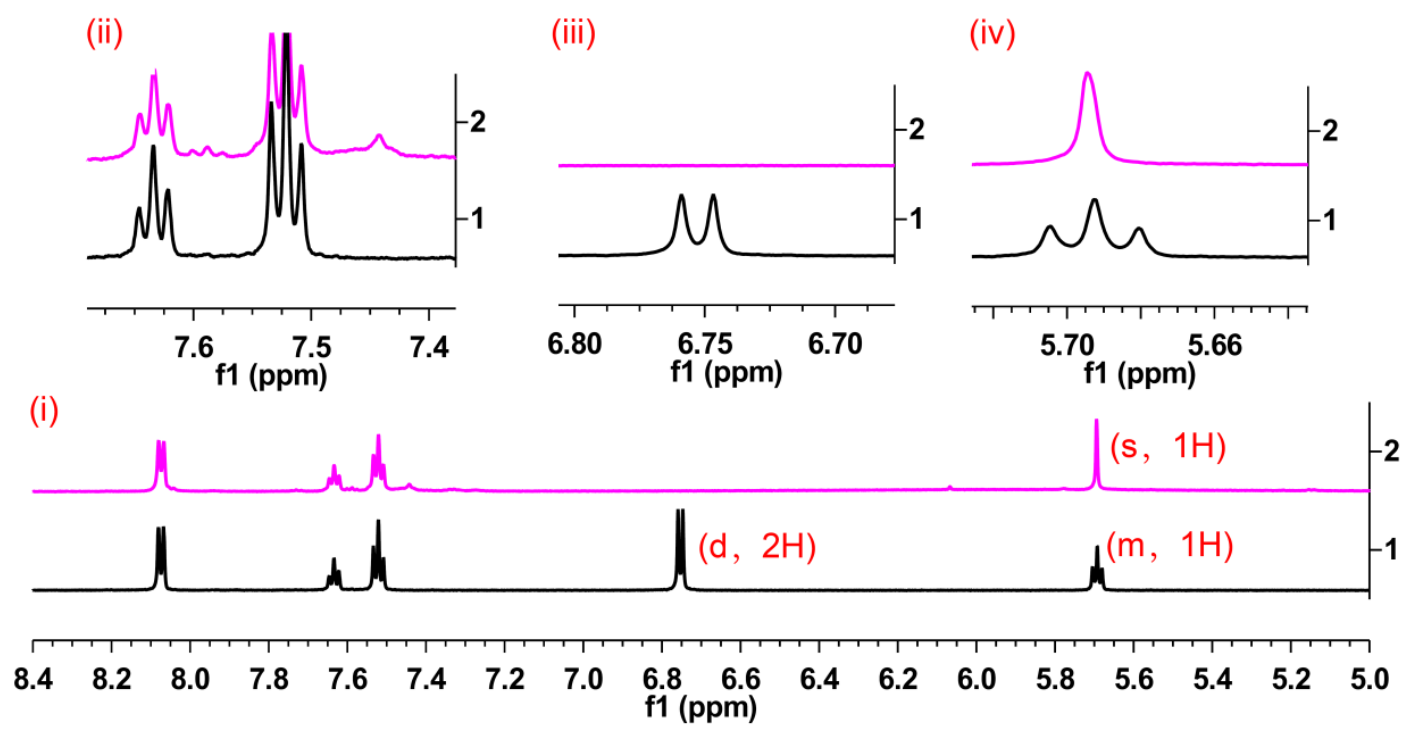

Fig. 3.3.1 ${ }^{1} \mathrm{H}$ NMR monitoring of the mixing of phenylglyoxal monohydrate (III) with boric acid, 
(1)-III; (2)-III+boric acid (1:2) was monitored by 'H NMR spectroscopy (600 MHz, DMSO-d6, $298+/-0.5 \mathrm{~K}$ ). for: (i) The full spectrum; (ii) Hydrogen on the aromatic ring; (iii) Peaks of $-\mathrm{OH}$ were lost; (iv) $J$ coupling of -CH were lost;

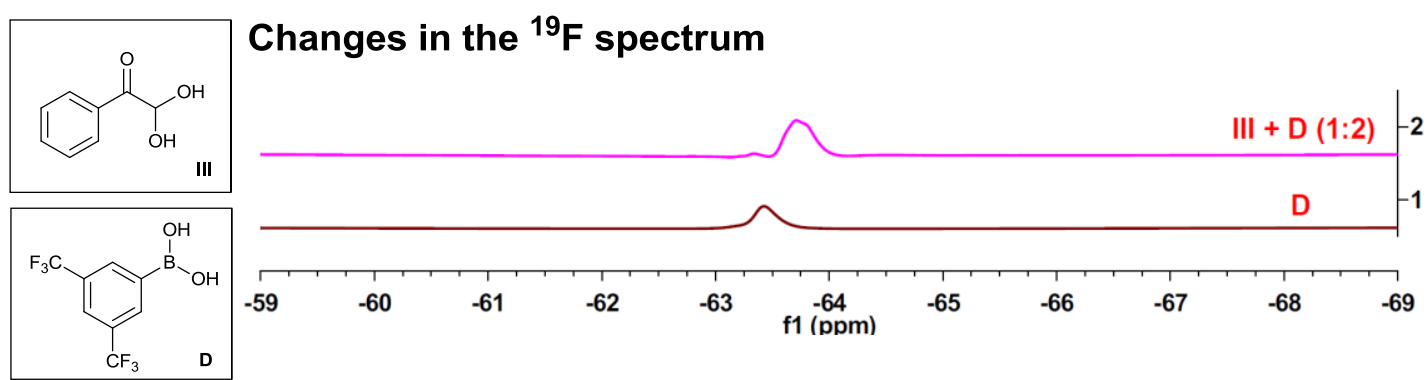

Fig. 3.3.2 ${ }^{19} \mathrm{~F}$ NMR titration of III and D. For (1)-D; (2)-D/III = 1:1; (3)-D/III = 1.5:1; (4)-D/III $=2.0: 1$ was monitored by ${ }^{19} \mathrm{~F}$ NMR spectroscopy (376 MHz, DMSO- $d 6,298+/-0.5 \mathrm{~K}$ ).

\subsection{The interaction of $2 \mathrm{~d}$ with boric acid monitored by ${ }^{1} \mathrm{H}$ NMR spectroscopy.}

Moreover, the interaction between boric acid and phenol was also investigated by NMR experiments (Fig. 3.4.1). The sharp peak of $\mathrm{O}-\mathrm{H}(\sim 9.0 \mathrm{ppm})$ became broader, indicating the formation of a certain amount of $[\mathrm{phenol}-\mathrm{O}]^{+}\left[\mathrm{HB}(\mathrm{OH})_{3}\right]^{-}$. The changes in the ${ }^{19} \mathrm{~F}$ NMR spectrum of the $\mathbf{D}$ and $\mathbf{2 d}$ mixture also support this conclusion: the peak of $\mathbf{D}$ shifted by $\sim 0.3 \mathrm{ppm}$ when adding 2d (Fig. 3.4.2). In this instance, boric acid activated III while passivating phenolic hydroxyl ( (a) Yu, D. G.; Shi, Z. J. Angew. Chem. Int. Ed., 2011, 50, 7097. (b) Xu, T. Y.; Sha, F.; Alper, H. J. Am. Chem. Soc., 2016, 138, 6629.) as a traceless protecting group, leading to para-selective coupling.

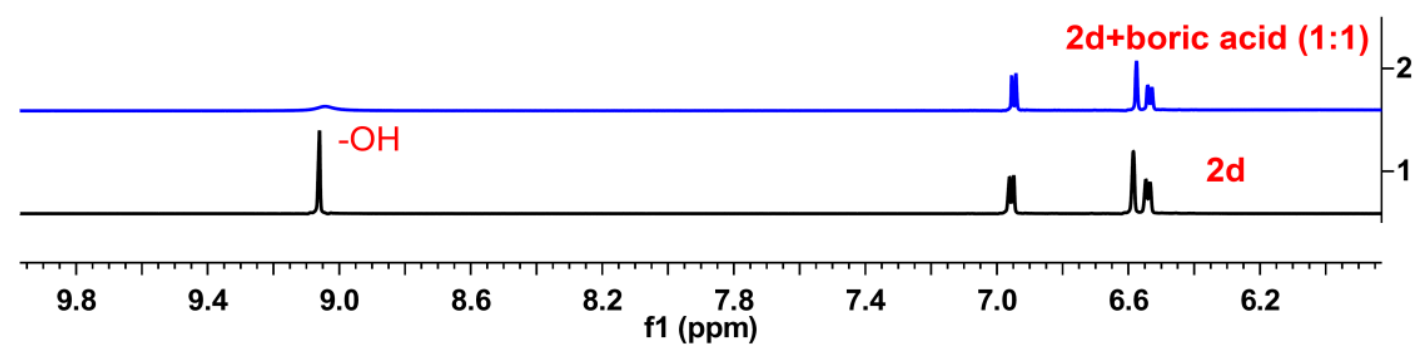

Fig. 3.4.1 ${ }^{1} \mathrm{H}$ NMR monitoring of the mixing of thymol (2d) with boric acid. (1)-2d; (2)-2d+boric acid (1:1) was monitored by 'H NMR spectroscopy (600 MHz, DMSO-d6, 298 +/-0.5 K).

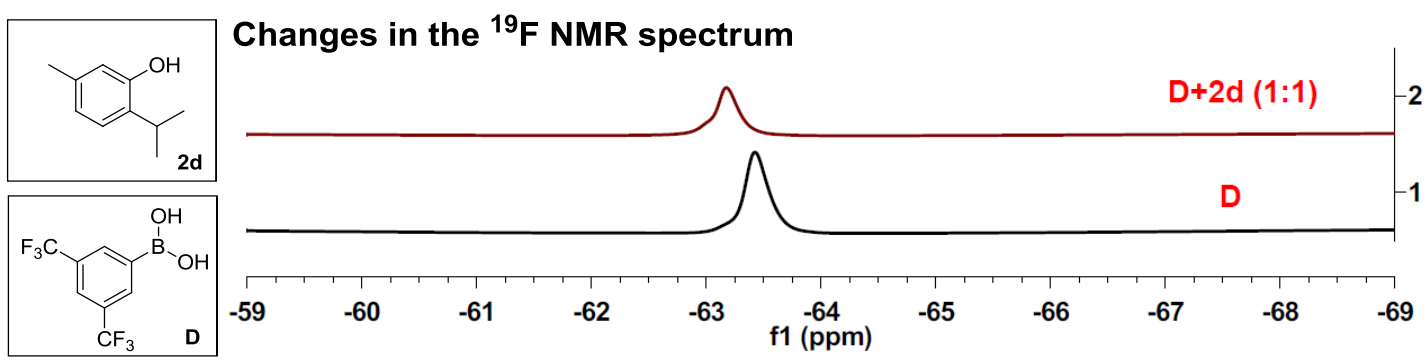

Fig. 3.4.2 ${ }^{19} \mathrm{~F}$ NMR titration of $\mathbf{2 d}$ with D. For (1)-D; (2)-D/2d = 1:1 was monitored by ${ }^{19} \mathrm{~F}$ NMR spectroscopy (376 MHz, DMSO- $d 6,298+/-0.5 \mathrm{~K})$.

\section{$3.5 \mathrm{pH}$ testing experiments process}

To further investigate the catalytic cycle of $\mathrm{HI}$, we treated $\mathrm{HI}$ in DMSO at $115^{\circ} \mathrm{C}$ and tested the 
$\mathrm{pH}$ both of this mixture and of our standard reaction. The reaction was carried on with HI (1.0 mmol) in $3.0 \mathrm{~mL}$ DMSO stirring at $115{ }^{\circ} \mathrm{C}$, taken $0.1 \mathrm{ml}$ reaction mixture each time and add to 20 $\mathrm{mL} \mathrm{H}_{2} \mathrm{O}$. Then $\mathrm{pH}$ value was measuring by $\mathrm{pH}$ meter. The $\mathrm{pH}$ of standard reaction was tested using a similar approach. Clearly similar downward trends were detected (Fig. 3.5). We believed that the reducing agent $\mathrm{HI}$ was oxidized by DMSO to afford $\mathrm{I}_{2}$ during the reaction process.

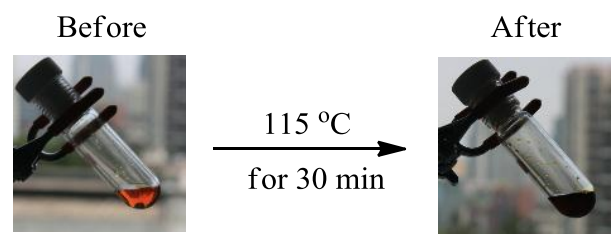

\begin{tabular}{cccc}
\hline conditons & $\mathbf{0 ~ h}(\mathbf{p H})$ & $\mathbf{1 h}(\mathbf{p H})$ & $\mathbf{2} \mathbf{h}(\mathbf{p H})$ \\
\hline DMSO, HI, $115^{\circ} \mathrm{C}$ & 2.73 & 3.17 & 3.61 \\
DMSO, HI, 1a, $\mathbf{2 a}$, broric acid, $115^{\circ} \mathrm{C}$ & 2.75 & 3.15 & 3.04 \\
\hline
\end{tabular}

Fig. 3.5 Investigation in the catalytic species: $\mathrm{pH}$ testaments of the reaction mixture

\subsection{Investigation in the terminal oxidant}

We considered that, when attacked by phenols, III might give a benzoin derivative intermediate. The terminal oxidative reaction was expected to form a carbonyl group: therefore, a series of terminal oxidants was also investigated. We used accessible benzoin as our model substrate. Four oxidant candidates, $\mathrm{I}_{2}$, DMSO, air (oxygen), and HI, were examined in our control experiments (Fig. 3.6). Only $I_{2}$ gave positive results, which means that $I_{2}$ is the appropriate terminal oxidant to promote the transformation. $\mathrm{HI}$ also transformed $\mathbf{7}$ into $\mathbf{8}$, but with poor effectiveness; we believe that this result was caused by the dissociation of $\mathrm{I}_{2}$ from the hydroiodic acid.

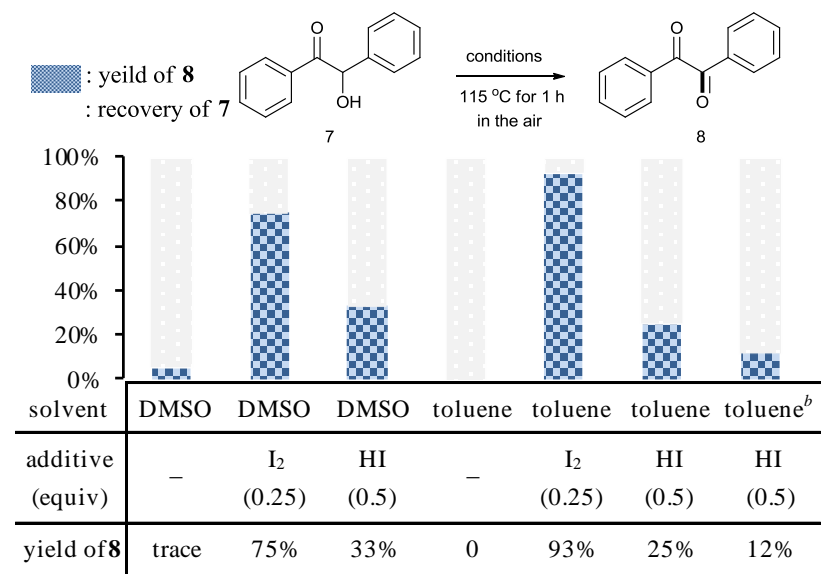

Fig. 3.6 ${ }^{\mathrm{a}}$ Reaction conditions: $7(0.5 \mathrm{mmol})$, addictive, solvent $(2.0 \mathrm{~mL}) .{ }^{\mathrm{b}} \mathrm{In}$ the Ar. ${ }^{\mathrm{c}}$ Isolated yields.

\subsection{Supplementary instruction of the selectivity of 3ak, 3kk, 3ek}

When using 3,4,5-trimethoxyphenol (2k) as substrate, the ortho-position of hydroxyl group is both a para-position and a ortho-position of electron donating methoxy groups which resulting a ortho-selective attack to III' in TS1. 


\section{Characterization data for target compound}

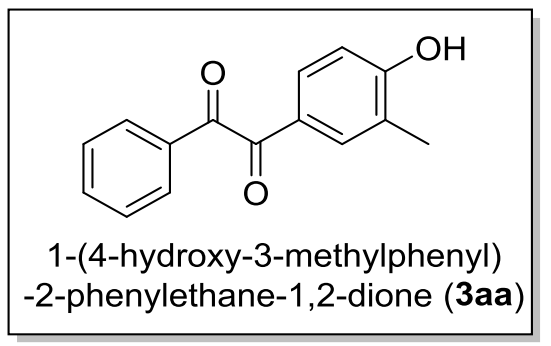

Yellow oil; IR (KBr) v $v_{\max }$ : 3368, 3065, 2926, 1672, 1647, 1580, 1503, 1451, 1369, 1324, 1292, 1241 , $1171,1127,1056,935,827,796,752,716,656,629,553,500,443 \mathrm{~cm}^{-1} ;{ }^{1} \mathrm{H}$ NMR $(600 \mathrm{MHz}$, DMSO- $\left.d_{6}\right) \delta 10.90(\mathrm{br}, 1 \mathrm{H}), 7.87(\mathrm{~d}, J=7.2 \mathrm{~Hz}, 2 \mathrm{H}), 7.75(\mathrm{t}, J=7.8 \mathrm{~Hz}, 1 \mathrm{H}), 7.69$ (s, 1H), 7.61-7.58 $(\mathrm{m}, J=7.4 \mathrm{~Hz}, 3 \mathrm{H}), 6.96(\mathrm{~d}, J=8.4 \mathrm{~Hz}, 1 \mathrm{H}), 2.16(\mathrm{~s}, 3 \mathrm{H}) .{ }^{13} \mathrm{C}$ NMR $\left(100 \mathrm{MHz}, \mathrm{DMSO}-d_{6}\right) \delta 195.50$, 193.35, 162.66, 135.21, 132.72, 132.36, 130.38, 129.49, 129.43, 125.59, 123.78, 115.18, 15.81. HRMS (ESI): $\mathrm{m} / \mathrm{z}[\mathrm{M}+\mathrm{Na}]^{+}$calcd for $\mathrm{C}_{15} \mathrm{H}_{12} \mathrm{NaO}_{3}$ : 263.0679; found: 263.0677 .

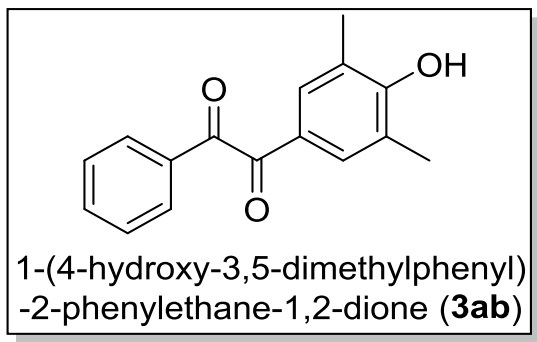

Yellow solid; $\mathrm{mp}=86-89{ }^{\circ} \mathrm{C}$; IR $(\mathrm{KBr}) v_{\max }$ : 3414, 2028, 1617, 1419, 1298, 1185, 1125, 1044, 1015, 951, 815, 761, 687, 620, $477 \mathrm{~cm}^{-1} ;{ }^{1} \mathrm{H}$ NMR (600 MHz, $\left.\mathrm{CDCl}_{3}\right) \delta 7.97(\mathrm{~d}, J=7.2 \mathrm{~Hz}, 2 \mathrm{H}), 7.67-7.62$ $(\mathrm{m}, 3 \mathrm{H}), 7.50(\mathrm{t}, J=7.8 \mathrm{~Hz}, 2 \mathrm{H}), 5.54(\mathrm{br}, 1 \mathrm{H}), 2.26(\mathrm{~s}, 6 \mathrm{H}) .{ }^{13} \mathrm{C} \mathrm{NMR}\left(150 \mathrm{MHz}, \mathrm{CDCl}_{3}\right) \delta 195.16$, $193.71,158.55,134.70,133.16,131.15,129.90,128.92,125.41,123.79,15.80$. HRMS (ESI): m/z $[\mathrm{M}+\mathrm{Na}]^{+}$calcd for $\mathrm{C}_{16} \mathrm{H}_{14} \mathrm{NaO}_{3}: 277.0835$; found: 277.0836 .

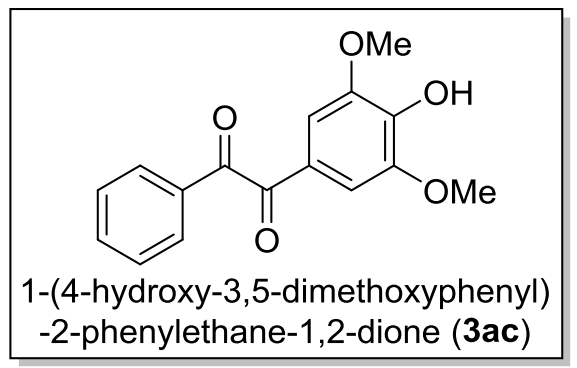

Yellow solid; $\mathrm{mp}=95-97{ }^{\circ} \mathrm{C}$; IR $(\mathrm{KBr}) v_{\max }: 3415,2925,1638,1417,1298,1182,1124,1044,1015$, 954, 814, 757, 688, 621, $600 \mathrm{~cm}^{-1} ;{ }^{1} \mathrm{H}$ NMR $\left(600 \mathrm{MHz}, \mathrm{CDCl}_{3}\right) \delta 7.98(\mathrm{~d}, J=7.8 \mathrm{~Hz}, 2 \mathrm{H}), 7.67(\mathrm{t}, J=$ $7.2 \mathrm{~Hz}, 1 \mathrm{H}), 7.52(\mathrm{t}, J=7.8 \mathrm{~Hz}, 2 \mathrm{H}), 7.26(\mathrm{~s}, 2 \mathrm{H}), 6.20(\mathrm{br}, 1 \mathrm{H}), 3.92(\mathrm{~s}, 6 \mathrm{H}) .{ }^{13} \mathrm{C} \mathrm{NMR}(150 \mathrm{MHz}$, $\left.\mathrm{CDCl}_{3}\right) \delta 194.44,192.98,147.11,141.46,134.80,133.13,129.94,128.95,124.41,107.02,56.50$. HRMS (ESI): m/z [M+Na] $]^{+}$calcd for $\mathrm{C}_{16} \mathrm{H}_{14} \mathrm{NaO}_{5}: 309.0733$; found: 309.0728 . 


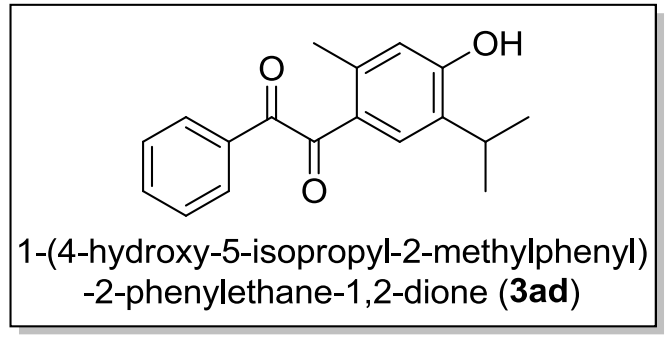

Yellow oil; IR (KBr) $v_{\max }$ : 3413, 2962, 2928, 2870, 1666, 1607, 1570, 1449, 1409, 1382, 1350, 1309, $1267,1218,1181,1155,1118,1044,1005,944,899,864,758,710,678,619,580,551 \mathrm{~cm}^{-1} ;{ }^{1} \mathrm{H}$ NMR $\left(600 \mathrm{MHz}\right.$, DMSO- $\left.d_{6}\right) \delta 10.76(\mathrm{br}, 1 \mathrm{H}), 7.90-7.82(\mathrm{~m}, 2 \mathrm{H}), 7.65(\mathrm{~d}, J=7.2 \mathrm{~Hz}, 1 \mathrm{H}), 7.52(\mathrm{t}, J=7.8 \mathrm{~Hz}$ 2H), $7.35(\mathrm{~s}, 1 \mathrm{H}), 6.81(\mathrm{~s}, 1 \mathrm{H}), 3.06(\mathrm{~m}, J=13.7,6.8 \mathrm{~Hz}, 1 \mathrm{H}), 2.50(\mathrm{~s}, 3 \mathrm{H}), 0.98(\mathrm{~d}, J=6.6 \mathrm{~Hz}, 6 \mathrm{H})$. ${ }^{13} \mathrm{C}$ NMR (150 MHz, DMSO- $d_{6}$ ) $\delta$ 195.52, 195.14, 160.57, 141.28, 134.99, 132.93, 132.34131 .99 , $129.45,122.44,119.07,118.88,26.27,21.97,21.59$. HRMS $(\mathrm{ESI}): \mathrm{m} / \mathrm{z}[\mathrm{M}+\mathrm{Na}]^{+}$calcd for $\mathrm{C}_{18} \mathrm{H}_{18} \mathrm{NaO}_{3}$ : 305.1148; found: 305.1145 .

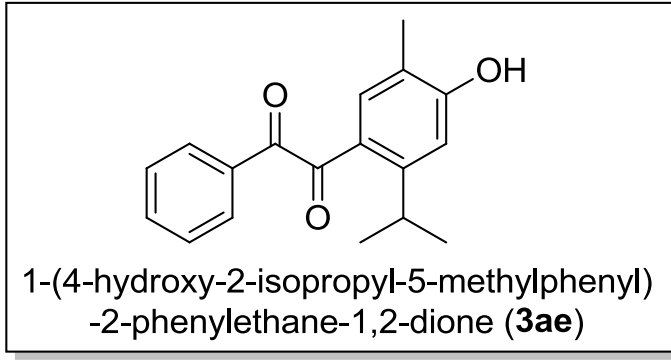

Yellow oil; IR (KBr) $v_{\max }: 3411,2963,2927,2868,1663,1576,1504,1451,1404,1315,1262,1215$, $1177,1149,1116,1068,1042,1010,892,861,798,768,724,679,630,570,533,472 \mathrm{~cm}^{-1} ;{ }^{1} \mathrm{H}$ NMR $\left(600 \mathrm{MHz}\right.$, DMSO- $\left.d_{6}\right) \delta 10.73(\mathrm{br}, 1 \mathrm{H}), 7.87(\mathrm{~d}, J=7.8 \mathrm{~Hz}, 2 \mathrm{H}), 7.76(\mathrm{t}, J=7.2 \mathrm{~Hz}, 1 \mathrm{H}), 7.61(\mathrm{t}, J=$ $7.8 \mathrm{~Hz}, 2 \mathrm{H}), 7.26(\mathrm{~s}, 1 \mathrm{H}), 7.02(\mathrm{~s}, 1 \mathrm{H}), 3.99(\mathrm{~m}, 1 \mathrm{H}), 2.03(\mathrm{~s}, 3 \mathrm{H}), 1.21(\mathrm{~d}, J=6.6 \mathrm{~Hz}, 6 \mathrm{H}) .{ }^{13} \mathrm{C} \mathrm{NMR}$ $\left(100 \mathrm{MHz}, \mathrm{DMSO}-d_{6}\right) \delta 204.93,204.38,189.88,171.28,162.15,146.44,144.69,142.56,139.22$, 139.16, 131.74, 130.94, 122.94, 38.41, 33.50, 25.25. HRMS (ESI): $\mathrm{m} / \mathrm{z}[\mathrm{M}+\mathrm{Na}]^{+}$calcd for $\mathrm{C}_{18} \mathrm{H}_{18} \mathrm{NaO}_{3}$ : 305.1148; found: 305.1147 .

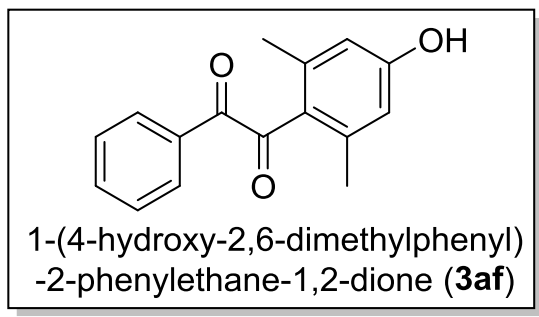

Yellow solid; IR (KBr) $v_{\max }$ : 3420, 2254, 2126, 1640, 1447, 1383, 1320, 1210, 1153, 1025, 999, 854, $827,768,721,626,533 \mathrm{~cm}^{-1} ; \mathrm{mp}=65-67{ }^{\circ} \mathrm{C} ;{ }^{1} \mathrm{H}$ NMR $\left(600 \mathrm{MHz}\right.$, DMSO- $\left.d_{6}\right) \delta 10.30(\mathrm{br}, 1 \mathrm{H}), 8.03(\mathrm{~d}$ $J=7.8 \mathrm{~Hz}, 2 \mathrm{H}), 7.71(\mathrm{t}, J=7.2 \mathrm{~Hz}, 1 \mathrm{H}), 7.58(\mathrm{t}, J=7.8 \mathrm{~Hz}, 2 \mathrm{H}), 6.60(\mathrm{~s}, 2 \mathrm{H}), 2.22(\mathrm{~s}, 6 \mathrm{H}) .{ }^{13} \mathrm{C}$ NMR $\left(150 \mathrm{MHz}\right.$, DMSO- $\left.d_{6}\right) \delta 196.21,192.70,160.72,141.36,132.01,130.17,125.81,116.63,116.10$, 115.54, 21.55. ${ }^{13} \mathrm{C}$ NMR (100 MHz, DMSO- $\left.d_{6}\right) \delta 196.22,192.68,160.69,141.32,134.72,132.02$, 130.14, 129.26, 125.83, 116.07, 21.51. HRMS (ESI): $\mathrm{m} / \mathrm{z}[\mathrm{M}+\mathrm{Na}]^{+}$calcd for $\mathrm{C}_{16} \mathrm{H}_{14} \mathrm{NaO}_{3}: 277.0835$; found: 277.0832 


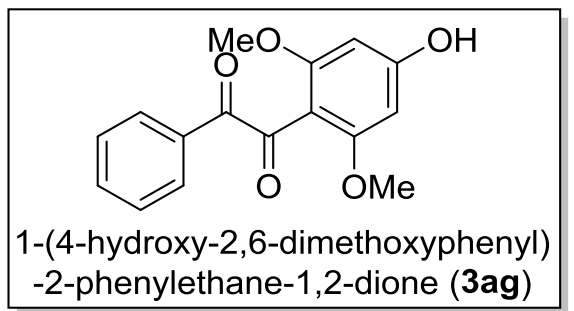

Pale yellow oil; IR (KBr) v $v_{\max }$ : 3421, 2923, 2850, 2255. 1638, 1580, 1450, 1348, 1214, 1165, 1134 , $1026,1000,867,826,766,729,684 \mathrm{~cm}^{-1} ;{ }^{1} \mathrm{H}$ NMR $\left(600 \mathrm{MHz}\right.$, DMSO- $\left.d_{6}\right) \delta 10.80(\mathrm{br}, 1 \mathrm{H}), 7.80(\mathrm{~d}, J$ $=7.8 \mathrm{~Hz}, 2 \mathrm{H}), 7.67(\mathrm{t}, J=7.2 \mathrm{~Hz}, 1 \mathrm{H}), 7.55(\mathrm{t}, J=7.8 \mathrm{~Hz}, 2 \mathrm{H}), 6.13(\mathrm{~s}, 2 \mathrm{H}), 3.57(\mathrm{~s}, 6 \mathrm{H}) .{ }^{13} \mathrm{C} \mathrm{NMR}$ $\left(100 \mathrm{MHz}\right.$, DMSO- $\left.d_{6}\right) \delta 191.11,190.62,165.02,162.80,133.20,132.67,128.77,128.55,105.04,92.41$, 55.68. HRMS (ESI): $\mathrm{m} / \mathrm{z}[\mathrm{M}+\mathrm{Na}]^{+}$calcd for $\mathrm{C}_{16} \mathrm{H}_{14} \mathrm{NaO}_{5}: 309.0733$; found: 309.0734 .

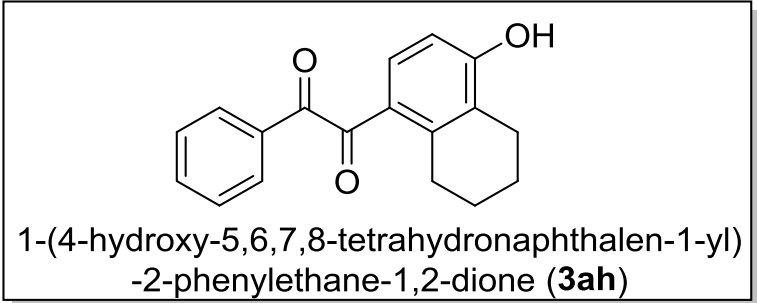

Yellow solid; $\mathrm{mp}=200-201{ }^{\circ} \mathrm{C}$; IR $(\mathrm{KBr}) v_{\max }: 3416,2928,2859,2366,1673,1595,1543,1486,1447$, $1297,1221,1181,1126,1043,1016,967,813,757,688,623,569 \mathrm{~cm}^{-1} ;{ }^{1} \mathrm{H} \mathrm{NMR}\left(600 \mathrm{MHz}, \mathrm{CDCl}_{3}\right) \delta$ $7.94(\mathrm{~d}, J=7.8 \mathrm{~Hz}, 2 \mathrm{H}), 7.63(\mathrm{t}, J=7.2 \mathrm{~Hz}, 1 \mathrm{H}), 7.49(\mathrm{t}, J=7.8 \mathrm{~Hz}, 2 \mathrm{H}), 7.32(\mathrm{~d}, J=8.4 \mathrm{~Hz}, 1 \mathrm{H})$, $6.56(\mathrm{~d}, J=9.0 \mathrm{~Hz}, 1 \mathrm{H}), 6.34(\mathrm{br}, 1 \mathrm{H}), 3.22(\mathrm{~m}, 2 \mathrm{H}), 2.62(\mathrm{~m}, 2 \mathrm{H}), 1.78(\mathrm{~m}, 4 \mathrm{H}) .{ }^{13} \mathrm{C}$ NMR $(150 \mathrm{MHz}$, $\left.\mathrm{CDCl}_{3}\right) \delta 195.81,159.05,144.18,134.66,134.03,133.21,129.99,128.93,125.81,123.99,111.67$, 28.75, 23.25, 22.36, 21.44. HRMS (ESI): $\mathrm{m} / \mathrm{z}[\mathrm{M}+\mathrm{Na}]^{+}$calcd for $\mathrm{C}_{18} \mathrm{H}_{16} \mathrm{NaO}_{3}: 303.0992$; found: 303.0996 .

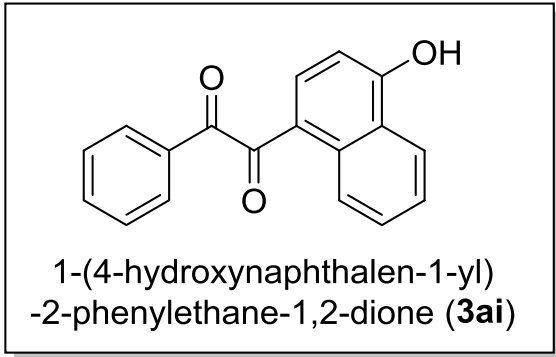

Brown solid; $\mathrm{mp}=129-131{ }^{\circ} \mathrm{C}$; IR (KBr) $v_{\max }: 3413,1673,1619,1561,1513,1450,1391,1354,1319$, 1290, 1253, 1218, 1180, 1114, 1070, 979, 854, 820, 768, 717, 684, 635, 575, $532 \mathrm{~cm}^{-1} ;{ }^{1} \mathrm{H}$ NMR (600 $\left.\mathrm{MHz}, \mathrm{CDCl}_{3}\right) \delta 9.42(\mathrm{~d}, J=8.4 \mathrm{~Hz}, 1 \mathrm{H}), 8.26(\mathrm{~d}, J=8.4 \mathrm{~Hz}, 1 \mathrm{H}), 8.02(\mathrm{~d}, J=7.8 \mathrm{~Hz}, 2 \mathrm{H}), 7.74(\mathrm{~m}$, 2H), $7.65(\mathrm{t}, J=7.2 \mathrm{~Hz}, 1 \mathrm{H}), 7.57(\mathrm{t}, J=7.8 \mathrm{~Hz}, 1 \mathrm{H}), 7.50(\mathrm{t}, J=7.8 \mathrm{~Hz}, 2 \mathrm{H}), 6.92(\mathrm{br}, 1 \mathrm{H}), 6.71(\mathrm{~d}, J$ $=8.4 \mathrm{~Hz}, 1 \mathrm{H}) .{ }^{13} \mathrm{C} \mathrm{NMR}\left(150 \mathrm{MHz}, \mathrm{CDCl}_{3}\right) \delta 195.76,195.42,158.60,138.19,134.76,133.42,133.03$, $130.25,130.10,128.98,126.44,125.96,124.64,122.43,121.12,107.51 . \mathrm{HRMS}(\mathrm{ESI}): \mathrm{m} / \mathrm{z}[\mathrm{M}+\mathrm{Na}]^{+}$ calcd for $\mathrm{C}_{18} \mathrm{H}_{12} \mathrm{NaO}_{3}: 299.0679$; found: 299.0676 . 


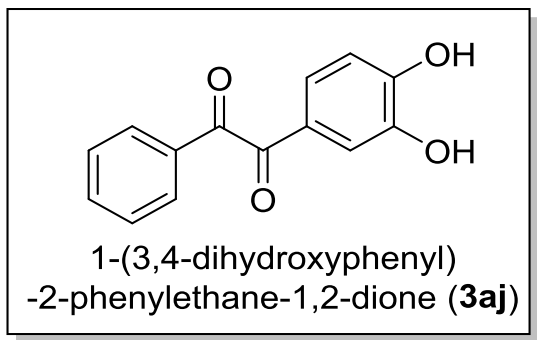

Yellow oil; IR (KBr) $v_{\max }: 3447,2924,1631,1386,1254,1103,590 \mathrm{~cm}^{-1} ;{ }^{1} \mathrm{H}$ NMR $(600 \mathrm{MHz}$, DMSO- $\left.d_{6}\right) \delta 10.06(\mathrm{br}, 2 \mathrm{H}), 7.89-7.85(\mathrm{~m}, 2 \mathrm{H}), 7.77(\mathrm{t}, J=7.2 \mathrm{~Hz}, 1 \mathrm{H}), 7.61(\mathrm{t}, J=7.8 \mathrm{~Hz}, 2 \mathrm{H}), 7.32$ $(\mathrm{m}, 1 \mathrm{H}), 7.21(\mathrm{~m}, 1 \mathrm{H}), 6.89(\mathrm{~d}, J=8.4 \mathrm{~Hz}, 1 \mathrm{H}) .{ }^{13} \mathrm{C}$ NMR $\left(150 \mathrm{MHz}\right.$, DMSO- $\left.d_{6}\right) \delta$ 195.72, 193.54, $153.40,146.22,135.33,132.80,129.55,124.35,124.25,116.12,115.44$. HRMS (ESI): $\mathrm{m} / \mathrm{z}[\mathrm{M}+\mathrm{Na}]^{+}$ calcd for $\mathrm{C}_{14} \mathrm{H}_{10} \mathrm{NaO}_{4}$ : 265.0471; found: 265.0476 .

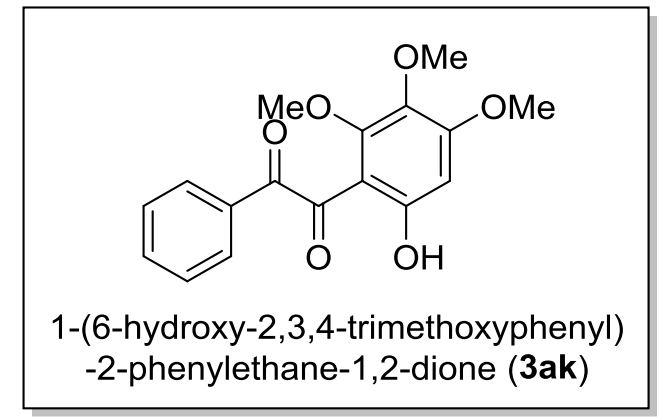

Yellow solid; $\mathrm{mp}=91-93{ }^{\circ} \mathrm{C}$; IR (KBr) $v_{\max }: 3333,3102,2926,2851,1715,1613,1483,1447,1421$, $1346,1314,1255,1201,1139,1100,990,911,826,800,763,707,593,537,483 \mathrm{~cm}^{-1} ;{ }^{1} \mathrm{H}$ NMR $(600$ $\left.\mathrm{MHz}, \mathrm{CDCl}_{3}\right) \delta 12.17(\mathrm{br}, 1 \mathrm{H}), 7.91(\mathrm{~d}, J=7.8 \mathrm{~Hz}, 2 \mathrm{H}), 7.62(\mathrm{t}, J=7.2 \mathrm{~Hz}, 1 \mathrm{H}), 7.51(\mathrm{t}, J=7.8 \mathrm{~Hz}$, $2 \mathrm{H}), 6.30(\mathrm{~s}, 1 \mathrm{H}), 3.92(\mathrm{~s}, 3 \mathrm{H}), 3.69(\mathrm{~s}, 3 \mathrm{H}), 3.58(\mathrm{~s}, 3 \mathrm{H}) .{ }^{13} \mathrm{C}$ NMR $\left(150 \mathrm{MHz}, \mathrm{CDCl}_{3}\right) \delta 197.71$, $190.38,162.81,162.32,154.16,133.93,133.78,132.75,129.12,128.78,106.29,95.80,60.90,60.38$, 56.34. HRMS (ESI): $\mathrm{m} / \mathrm{z}$ [M+Na] ${ }^{+}$calcd for $\mathrm{C}_{17} \mathrm{H}_{16} \mathrm{NaO}_{6}: 339.0839$; found: 339.0835 .

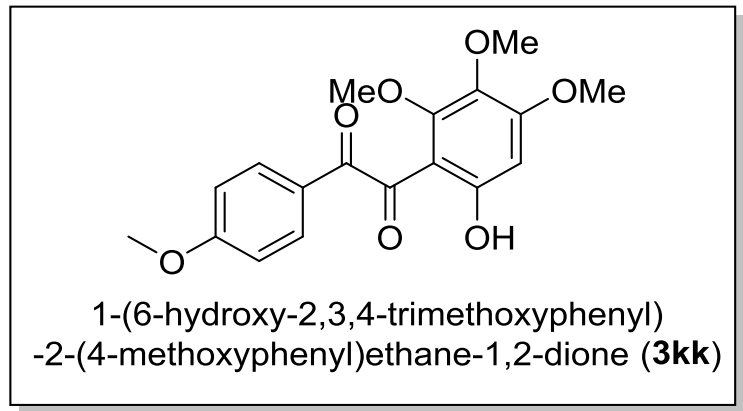

Yellow solid; $\mathrm{mp}=86-88{ }^{\circ} \mathrm{C}$; IR (KBr) $v_{\max }: 3449,2940,2844,1667,1601,1490,1451,1420,1352$, $1301,1251,1203,1168,1102,1055,1022,986,925,897,842,755,688,638,589,543 \mathrm{~cm}^{-1} ;{ }^{1} \mathrm{H}$ NMR $\left(600 \mathrm{MHz}, \mathrm{DMSO}-d_{6}\right) \delta 8.23(\mathrm{br}, 1 \mathrm{H}), 7.37(\mathrm{~d}, J=9.0 \mathrm{~Hz}, 2 \mathrm{H}), 6.93(\mathrm{~d}, J=8.4 \mathrm{~Hz}, 2 \mathrm{H}), 6.69(\mathrm{~s}, 1 \mathrm{H})$, 3.95 (s, 3H), 3.94 (s, 3H), 3.74 (s, 3H), 3.65 (s, 3H). ${ }^{13} \mathrm{C}$ NMR (150 MHz, DMSO- $d_{6}$ ) $\delta 193.40,168.08$, 163.24, 159.81, 150.87, 135.23, 129.07, 127.15, 113.71, 104.77, 103.35, 91.32, 61.55, 61.07, 56.90, 55.21. HRMS (ESI): $\mathrm{m} / \mathrm{z}[\mathrm{M}+\mathrm{Na}]^{+}$calcd for $\mathrm{C}_{18} \mathrm{H}_{18} \mathrm{NaO}_{7}$ : 369.0945; found: 369.0941 . 


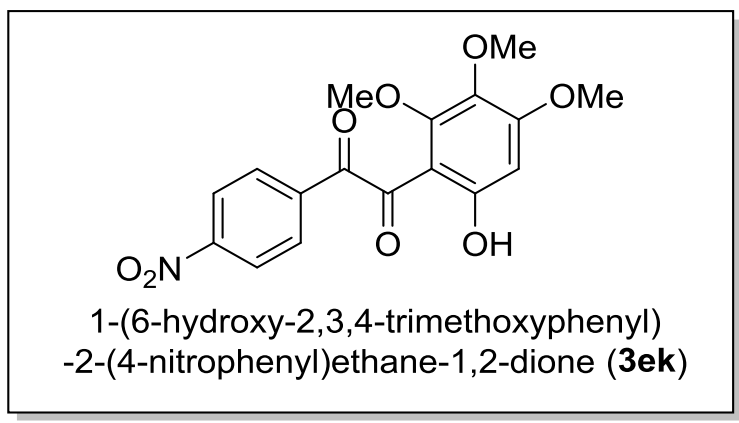

Yellow solid; $\mathrm{mp}=145-148{ }^{\circ} \mathrm{C}$; IR $(\mathrm{KBr}) v_{\max }: 3288,2948,2836,1698,1611,1525,1489,1425,1351$, 1302, 1263, 1197, 1170, 1133, 1062, 1001, 968, 908, 855, 788, 720, 687, $465 \mathrm{~cm}^{-1} ;{ }^{1} \mathrm{H}$ NMR (600 MHz, DMSO- $\left.d_{6}\right) \delta 8.70(\mathrm{br}, 1 \mathrm{H}), 8.26(\mathrm{~d}, J=8.4 \mathrm{~Hz}, 2 \mathrm{H}), 7.72(\mathrm{~d}, J=8.4 \mathrm{~Hz}, 2 \mathrm{H}), 6.78(\mathrm{~s}, 1 \mathrm{H}), 3.96$ $(\mathrm{s}, 6 \mathrm{H}), 3.67(\mathrm{~s}, 3 \mathrm{H}) .{ }^{13} \mathrm{C}$ NMR $\left(150 \mathrm{MHz}, \mathrm{DMSO}-d_{6}\right) \delta 192.10,168.09,163.64,150.90,147.97$, $143.93,135.55,127.30,123.69,103.85,103.01,91.54,61.59,61.08,57.03$. HRMS (ESI): $\mathrm{m} / \mathrm{z}$ $[\mathrm{M}+\mathrm{Na}]^{+}$calcd for $\mathrm{C}_{17} \mathrm{H}_{15} \mathrm{NNaO}_{8}: 384.0690$; found: 384.0693 .

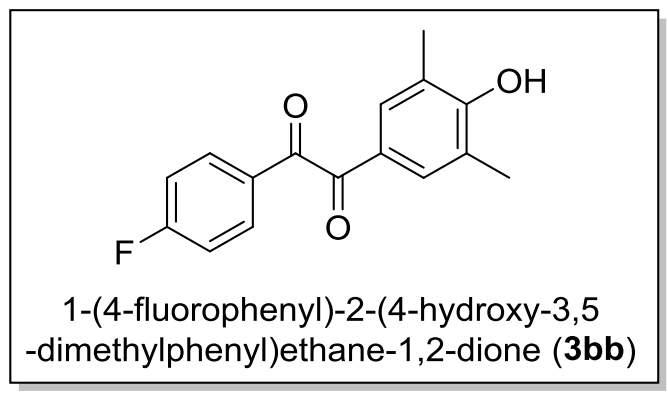

Yellow solid; $\mathrm{mp}=177-178{ }^{\circ} \mathrm{C}$; IR $(\mathrm{KBr}) v_{\max }: 3496,2920,1560,1594,1418,1385,1334,1277,1241$, 1193, 1151, 1031, 948, 903, 853, 753, 713, 611, 571, 503, $436 \mathrm{~cm}^{-1} ;{ }^{1} \mathrm{H} \mathrm{NMR}\left(600 \mathrm{MHz}, \mathrm{CDCl}_{3}\right) \delta$ $8.01(\mathrm{~m}, 2 \mathrm{H}), 7.62(\mathrm{~s}, 2 \mathrm{H}), 7.18(\mathrm{t}, J=8.4 \mathrm{~Hz}, 2 \mathrm{H}), 5.60(\mathrm{br}, 1 \mathrm{H}), 2.27(\mathrm{~s}, 6 \mathrm{H}) .{ }^{13} \mathrm{C} \mathrm{NMR}(100 \mathrm{MHz}$, DMSO- $\left.d_{6}\right) \delta 193.74,193.09,167.28,164.74,160.57,132.65,132.60,130.60,129.47,129.44,125.10$, 123.55, 116.79, 116.56, 16.48. HRMS (ESI): $\mathrm{m} / \mathrm{z}$ [M+Na] ${ }^{+}$calcd for $\mathrm{C}_{16} \mathrm{H}_{13} \mathrm{FNaO}_{3}$ : 295.0741; found: 295.0745 .

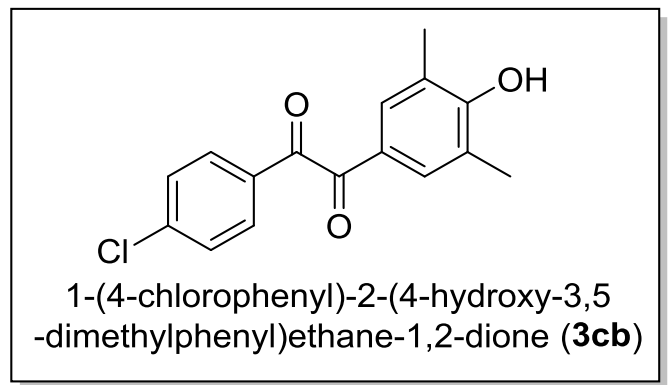

White solid; $\mathrm{mp}=216-217{ }^{\circ} \mathrm{C}$; IR $(\mathrm{KBr}) v_{\max }: 3432,2920,1675,1646,1584,1488,1424,1386,1330$, 1274, 1196, 1164, 1127, 1090, 1043, 1015, 950, 902, 853, 820, 763, 686, 622, 572, $496 \mathrm{~cm}^{-1}$; ${ }^{1} \mathrm{H}$ NMR $\left(600 \mathrm{MHz}\right.$, acetone- $\left.d_{6}\right) \delta 8.47(\mathrm{br}, 1 \mathrm{H}), 7.75(\mathrm{~d}, J=8.4 \mathrm{~Hz}, 2 \mathrm{H}), 7.45(\mathrm{~d}, J=8.4 \mathrm{~Hz}, 2 \mathrm{H}), 7.42(\mathrm{~s}, 2 \mathrm{H})$, $2.09(\mathrm{~s}, 6 \mathrm{H}) .{ }^{13} \mathrm{C}$ NMR $\left(100 \mathrm{MHz}\right.$, acetone- $\left.d_{6}\right) \delta 195.60,194.64,161.80,142.15,133.62,132.83,132.42$, 131.04, 126.49, 126.26, 17.27. HRMS (ESI): $\mathrm{m} / \mathrm{z}[\mathrm{M}+\mathrm{Na}]^{+}$calcd for $\mathrm{C}_{16} \mathrm{H}_{13} \mathrm{ClNaO}_{3}$ : 311.0445 ; found: 311.0443 . 


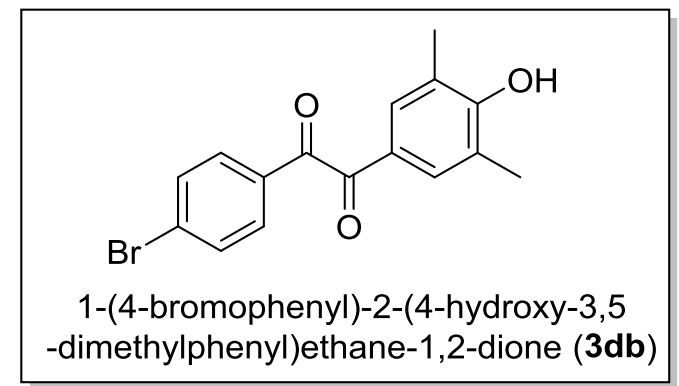

Yellow solid; $\mathrm{mp}=209-210{ }^{\circ} \mathrm{C}$; IR $(\mathrm{KBr}) v_{\max }: 3416,2925,1640,1420,1385,1302,1185,1152,1126$, 1044, 1015, 953, 816, 759, 687, 621, $570 \mathrm{~cm}^{-1}$; ${ }^{1} \mathrm{H}$ NMR (600 MHz, $\left.\mathrm{CDCl}_{3}\right) \delta 7.83(\mathrm{~d}, J=8.4 \mathrm{~Hz}, 2 \mathrm{H})$, $7.65(\mathrm{~d}, J=8.4 \mathrm{~Hz}, 2 \mathrm{H}), 7.62(\mathrm{~s}, 2 \mathrm{H}), 5.39(\mathrm{br}, 1 \mathrm{H}), 2.27(\mathrm{~s}, 6 \mathrm{H}) .{ }^{13} \mathrm{C} \mathrm{NMR}\left(150 \mathrm{MHz}, \mathrm{CDCl}_{3}\right) \delta$ $193.84,192.92,158.62,131.98,131.25,131.21,130.22,125.28,123.81,15.78$. HRMS (ESI): m/z $[\mathrm{M}+\mathrm{Na}]^{+}$calcd for $\mathrm{C}_{16} \mathrm{H}_{13} \mathrm{BrNaO}_{3}$ : 354.9940; found: 354.9941 .

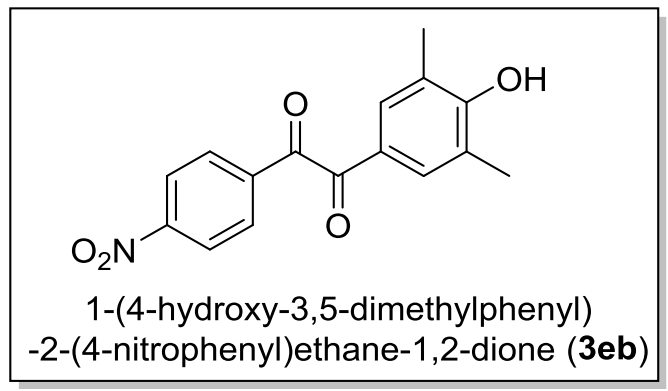

Yellow oil; IR (KBr) v $v_{\max }$ : 3543, 3451, 3102, 2923, 1678, 1647, 1598, 1525, 1489, 1384, 1347, 1260 , 1202, 1162, 1081, 1010, 947, 902, 866, 819, 782, 759, 735, 710, 688, 502, $452 \mathrm{~cm}^{-1}$; ${ }^{1} \mathrm{H}$ NMR (600 MHz, DMSO- $\left.d_{6}\right) \delta 9.86(\mathrm{br}, 1 \mathrm{H}), 8.39(\mathrm{~d}, J=7.8 \mathrm{~Hz}, 2 \mathrm{H}), 8.12(\mathrm{~d}, J=8.4 \mathrm{~Hz}, 2 \mathrm{H}), 7.58(\mathrm{~s}, 2 \mathrm{H}), 2.21$ $(\mathrm{s}, 6 \mathrm{H}) .{ }^{13} \mathrm{C}$ NMR $\left(150 \mathrm{MHz}, \mathrm{DMSO}-d_{6}\right) \delta 193.74,192.19,161.05,150.88,136.99,131.03,130.97$, 125.24, 124.49, 123.11, 16.52. HRMS (ESI): $\mathrm{m} / \mathrm{z}[\mathrm{M}+\mathrm{Na}]^{+}$calcd for $\mathrm{C}_{16} \mathrm{H}_{13} \mathrm{NNaO}_{5}$ : 322.0686 ; found: 322.0685 .

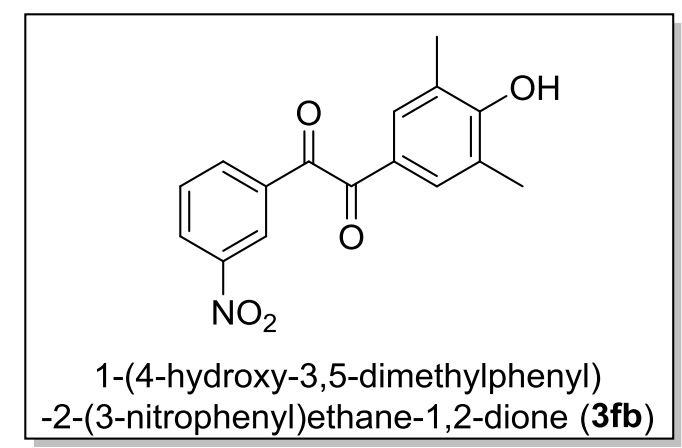

Yellow solid; $\mathrm{mp}=90-93{ }^{\circ} \mathrm{C}$; IR $(\mathrm{KBr}) v_{\max }: 3439,2923,2853,1631,1531,1473,1385,1349,1261$, 1199, 1144, 1019, 850, 763, $698 \mathrm{~cm}^{-1}$; ${ }^{1} \mathrm{H}$ NMR (600 MHz, DMSO- $\left.d_{6}\right) \delta 9.86(\mathrm{br}, 1 \mathrm{H}), 8.66-8.48(\mathrm{~m}$, $2 \mathrm{H}), 8.28(\mathrm{~d}, J=7.8 \mathrm{~Hz}, 1 \mathrm{H}), 7.89(\mathrm{t}, J=8.4 \mathrm{~Hz}, 1 \mathrm{H}), 7.60(\mathrm{~s}, 2 \mathrm{H}), 2.21(\mathrm{~s}, 6 \mathrm{H}) .{ }^{13} \mathrm{C} \mathrm{NMR}(150 \mathrm{MHz}$, 
DMSO- $\left.d_{6}\right) \delta 192.88,191.92,160.95,148.18,135.63,133.69,131.01,129.12,125.20,123.20,16.51$. HRMS (ESI): $\mathrm{m} / \mathrm{z}$ [M+Na] ${ }^{+}$calcd for $\mathrm{C}_{16} \mathrm{H}_{13} \mathrm{NNaO}_{5}$ : 322.0686; found: 322.0685 .

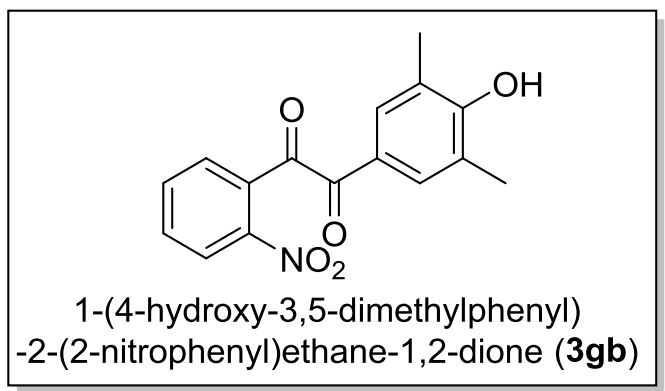

Yellow solid; $\mathrm{mp}=187-191{ }^{\circ} \mathrm{C}$; IR (KBr) $v_{\max }: 3420,2922,1709,1644,1596,1526,1488,1419,1384$, 1329, 1274, 1174, 1126, 1043, 1016, 943, 900, 861, 818, 762, 689, 647, $569 \mathrm{~cm}^{-1} ;{ }^{1} \mathrm{H}$ NMR (600 MHz, $\left.\mathrm{CD}_{3} \mathrm{CN}\right) \delta 8.15(\mathrm{~d}, J=7.8 \mathrm{~Hz}, 1 \mathrm{H}), 7.90(\mathrm{t}, J=7.8 \mathrm{~Hz}, 1 \mathrm{H}), 7.86-7.79(\mathrm{~m}, 3 \mathrm{H}), 7.77(\mathrm{~d}, J=7.8 \mathrm{~Hz}$, $1 \mathrm{H}), 2.28$ (s, 6H). ${ }^{13} \mathrm{C}$ NMR (150 MHz, $\left.\mathrm{CD}_{3} \mathrm{CN}\right) \delta 191.56,187.96,160.83,148.71,136.41,134.33$, 134.22, 132.98, 132.27, 125.75, 125.63, 17.06. HRMS (ESI): $\mathrm{m} / \mathrm{z}[\mathrm{M}+\mathrm{Na}]^{+}$calcd for $\mathrm{C}_{16} \mathrm{H}_{13} \mathrm{NNaO}_{5}$ : 322.0686; found: 322.0682 .

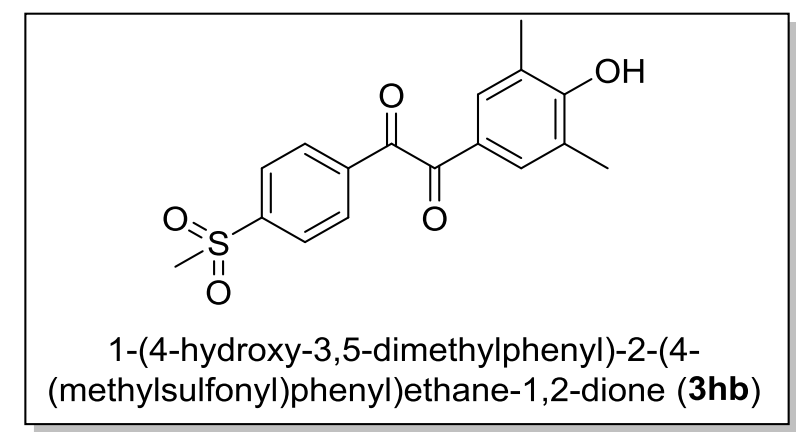

Yellow solid; $\mathrm{mp}=206-208{ }^{\circ} \mathrm{C}$; IR $(\mathrm{KBr}) v_{\max }: 3427,2921,1686,1652,1584,1489,1422,1324,1203$, $1160,1087,1035,948,865,823,758,684,573,530,496 \mathrm{~cm}^{-1} ;{ }^{1} \mathrm{H}$ NMR $\left(600 \mathrm{MHz}, \mathrm{CDCl}_{3}\right) \delta 8.16(\mathrm{~d}$, $J=8.4 \mathrm{~Hz}, 2 \mathrm{H}), 8.09(\mathrm{~d}, J=8.4 \mathrm{~Hz}, 2 \mathrm{H}), 7.64(\mathrm{~s}, 2 \mathrm{H}), 5.56(\mathrm{br}, 1 \mathrm{H}), 3.10(\mathrm{~s}, 3 \mathrm{H}), 2.29(\mathrm{~s}, 6 \mathrm{H}) .{ }^{13} \mathrm{C}$ NMR (150 MHz, $\left.\mathrm{CDCl}_{3}\right) \delta 193.03,192.10,159.01,145.19,137.11,131.32,130.70,127.98,124.86$, 124.03, 44.28, 15.83. HRMS (ESI): $\mathrm{m} / \mathrm{z}$ [M+Na] $]^{+}$calcd for $\mathrm{C}_{17} \mathrm{H}_{16} \mathrm{NaO}_{5} \mathrm{~S}: 355.0611$; found: 355.0610 .

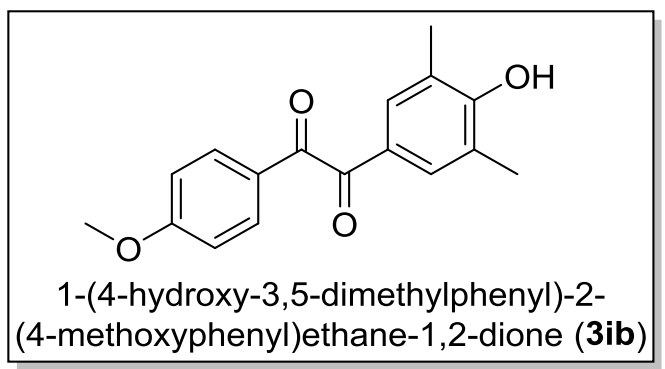

Pale yellow solid; $\mathrm{mp}=119-121{ }^{\circ} \mathrm{C}$; IR $(\mathrm{KBr}) v_{\max }: 3422,2923,2848,1683,1602,1513,1424,1386$, 1301, 1260, 1180, 1126, 1043, 1018, 948, 845, 817, 769, 689, 614, 571, $505 \mathrm{~cm}^{-1} ;{ }^{1} \mathrm{H}$ NMR (600 MHz, DMSO- $\left.d_{6}\right) \delta 9.72(\mathrm{br}, 1 \mathrm{H}), 7.82(\mathrm{~d}, J=8.4 \mathrm{~Hz}, 2 \mathrm{H}), 7.50(\mathrm{~s}, 2 \mathrm{H}), 7.11(\mathrm{~d}, J=8.4 \mathrm{~Hz}, 2 \mathrm{H}), 3.85(\mathrm{~s}, 3 \mathrm{H})$, $2.20(\mathrm{~s}, 6 \mathrm{H}) .{ }^{13} \mathrm{C}$ NMR $\left(150 \mathrm{MHz}\right.$, DMSO- $\left.d_{6}\right) \delta 193.93,193.87,164.64,160.33,131.97,130.47$, 
125.63, 125.02, 123.91, 114.78, 55.83, 16.54. HRMS (ESI): $\mathrm{m} / \mathrm{z}[\mathrm{M}+\mathrm{Na}]^{+}$calcd for $\mathrm{C}_{17} \mathrm{H}_{16} \mathrm{NaO}_{4}$ : 307.0941; found: 307.0942 .

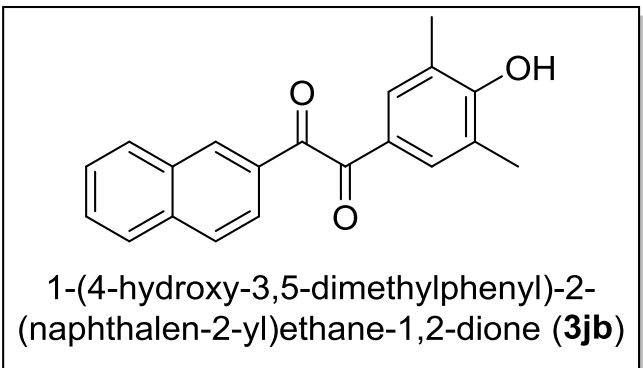

Yellow solid; $\mathrm{mp}=201-203{ }^{\circ} \mathrm{C}$; IR $(\mathrm{KBr}) v_{\max }: 3396,3052,2919,1658,1584,1485,1422,1389,1331$, 1271, 1203, 1160, 1122, 961, 908, 869, 807, 760, 703, 572, 500, $473 \mathrm{~cm}^{-1} ;{ }^{1} \mathrm{H}$ NMR (400 MHz, DMSO- $\left.d_{6}\right) \delta 8.44(\mathrm{~s}, 1 \mathrm{H}), 8.15-8.06(\mathrm{~m}, 2 \mathrm{H}), 7.99-7.95(\mathrm{~m}, 2 \mathrm{H}), 7.69-7.67(\mathrm{~m}, 1 \mathrm{H}), 7.64-7.47$ (m, 3H), 2.21 (s, 6H). ${ }^{13} \mathrm{C}$ NMR (100 MHz, DMSO- $\left.d_{6}\right) \delta 195.00,192.98,160.18,135.54,132.78$, 132.50, 131.70, 130.38, 129.81, 129.74, 128.99, 127.63, 127.15, 124.83, 123.54, 122.99, 16.55. HRMS (ESI): $\mathrm{m} / \mathrm{z}[\mathrm{M}+\mathrm{Na}]^{+}$calcd for $\mathrm{C}_{20} \mathrm{H}_{16} \mathrm{NaO}_{3}$ : 327.0992; found: 327.0995 .

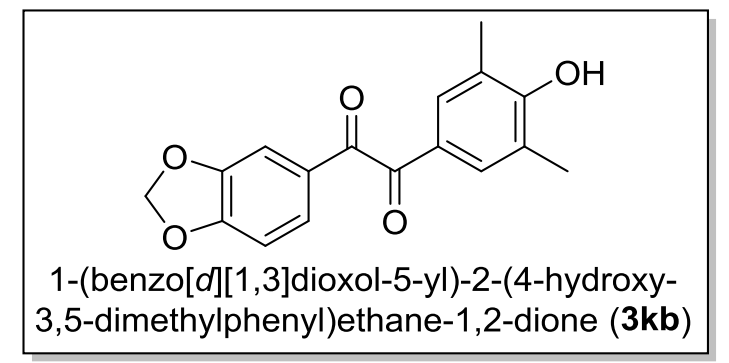

Yellow solid; mp = 174-176 ${ }^{\circ} \mathrm{C}$; IR $(\mathrm{KBr}) v_{\max }: 3415,3236,2029,1617,1492,1417,1385,1184,1123$, 1042, 966, 884, 815, 763, 620, $476 \mathrm{~cm}^{-1} ;{ }^{1} \mathrm{H}$ NMR (600 MHz, $\left.\mathrm{CDCl}_{3}\right) \delta 7.59$ (s, 2H), 7.47 (s, 2H), 6.85 $(\mathrm{d}, J=8.4 \mathrm{~Hz}, 1 \mathrm{H}), 6.08(\mathrm{~s}, 2 \mathrm{H}), 5.78(\mathrm{br}, 1 \mathrm{H}), 2.25(\mathrm{~s}, 6 \mathrm{H}) .{ }^{13} \mathrm{C} \mathrm{NMR}\left(150 \mathrm{MHz}, \mathrm{CDCl}_{3}\right) \delta 193.78$, 193.55, 158.59, 153.30, 148.50, 131.08, 127.98, 127.88, 125.37, 123.85, 108.31, 102.16, 15.81. HRMS (ESI): $\mathrm{m} / \mathrm{z}[\mathrm{M}+\mathrm{Na}]^{+}$calcd for $\mathrm{C}_{17} \mathrm{H}_{14} \mathrm{NaO}_{5}: 321.0733$; found: 321.0735 .

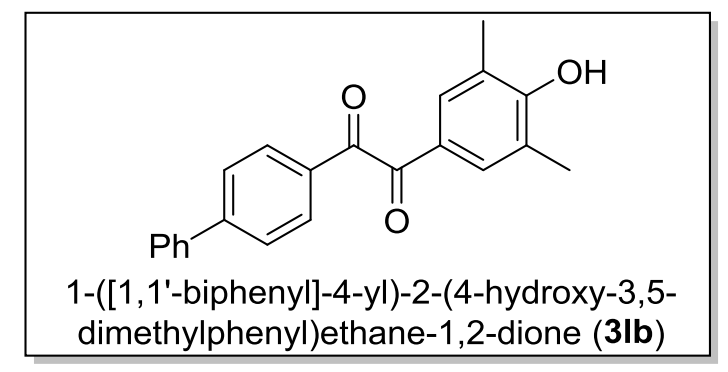

Yellow solid; $\mathrm{mp}=35-37{ }^{\circ} \mathrm{C}$; IR (KBr) $v_{\max }: 3415,2028,1638,1488,1383,1326,1188,1157,1125$, 1025, 949, 820, 764, 687, 620, $476 \mathrm{~cm}^{-1} ;{ }^{1} \mathrm{H}$ NMR $\left(600 \mathrm{MHz}\right.$, DMSO- $\left.d_{6}\right) \delta 9.77(\mathrm{br}, 1 \mathrm{H}), 7.94(\mathrm{~d}, J=$ $7.8 \mathrm{~Hz}, 2 \mathrm{H}), 7.90(\mathrm{~d}, J=7.8 \mathrm{~Hz}, 2 \mathrm{H}), 7.75(\mathrm{~d}, J=7.2 \mathrm{~Hz}, 2 \mathrm{H}), 7.56(\mathrm{~s}, 2 \mathrm{H}), 7.51(\mathrm{~m}, 2 \mathrm{H}), 7.45(\mathrm{~m}, 1 \mathrm{H})$, 2.21 (s, 6H). ${ }^{13} \mathrm{C}$ NMR $\left(150 \mathrm{MHz}\right.$, DMSO- $\left.d_{6}\right) \delta 194.95,193.54,160.55,146.44,138.53,131.45$, $130.58,130.17,129.19,128.84,127.60,127.18,125.13,123.70,16.53$. HRMS (ESI): $\mathrm{m} / \mathrm{z}[\mathrm{M}+\mathrm{Na}]^{+}$ calcd for $\mathrm{C}_{22} \mathrm{H}_{18} \mathrm{NaO}_{3}$ : 353.1148; found: 353.1145 . 


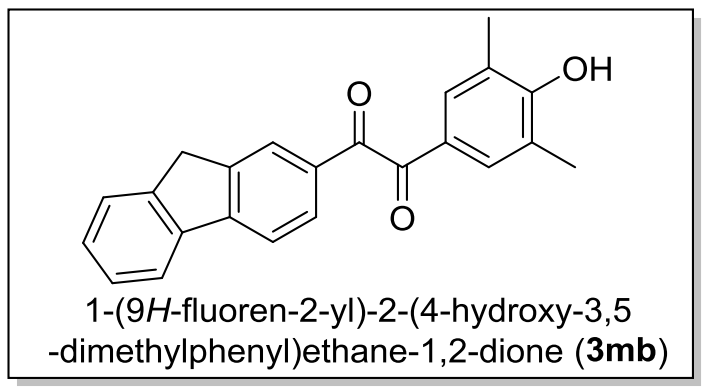

Yellow solid; $\mathrm{mp}=214-217{ }^{\circ} \mathrm{C}$; IR $(\mathrm{KBr}) v_{\max }: 3419,2962,2924,2365,1726,1639,1411,1303,1261$, 1183, 1150, 1123, 1043, 1016, 812, 756, 688, $670 \mathrm{~cm}^{-1} ;{ }^{1} \mathrm{H}$ NMR (600 MHz, DMSO-d $) \delta 9.75$ (br, $1 \mathrm{H}), 8.11(\mathrm{~d}, J=7.8 \mathrm{~Hz}, 1 \mathrm{H}), 8.06(\mathrm{~s}, 1 \mathrm{H}), 8.04(\mathrm{~d}, J=6.0 \mathrm{~Hz}, 1 \mathrm{H}), 7.91(\mathrm{~d}, J=7.8 \mathrm{~Hz}, 1 \mathrm{H}), 7.65$ (m, 1H), 7.55 (s, 2H), 7.44 (s, 2H), 4.03 (s, 2H), 2.21 (s, 6H). ${ }^{13} \mathrm{C}$ NMR (150 MHz, DMSO-d $) \delta 195.25$, $193.82,160.45,147.66,144.91,143.86,139.52,131.00,130.63,130.52,128.85,127.20,126.14$, 125.52, 125.09, 123.83, 121.56, 120.69, 36.48, 16.54. HRMS (ESI): m/z $[\mathrm{M}+\mathrm{Na}]^{+}$calcd for $\mathrm{C}_{23} \mathrm{H}_{18} \mathrm{NaO}_{3}$ : 365.1148; found: 365.1155 .

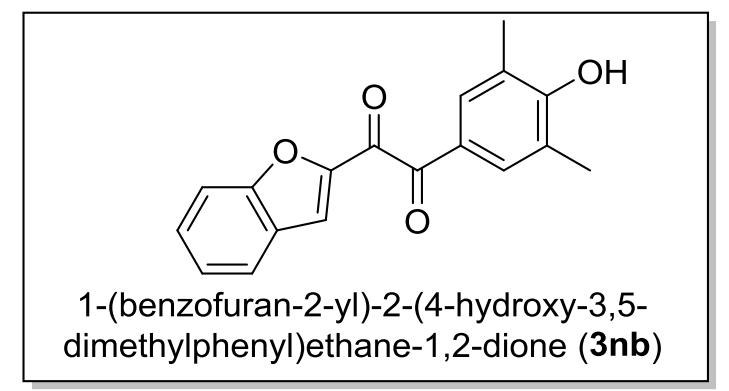

Yellow oil; IR (KBr) v $v_{\max }$ : 3480, 2920, 1656, 1593, 1547, 1490, 1382, 1326, 1301, 1272, 1189, 1132 , 1030, 967, 914, 889, 826, 736, 708, 564, $477 \mathrm{~cm}^{-1} ;{ }^{1} \mathrm{H}$ NMR (600 MHz, DMSO-d $) \delta 9.81$ (br, 1H), 7.92 (s, 1H), $7.86(\mathrm{~d}, J=7.8 \mathrm{~Hz}, 1 \mathrm{H}), 7.78(\mathrm{~d}, J=8.4 \mathrm{~Hz}, 1 \mathrm{H}), 7.65-7.60(\mathrm{~m}, 3 \mathrm{H}), 7.41(\mathrm{t}, J=7.2 \mathrm{~Hz}$, $1 \mathrm{H}), 2.21(\mathrm{~s}, 6 \mathrm{H}) .{ }^{13} \mathrm{C}$ NMR $\left(150 \mathrm{MHz}, \mathrm{DMSO}-d_{6}\right) \delta 190.72,184.27,160.73,155.84,149.25,130.99$, $129.98,126.60,125.10,124.60,124.46,123.10,119.67,112.56,16.56$. HRMS (ESI): m/z [M+Na] ${ }^{+}$ calcd for $\mathrm{C}_{18} \mathrm{H}_{14} \mathrm{NaO}_{4}$ : 317.0784; found: 317.0783 .

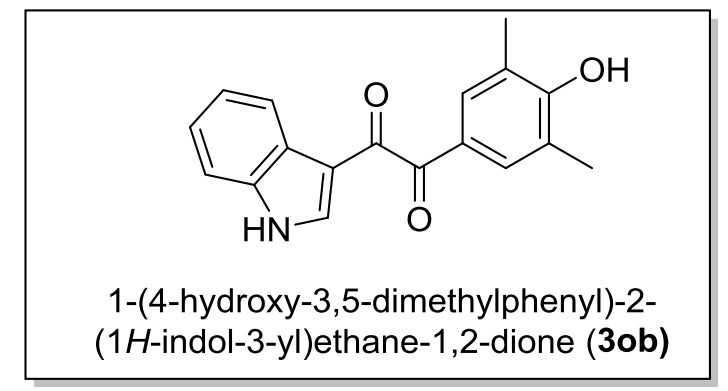

Black solid; $\mathrm{mp}=99-102{ }^{\circ} \mathrm{C}$; IR (KBr) $v_{\max }: 3414,2924,1734,1620,1421,1385,1319,1185,1151$, $1125,1043,1015,949,815,752,688,622,570 \mathrm{~cm}^{-1}$; ${ }^{1} \mathrm{H}$ NMR (600 MHz, DMSO-d $) \delta 12.33(\mathrm{br}, 1 \mathrm{H})$, 
$9.57(\mathrm{~s}, 1 \mathrm{H}), 8.20(\mathrm{~m}, 1 \mathrm{H}), 8.06(\mathrm{~s}, 1 \mathrm{H}), 7.57(\mathrm{~s}, 2 \mathrm{H}), 7.54(\mathrm{~d}, J=7.2 \mathrm{~Hz}, 1 \mathrm{H}), 7.29(\mathrm{~m}, 2 \mathrm{H}), 2.20(\mathrm{~s}$, $6 \mathrm{H}) .{ }^{13} \mathrm{C}$ NMR (150 MHz, DMSO- $\left.d_{6}\right) \delta 193.08,189.68,159.71,137.43,136.91,130.69,125.03$, 124.64, 124.25, 123.70, 122.67, 121.19, 112.85, 112.71, 16.60. HRMS (ESI): $\mathrm{m} / \mathrm{z}[\mathrm{M}+\mathrm{Na}]^{+}$calcd for $\mathrm{C}_{18} \mathrm{H}_{15} \mathrm{NNaO}_{3}$ : 316.0944; found: 316.0943 .

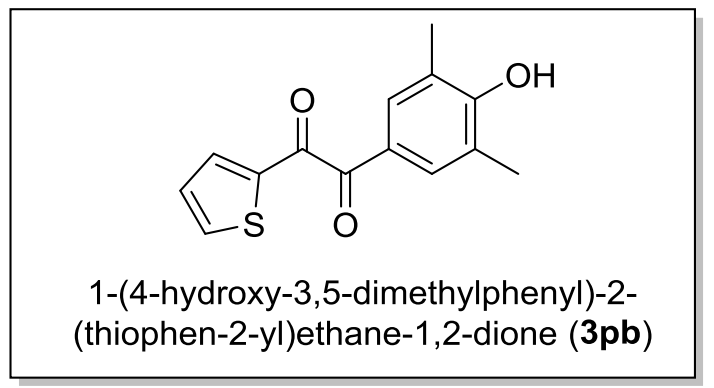

Yellow solid; $m p=182-185{ }^{\circ} \mathrm{C}$; IR $(\mathrm{KBr}) v_{\max }: 3414,1639,1409,1385,1300,1184,1151,1124,1044$, $1015,816,759,687,621,571,476 \mathrm{~cm}^{-1} ;{ }^{1} \mathrm{H}$ NMR $\left(600 \mathrm{MHz}\right.$, DMSO- $\left.d_{6}\right) \delta 9.73(\mathrm{br}, 1 \mathrm{H}), 8.27(\mathrm{~s}, 1 \mathrm{H})$, $7.76(\mathrm{~s}, 1 \mathrm{H}), 7.56(\mathrm{~s}, 2 \mathrm{H}), 7.30(\mathrm{~s}, 1 \mathrm{H}), 2.21(\mathrm{~s}, 6 \mathrm{H}) .{ }^{13} \mathrm{C}$ NMR (150 MHz, DMSO- $\left.d_{6}\right) \delta$ 191.30, 187.15, $160.56,139.35,138.26,137.32,130.85,129.60,125.02,123.18,16.56$. HRMS (ESI): $\mathrm{m} / \mathrm{z}[\mathrm{M}+\mathrm{Na}]^{+}$ calcd for $\mathrm{C}_{14} \mathrm{H}_{12} \mathrm{NaO}_{3} \mathrm{~S}: 283.0399$; found: 283.0394 .

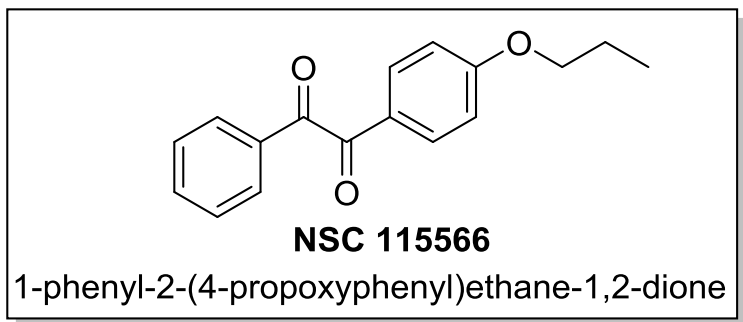

White solid; $\mathrm{mp}=101-103{ }^{\circ} \mathrm{C}$; IR (KBr) $v_{\max }: 3445,2965,1678,1657,1631,1598,1572,1509,1451$, 1425, 1396, 1317, 1291, 1261, 1220, 1171, 1063, 960, 885, 846, 797, 768, 714, 689, 647, 619, 520 $\mathrm{cm}^{-1} ;{ }^{1} \mathrm{H}$ NMR $\left(600 \mathrm{MHz}, \mathrm{CDCl}_{3}\right) \delta 7.97(\mathrm{~d}, J=7.2 \mathrm{~Hz}, 2 \mathrm{H}), 7.93(\mathrm{~d}, J=9.0 \mathrm{~Hz}, 2 \mathrm{H}), 7.64(\mathrm{t}, J=7.2$ $\mathrm{Hz}, 1 \mathrm{H}), 7.50(\mathrm{t}, J=7.8 \mathrm{~Hz}, 2 \mathrm{H}), 6.96(\mathrm{~d}, J=9.0 \mathrm{~Hz}, 2 \mathrm{H}), 4.00(\mathrm{t}, J=6.6 \mathrm{~Hz}, 2 \mathrm{H}), 1.83(\mathrm{~m}, 7.0 \mathrm{~Hz}$, 2H), $1.04(\mathrm{t}, J=7.2 \mathrm{~Hz}, 3 \mathrm{H}) .{ }^{13} \mathrm{C} \mathrm{NMR}\left(150 \mathrm{MHz}, \mathrm{CDCl}_{3}\right) \delta 194.90,193.15,164.61,134.67,133.15$, 132.34, 129.86, 128.90, 125.76, 115.17, 114.75, 69.90, 22.32, 10.25. HRMS (ESI): $\mathrm{m} / \mathrm{z}[\mathrm{M}+\mathrm{H}]^{+}$calcd for $\mathrm{C}_{17} \mathrm{H}_{16} \mathrm{O}_{3}: 269.1172$; found: 269.1180 .

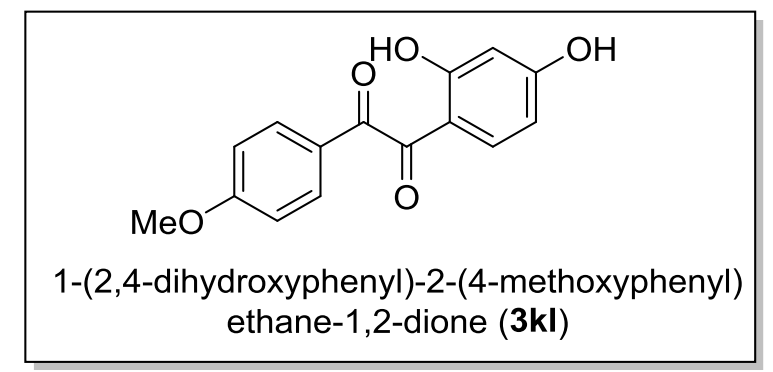

Yellow solid; $m p=151-152{ }^{\circ} \mathrm{C}$; IR $(\mathrm{KBr}) v_{\max }: 3435,2924,2850,1633,1601,1509,1453,1356,1310$, 1259, 1229, 1176, 1130, 1023, 978, 877, 841, 797, 639, 615, $514 \mathrm{~cm}^{-1} ;{ }^{1} \mathrm{H}$ NMR $(600 \mathrm{MHz}$, acetic 
acid- $\left.d_{4}\right) \delta 7.95(\mathrm{~d}, J=7.8 \mathrm{~Hz}, 2 \mathrm{H}), 7.36(\mathrm{~d}, J=8.4 \mathrm{~Hz}, 1 \mathrm{H}), 7.04(\mathrm{~d}, J=7.8 \mathrm{~Hz}, 2 \mathrm{H}), 6.47(\mathrm{~s}, 1 \mathrm{H}), 6.41$ $(\mathrm{d}, J=8.4 \mathrm{~Hz}, 1 \mathrm{H}), 3.89$ (s, 3H). ${ }^{13} \mathrm{C}$ NMR $\left(100 \mathrm{MHz}\right.$, acetic acid- $\left.d_{4}\right) \delta 198.93,192.84,167.70,167.64$, 166.99, 136.48, 134.24, 127.87, 116.26, 110.90, 105.13, 57.17. HRMS (ESI): $\mathrm{m} / \mathrm{z}[\mathrm{M}+\mathrm{Na}]^{+}$calcd for $\mathrm{C}_{15} \mathrm{H}_{12} \mathrm{NaO}_{5}$ : 295.0577; found: 295.0574 .

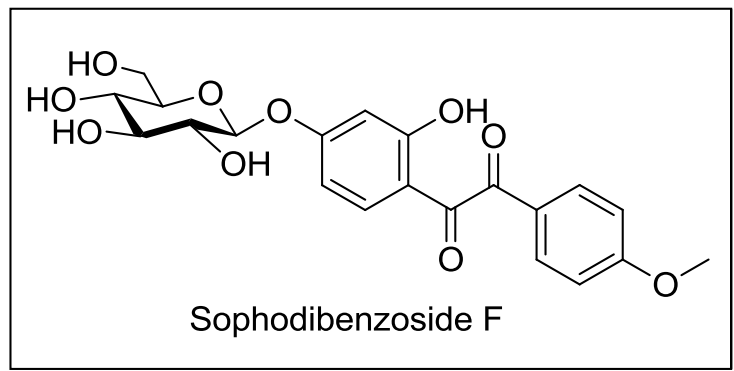

yellow powder; $\mathrm{mp}=201-203{ }^{\circ} \mathrm{C}$; [ a $]^{\mathrm{D}}{ }_{20}-84(\mathrm{c} 0.05, \mathrm{MeOH})$; IR $(\mathrm{KBr}) v_{\max }: 3395,2897,1658,1630$, 1601, 1572, 1505, 1425, 1401, 1313, 1262, 1231, 1180, 1125, 1089, 1035, 878, 842, 763, 664, 638, 612 $\mathrm{cm}^{-1} ;{ }^{1} \mathrm{H}$ NMR $\left(300 \mathrm{MHz}\right.$, acetic acid- $\left.d_{4}\right) \delta 7.96(\mathrm{~d}, J=8.7 \mathrm{~Hz}, 2 \mathrm{H}), 7.46(\mathrm{~d}, J=9.0 \mathrm{~Hz}, 1 \mathrm{H}), 7.05(\mathrm{~d}, J$ $=9.0 \mathrm{~Hz}, 2 \mathrm{H}), 6.71(\mathrm{~d}, J=2.1 \mathrm{~Hz}, 1 \mathrm{H}), 6.61(\mathrm{~d}, J=9.0 \mathrm{~Hz}, 1 \mathrm{H}), 5.22(\mathrm{~d}, J=7.2 \mathrm{~Hz}, 1 \mathrm{H}), 4.01(\mathrm{~m}$, , 1H), $3.90(\mathrm{~s}, 3 \mathrm{H}), 3.87-3.65(\mathrm{~m}, 5 \mathrm{H}) .{ }^{13} \mathrm{C}$ NMR $\left(100 \mathrm{MHz}\right.$, acetic acid- $\left.d_{4}\right) \delta 198.85,192.07,166.49$, $165.89,135.43,133.66,127.03,115.72,113.61,110.58,105.47,100.44,77.29,74.58,70.87,62.41$, 56.61. HRMS (ESI): $\mathrm{m} / \mathrm{z}[\mathrm{M}+\mathrm{H}]^{+}$calcd for $\mathrm{C}_{21} \mathrm{H}_{23} \mathrm{O}_{10}$ : 435.1286; found: 435.1288 .

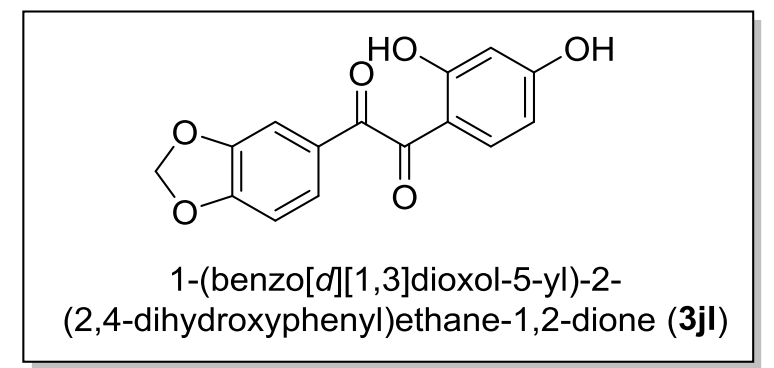

Yellow solid; $\mathrm{mp}=167-169{ }^{\circ} \mathrm{C}$; IR $(\mathrm{KBr}) v_{\max }: 3201,2919,1633,1594,1503,1453,1353,1260,1187$, $1110,1037,977,930,850,802,765,718,622,580,556,478 \mathrm{~cm}^{-1} ;{ }^{1} \mathrm{H}$ NMR $\left(600 \mathrm{MHz}\right.$, acetic acid- $\left.d_{4}\right)$ $\delta 7.51(\mathrm{~d}, J=7.8 \mathrm{~Hz}, 1 \mathrm{H}), 7.46(\mathrm{~s}, 1 \mathrm{H}), 7.34(\mathrm{~d}, J=9.0 \mathrm{~Hz}, 1 \mathrm{H}), 6.91(\mathrm{~d}, J=8.4 \mathrm{~Hz}, 1 \mathrm{H}), 6.45(\mathrm{~s}, 1 \mathrm{H})$, $6.41(\mathrm{~d}, J=9.0 \mathrm{~Hz}, 1 \mathrm{H}), 6.09(\mathrm{~s}, 2 \mathrm{H}) .{ }^{13} \mathrm{C}$ NMR $\left(150 \mathrm{MHz}\right.$, acetic acid- $\left.d_{4}\right) \delta 198.533,192.34,167.54$, 167.52, 155.32, 150.40, 136.23, 129.72, 129.39, 112.25, 110.57, 109.80, 109.41, 104.77, 104.17. HRMS (ESI): $\mathrm{m} / \mathrm{z}[\mathrm{M}+\mathrm{H}]^{+}$calcd for $\mathrm{C}_{15} \mathrm{H}_{11} \mathrm{O}_{6}: 287.0550$; found: 287.0500 .

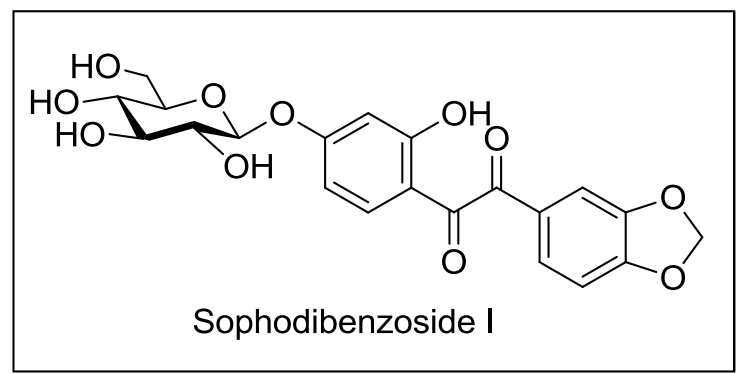


yellow powder; $\mathrm{mp}=145-148{ }^{\circ} \mathrm{C}$; [ a $]^{\mathrm{D}} 20-60$ (c 0.025, MeOH); IR (KBr) $v_{\max }: 3396,2962,2924$, 1663, 1627, 1505, 1441, 1391, 1258, 1182, 1097, 1073, 1043, 1020, 930, 842, 804, $634 \mathrm{~cm}^{-1}$; ${ }^{1} \mathrm{H}$ NMR (300 MHz, acetic acid- $\left.d_{4}\right) \delta 7.51(\mathrm{dd}, J=8.1,1.5 \mathrm{~Hz}, 1 \mathrm{H}), 7.46(\mathrm{~m}, 2 \mathrm{H}), 6.93(\mathrm{~d}, J=8.1 \mathrm{~Hz}, 1 \mathrm{H}), 6.70$ $(\mathrm{d}, J=2.4 \mathrm{~Hz}, 1 \mathrm{H}), 6.61(\mathrm{dd}, J=9.0,2.4 \mathrm{~Hz}, 1 \mathrm{H}), 6.11(\mathrm{~s}, 2 \mathrm{H}), 5.22(\mathrm{~d}, J=7.5 \mathrm{~Hz}, 1 \mathrm{H}), 4.02(\mathrm{~m}, 1 \mathrm{H})$, $3.96-3.57(\mathrm{~m}, 6 \mathrm{H}) .{ }^{13} \mathrm{C}$ NMR $\left(100 \mathrm{MHz}\right.$, acetic acid- $\left.d_{4}\right) \delta 198.51,191.62,166.48,165.93,155.05$, 150.08, 135.41, 129.45, 128.89, 113.60, 110.62, 109.60, 109.18, 105.49, 103.97, 100.48, 77.30, 74.58, 70.90, 62.43. HRMS (ESI): $\mathrm{m} / \mathrm{z}[\mathrm{M}+\mathrm{H}]^{+}$calcd for $\mathrm{C}_{21} \mathrm{H}_{21} \mathrm{O}_{11}$ : 449.1078; found: 449.1075 . 


\section{Crystallographic data and molecular structures}

\subsection{1-(4-hydroxy-3-methylphenyl)-2-phenylethane-1,2-dione (3aa)}

$\mathrm{X}$-ray structure determination was obtained via slow evaporation of compound $\mathbf{3 a a}$ in $\mathrm{CHCl}_{3} / \mathrm{MeOH}$ (1:1) at room temperature.

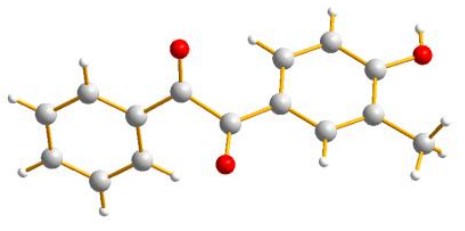<smiles>Cc1cc(C(=O)C(=O)c2ccccc2)ccc1O</smiles>

Figure S5.1 X-ray crystal structure of 3aa;

Table S5.1 Crystal data and structure refinement for compound 3aa (CCDC: 1487105)

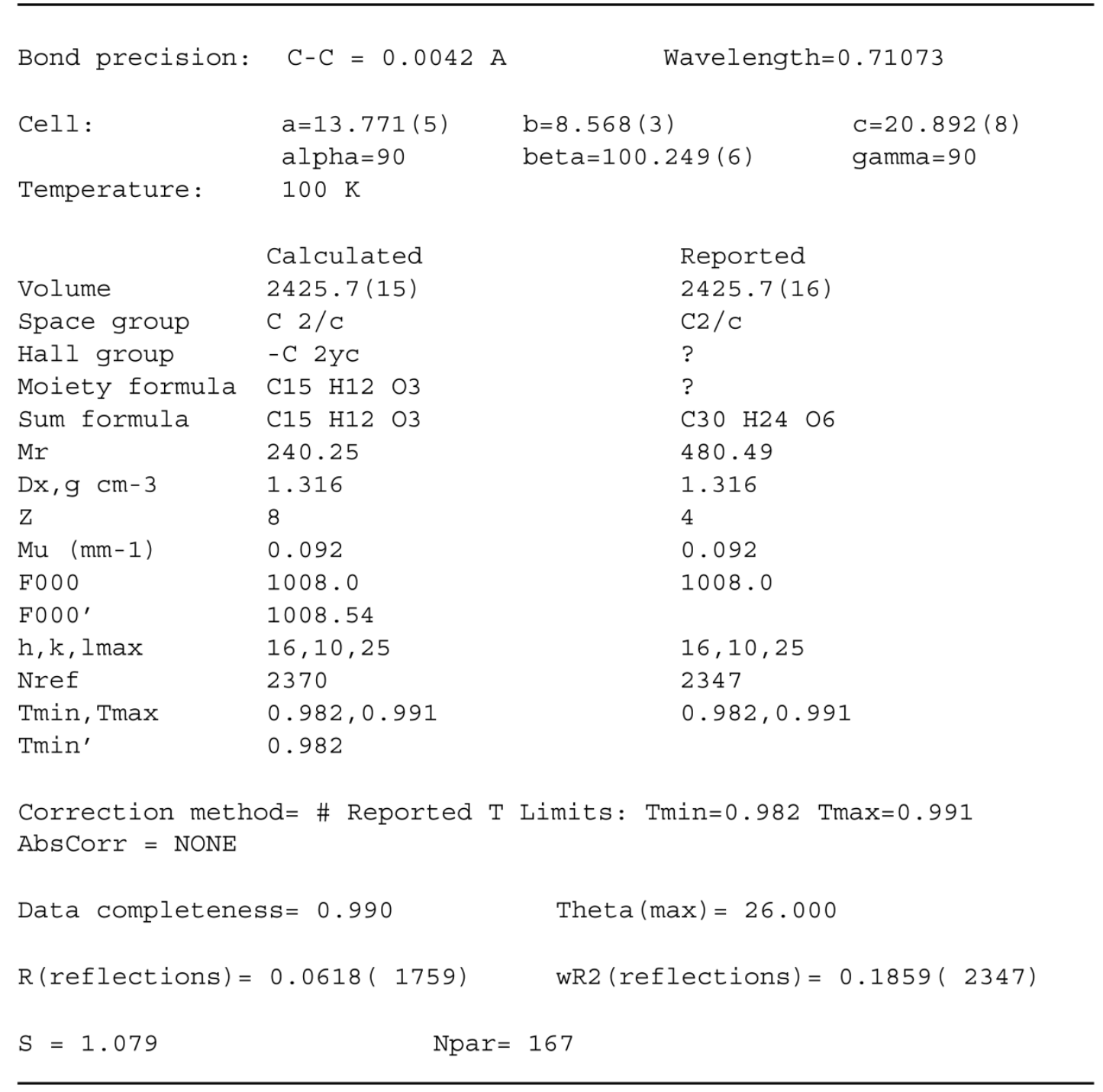




\subsection{1-(4-hydroxy-3,5-dimethylphenyl)-2-(naphthalen-2-yl)ethane-1,2-dione (3jb)}

$\mathrm{X}$-ray structure determination was obtained via slow evaporation of compound $\mathbf{3 j b}$ in $\mathrm{CHCl}_{3} / \mathrm{MeOH}$ $(4: 1)$ at room temperature.

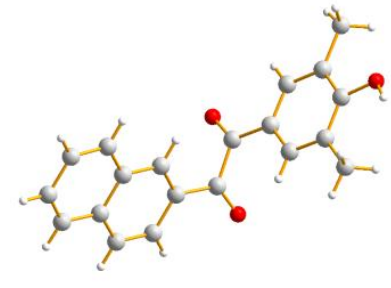<smiles>Cc1cc(C(=O)C(=O)c2ccc3ccccc3c2)cc(C)c1O</smiles>

Figure S5.2 X-ray crystal structure of $\mathbf{3 j b}$;

Table S5.2 Crystal data and structure refinement for compound 3jb (CCDC: 1487106)

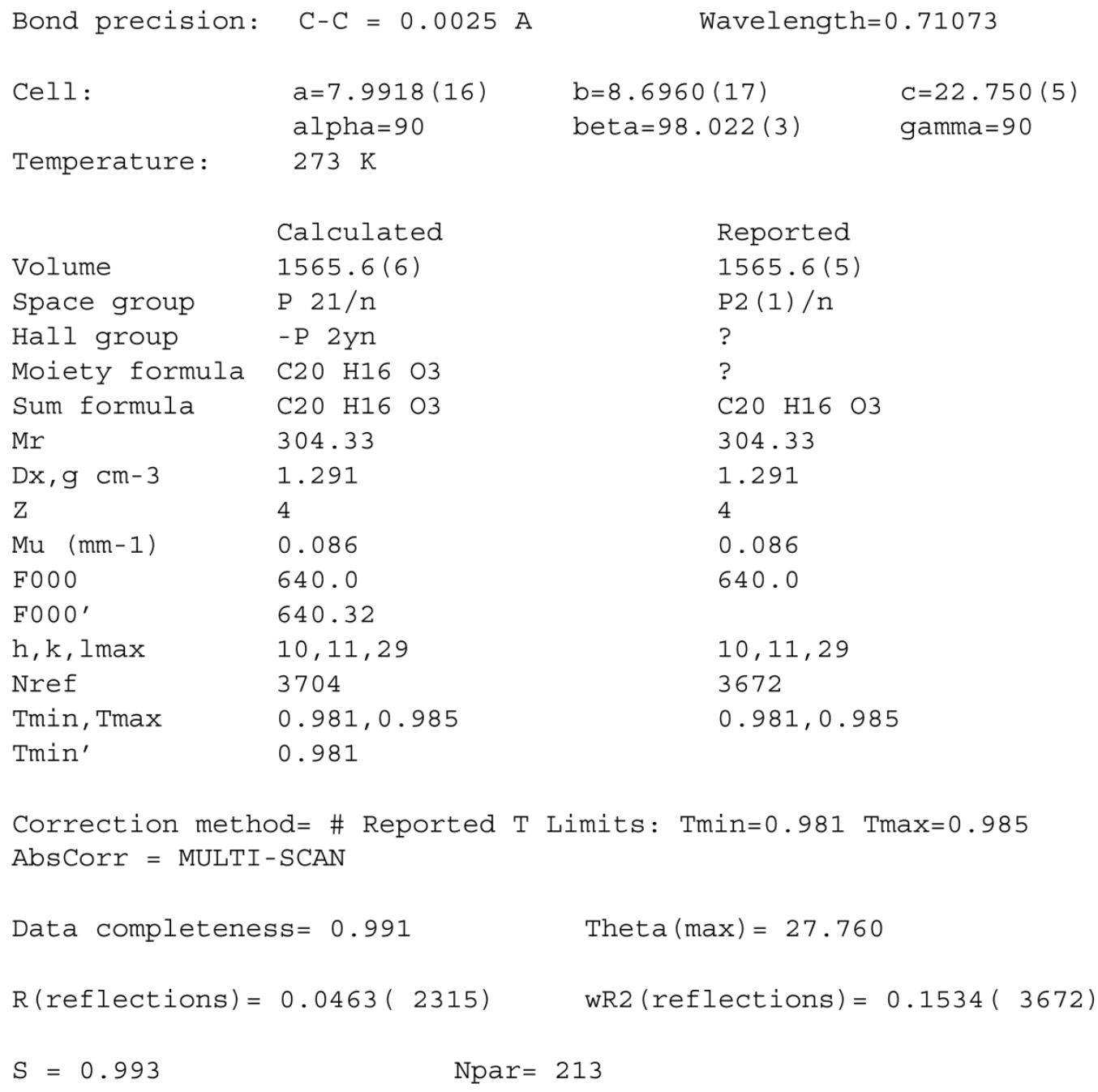




\subsection{1-(benzo[d][1,3]dioxol-5-yl)-2-(4-hydroxy-3,5-dimethylphenyl)ethane-1,2-dione} (3kb)

$\mathrm{X}$-ray structure determination was obtained via slow evaporation of compound $\mathbf{3 j b}$ in $\mathrm{CDCl}_{3}$ at room temperature.

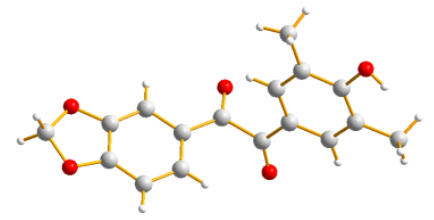<smiles>Cc1cc(C(=O)C(=O)c2ccc3c(c2)OCO3)cc(C)c1O</smiles>

Figure S5.3 X-ray crystal structure of $\mathbf{3 k b}$;

Table S5.3 Crystal data and structure refinement for compound 3kb (CCDC: 1487107)

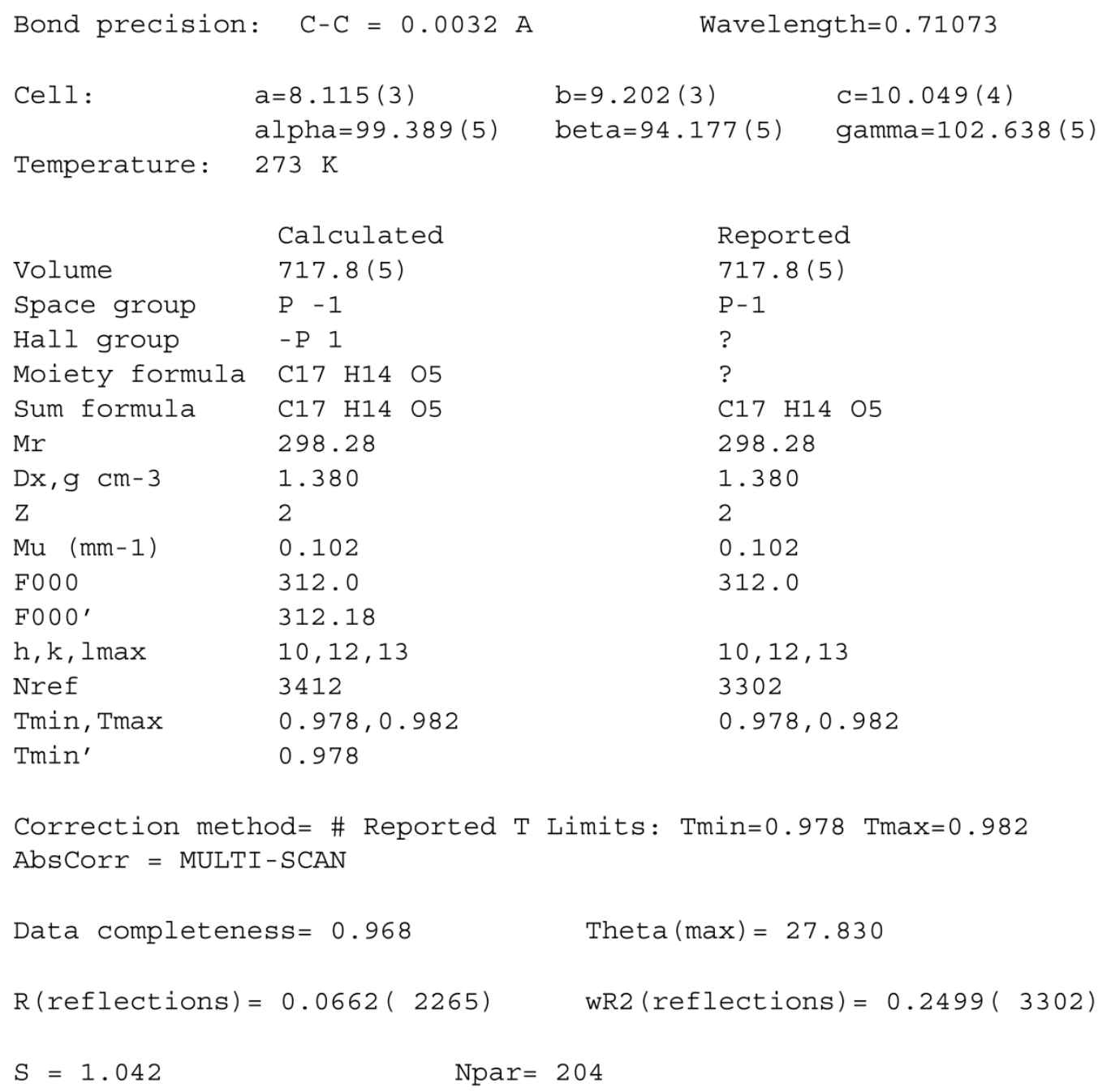

$\begin{array}{lll} & \text { Calculated } & \text { Reported } \\ \text { Volume } & 717.8(5) & 717.8(5) \\ \text { Space group } & \text { P }-1 & \text { P-1 } \\ \text { Hall group } & -\mathrm{P} 1 & ? \\ \text { Moiety formula } & \mathrm{C} 17 \mathrm{H} 14 \text { O5 } & ? \\ \text { Sum formula } & \mathrm{C} 17 \mathrm{H} 14 \text { O5 } & \text { C17 H14 O5 } \\ \text { Mr } & 298.28 & 298.28 \\ \text { Dx, g cm-3 } & 1.380 & 1.380 \\ \text { Z } & 2 & 2 \\ \text { Mu (mm-1) } & 0.102 & 0.102 \\ \text { F000 } & 312.0 & 312.0 \\ \text { F000' } & 312.18 & \\ \text { h, k, lmax } & 10,12,13 & 10,12,13 \\ \text { Nref } & 3412 & 3302 \\ \text { Tmin, Tmax } & 0.978,0.982 & 0.978,0.982 \\ \text { Tmin' } & 0.978 & \end{array}$

Correction method $=\#$ Reported T Limits: Tmin $=0.978$ Tmax $=0.982$ AbsCorr $=$ MULTI-SCAN

Data completeness $=0.968$

Theta $(\max )=27.830$

$\mathrm{R}($ reflections $)=0.0662(2265)$

$\mathrm{wR} 2($ reflections $)=0.2499(3302)$

$S=1.042$

Npar $=204$ 


\section{Copies of $\mathrm{H}$ NMR, "C NMR Spectra}

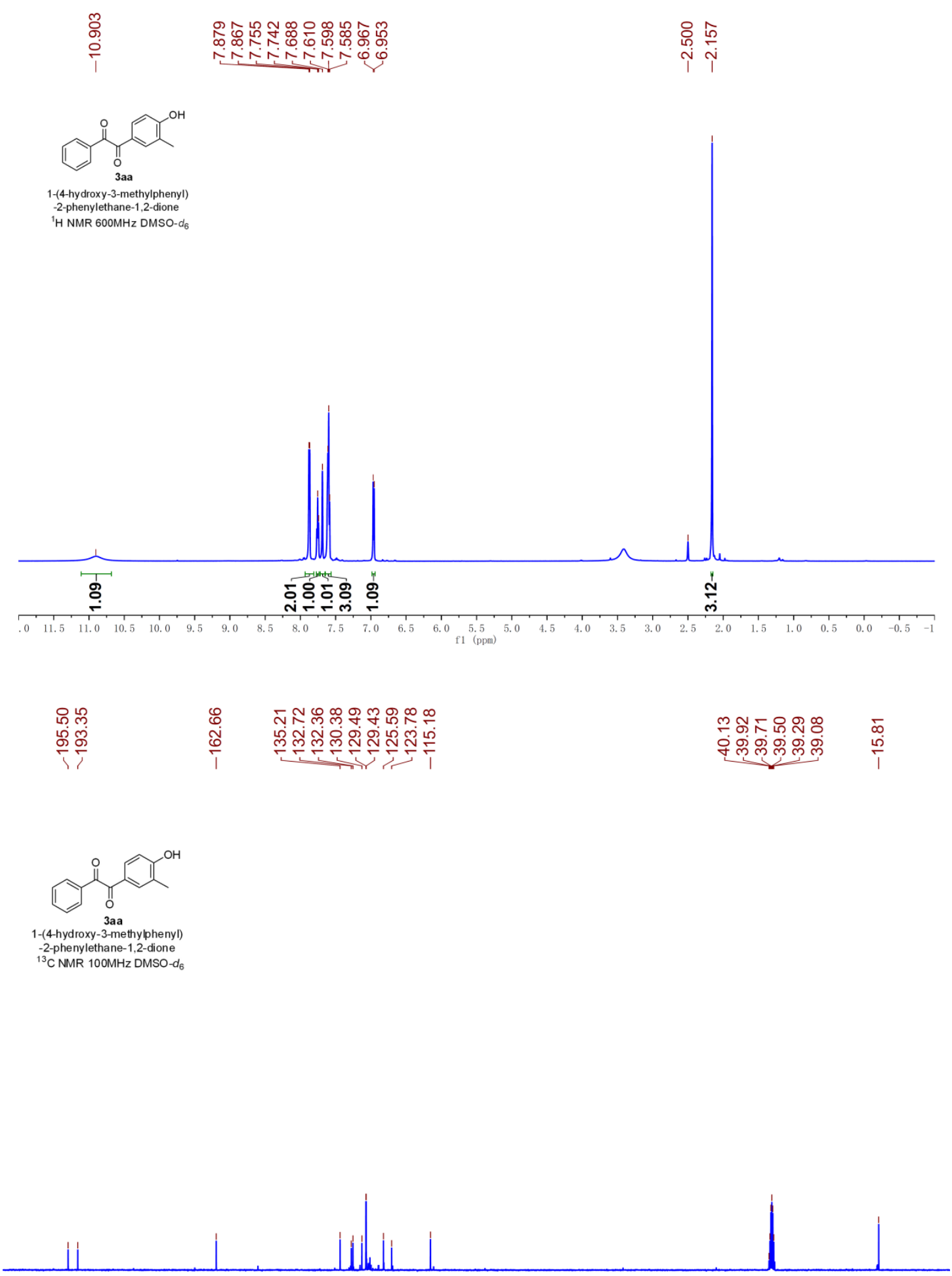

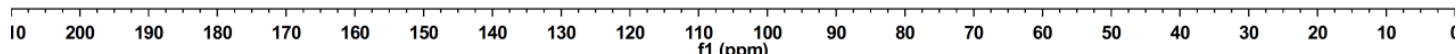




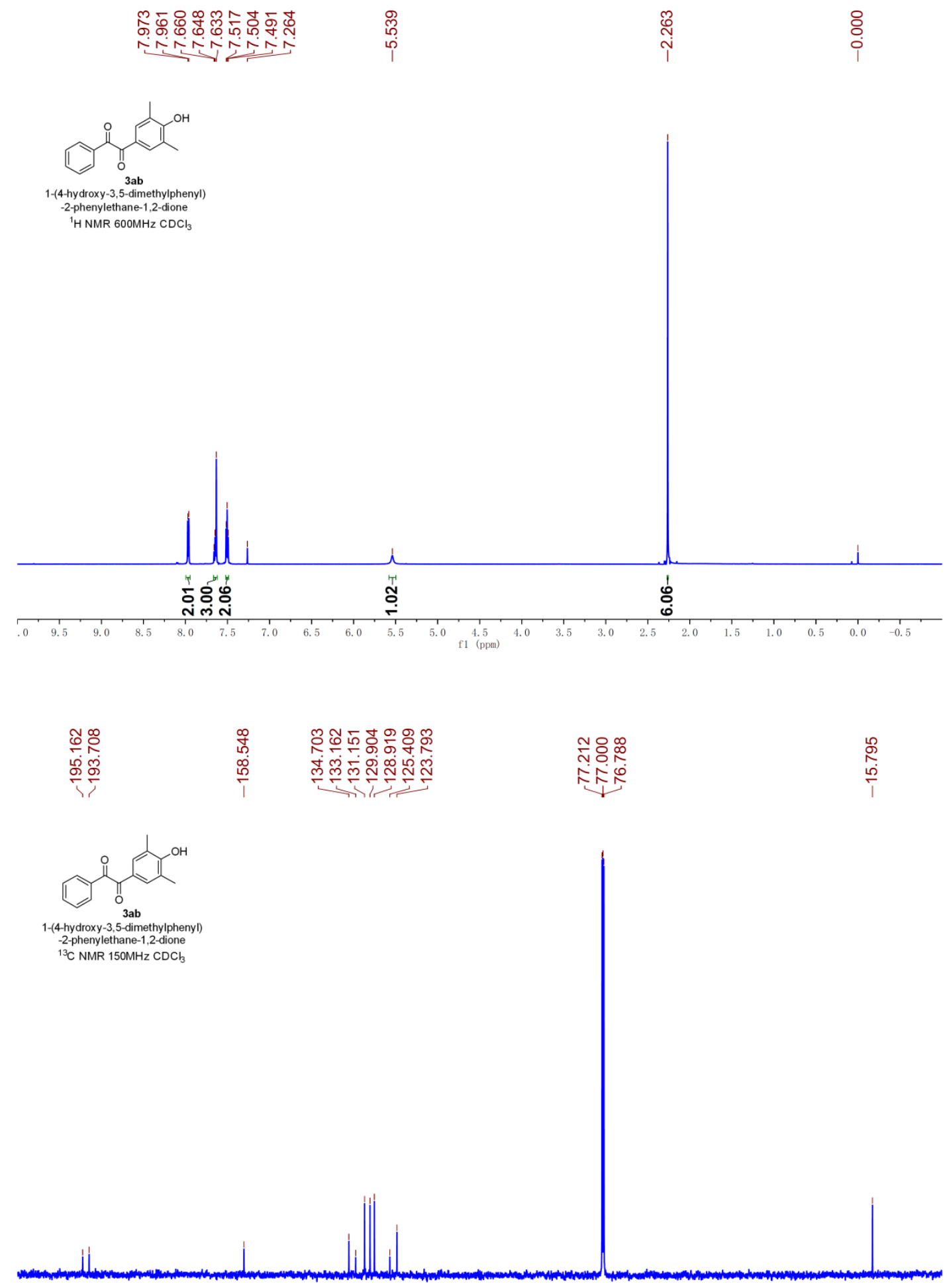

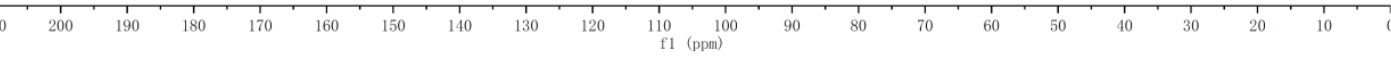



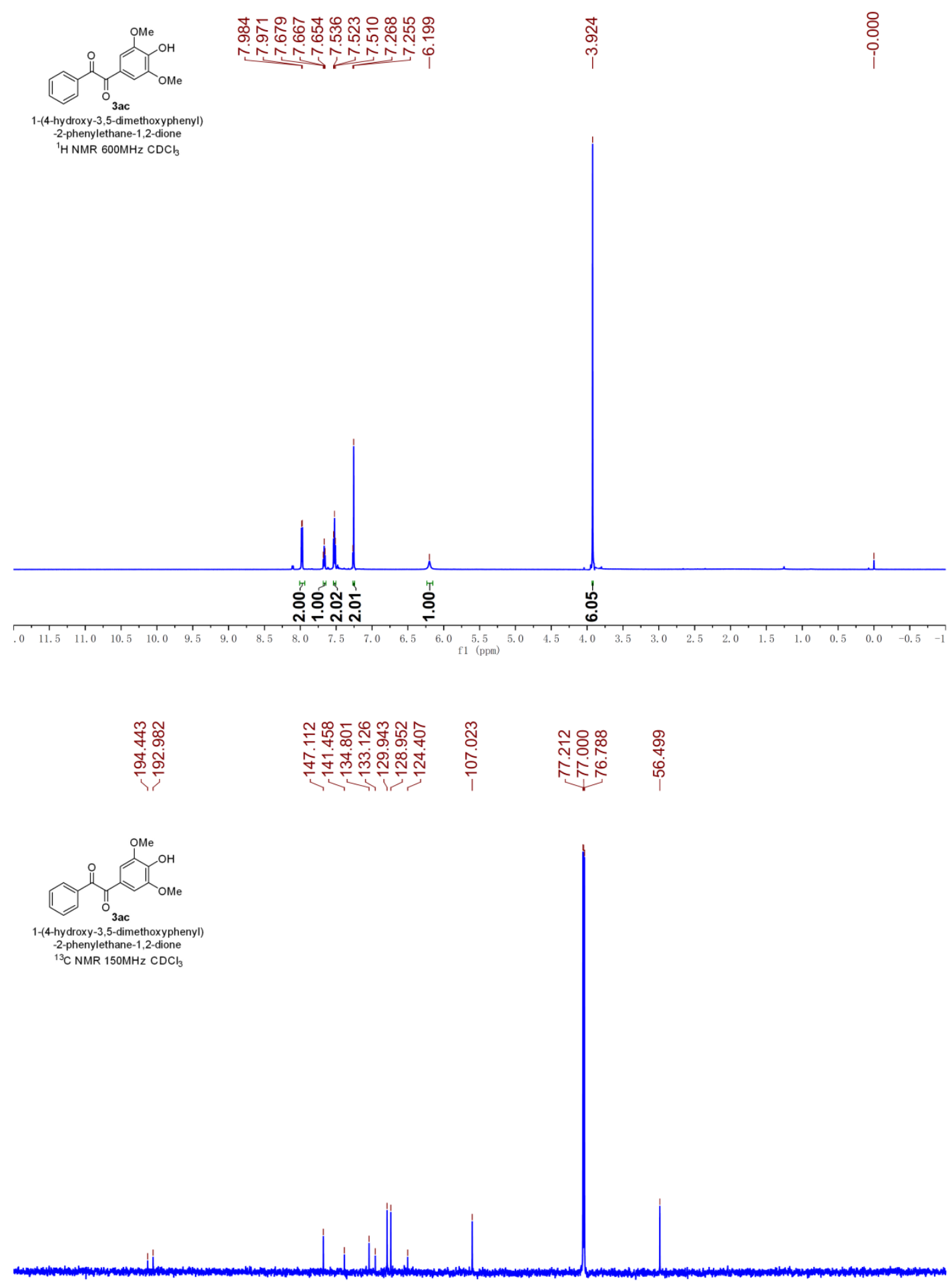

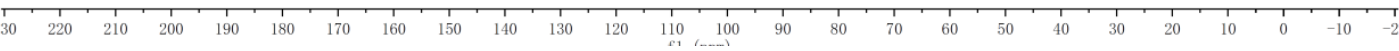




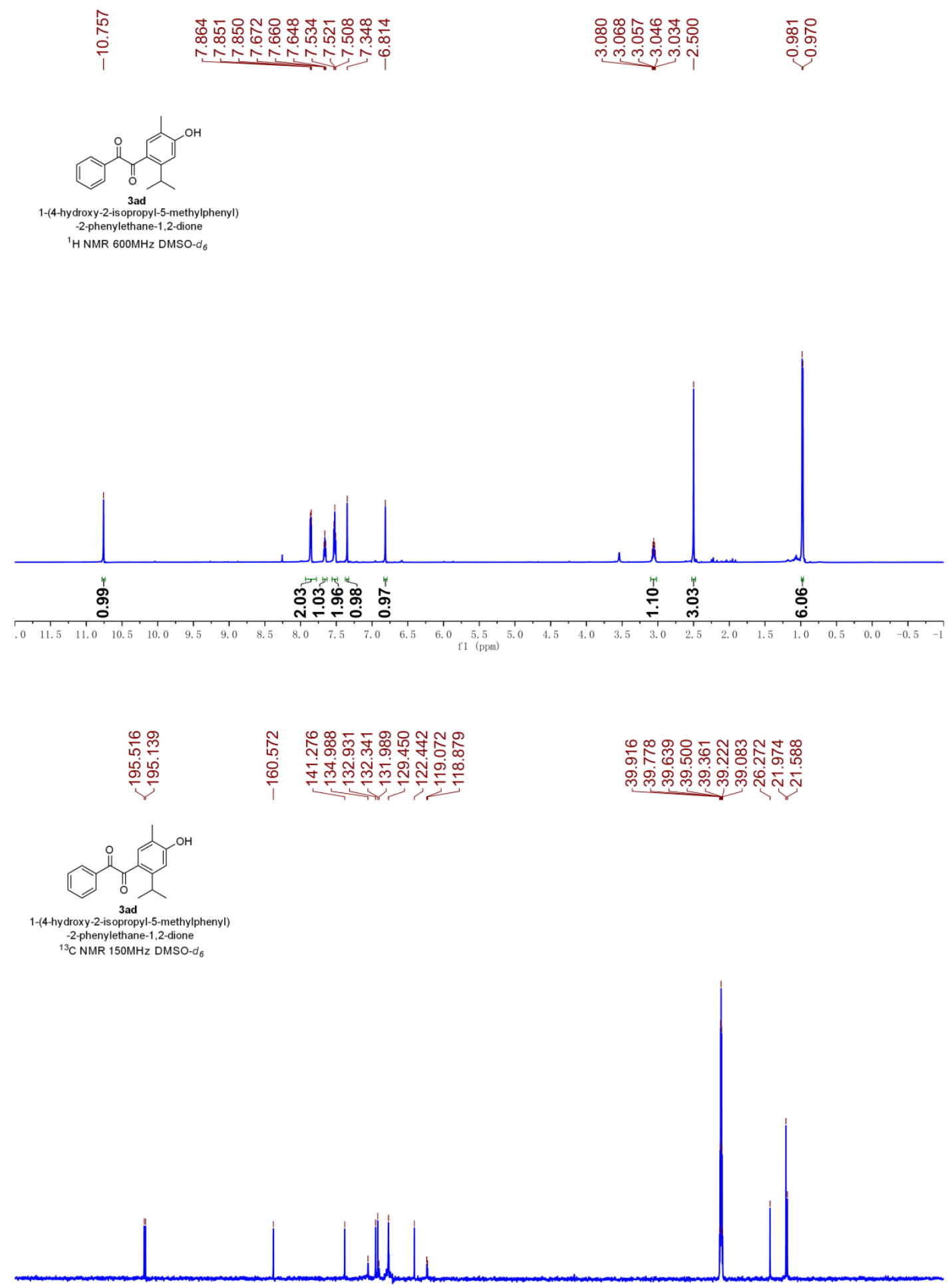

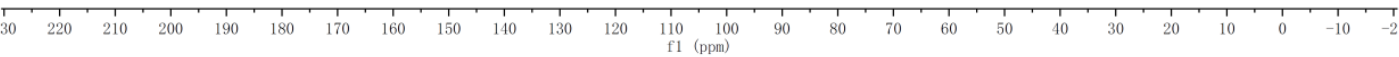



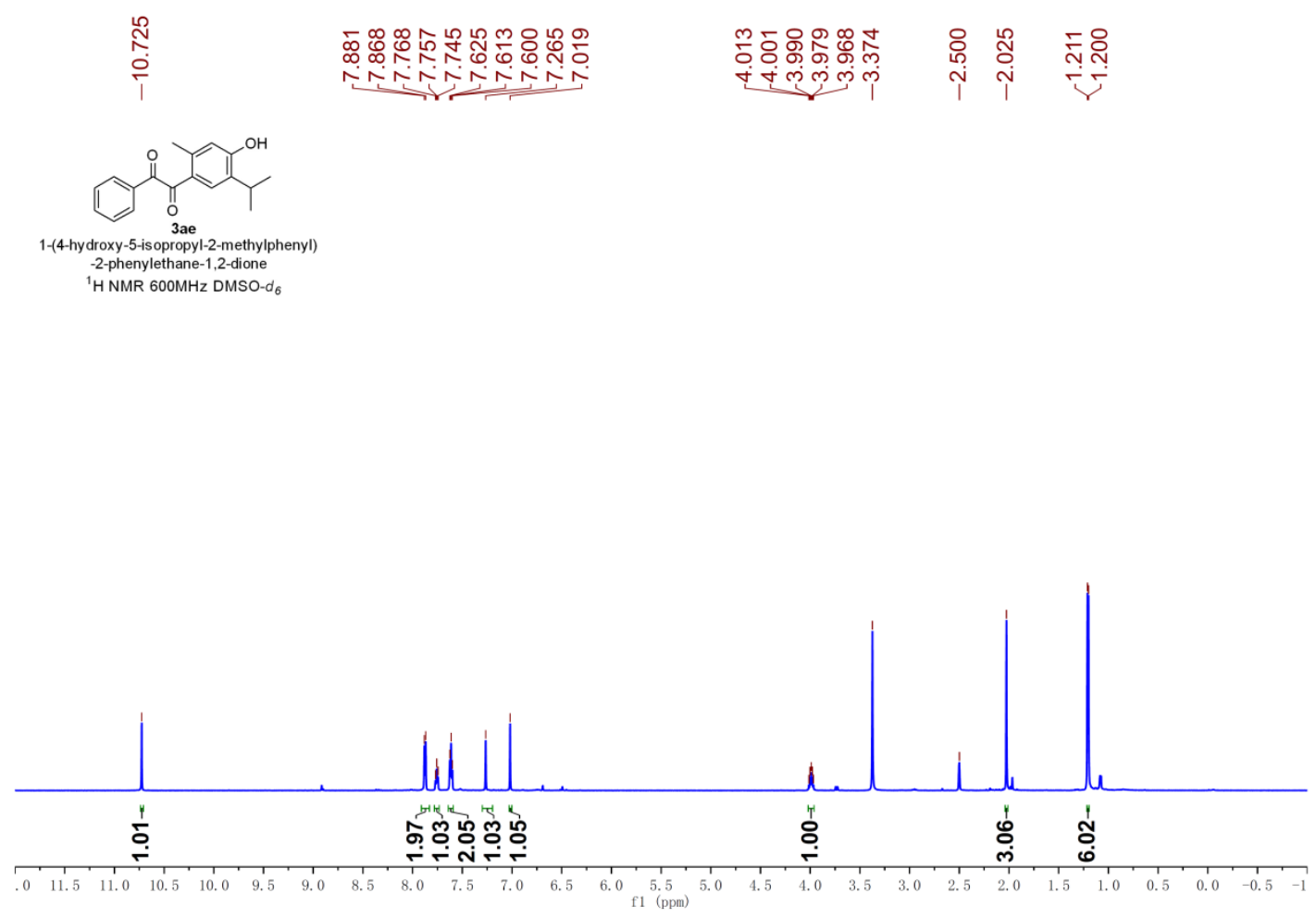

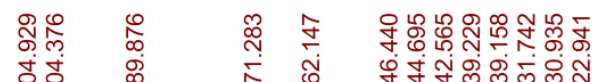

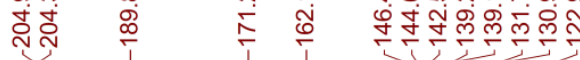

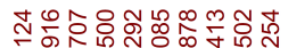

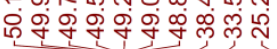

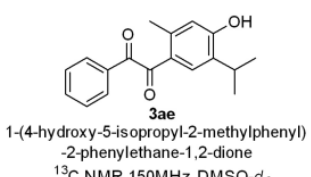

-2-phenylethane-1,2-dione
${ }^{13} \mathrm{C}$ NMR $150 \mathrm{MHz}$ DMSO- $d_{0}$

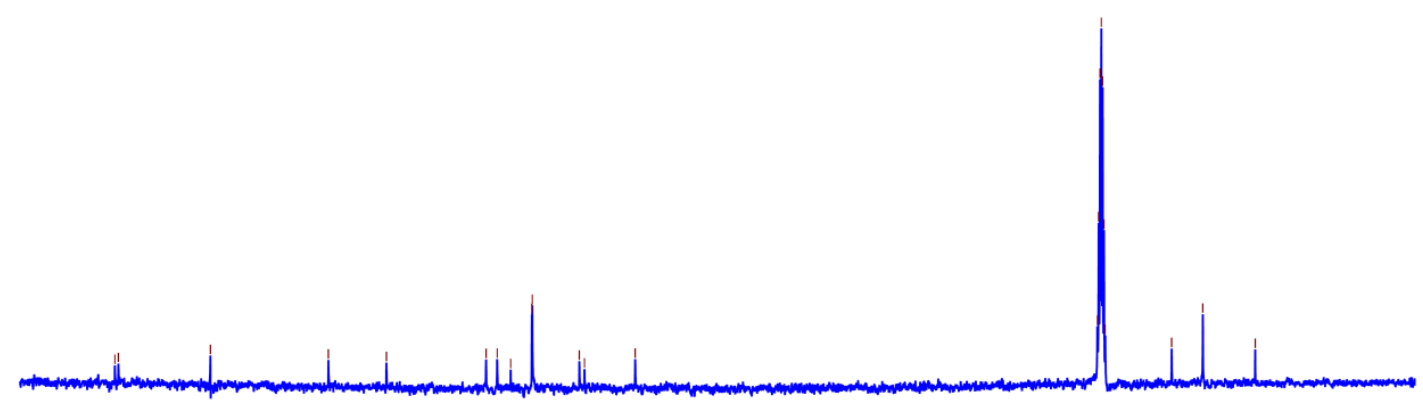

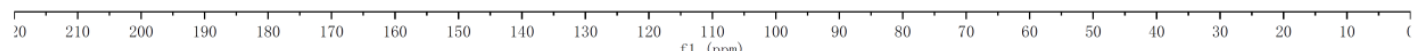




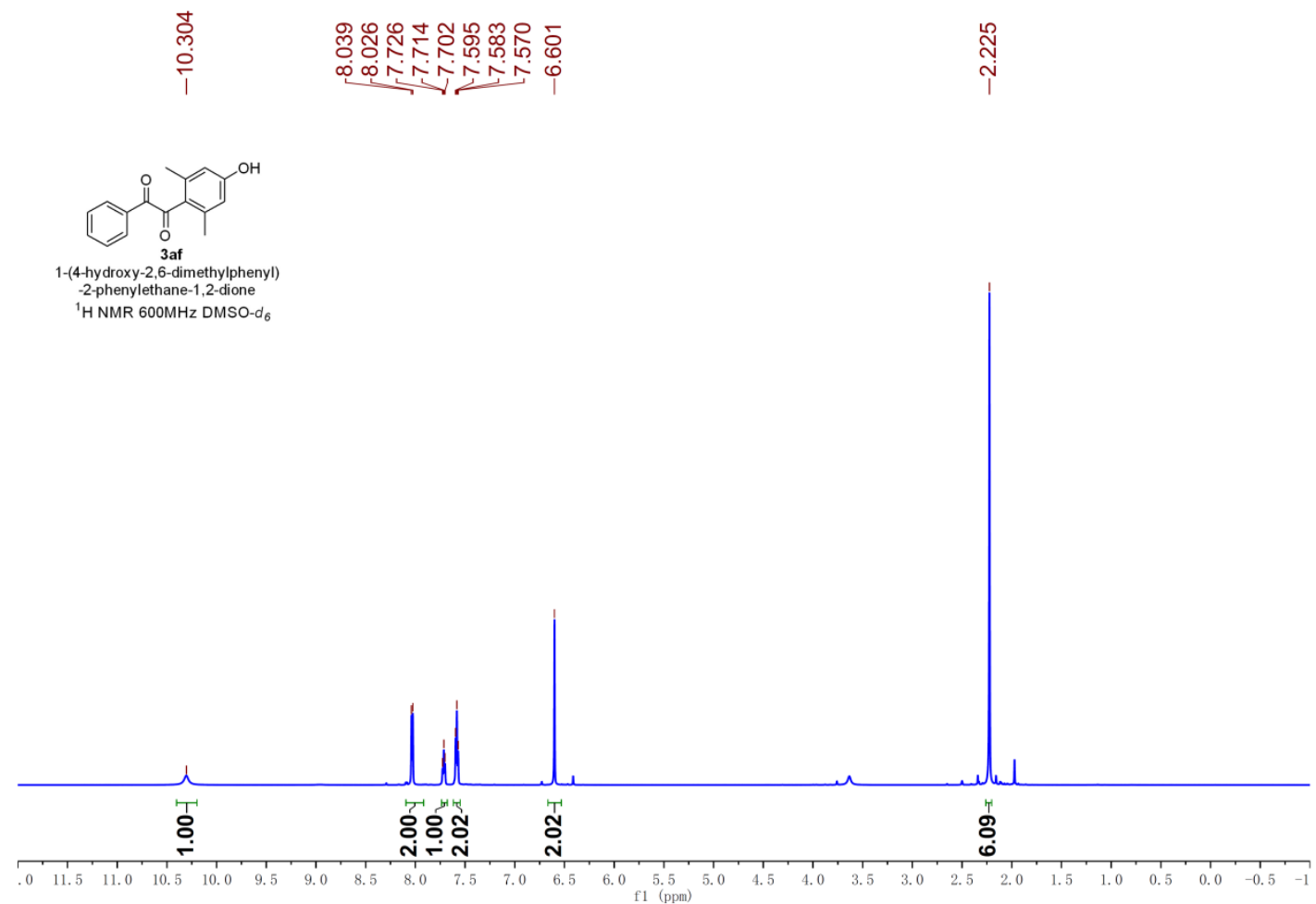

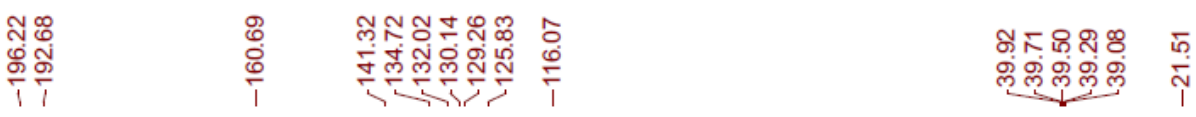
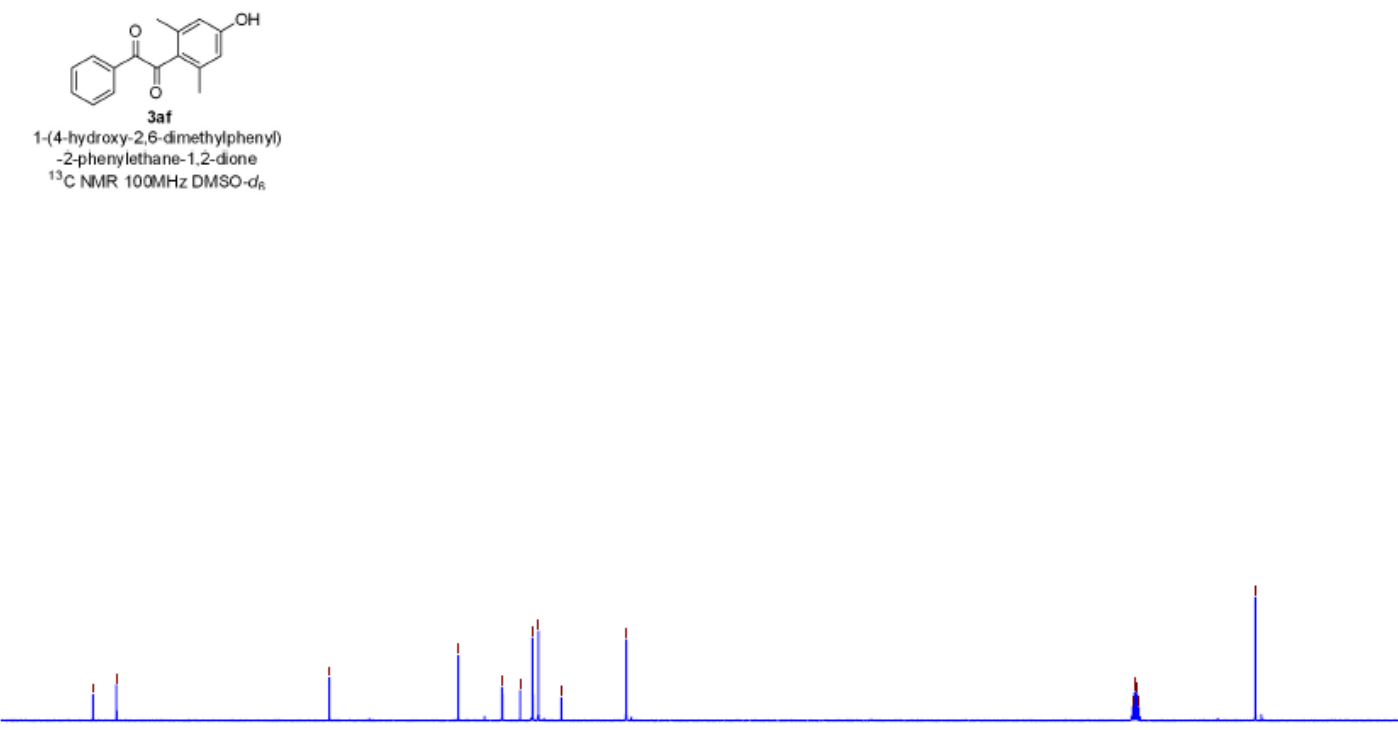

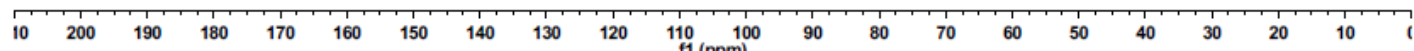




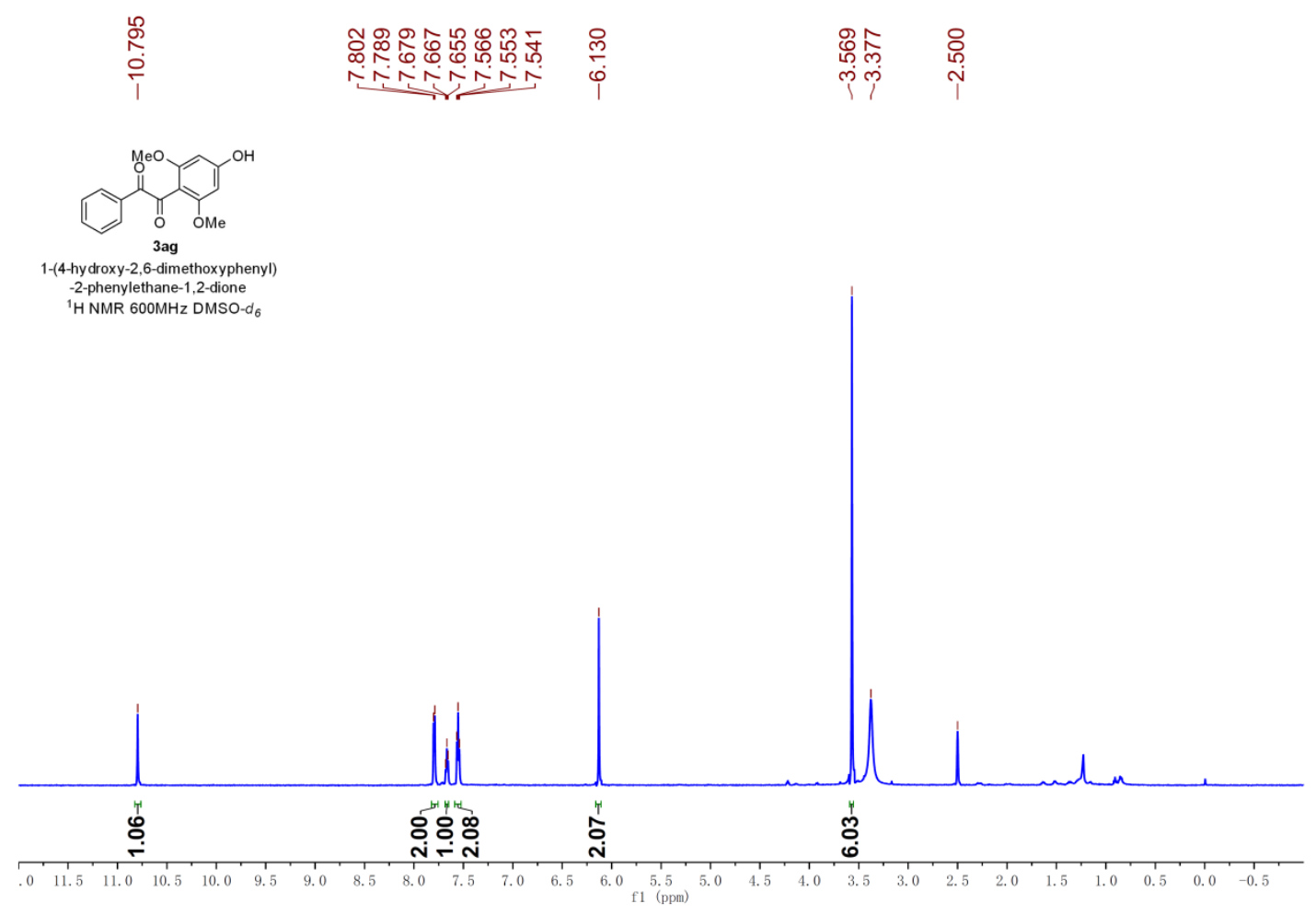

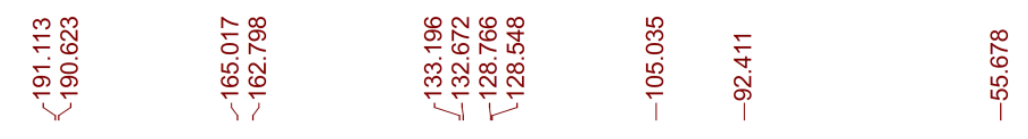

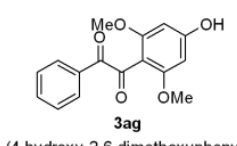

1-(4-hydroxy-2,6-dimethoxyphenyl)

${ }^{13} \mathrm{C}$ NMR 100MHz DMSO-d

$\infty$
0
$\substack{0 \\ 1}$

(n) 


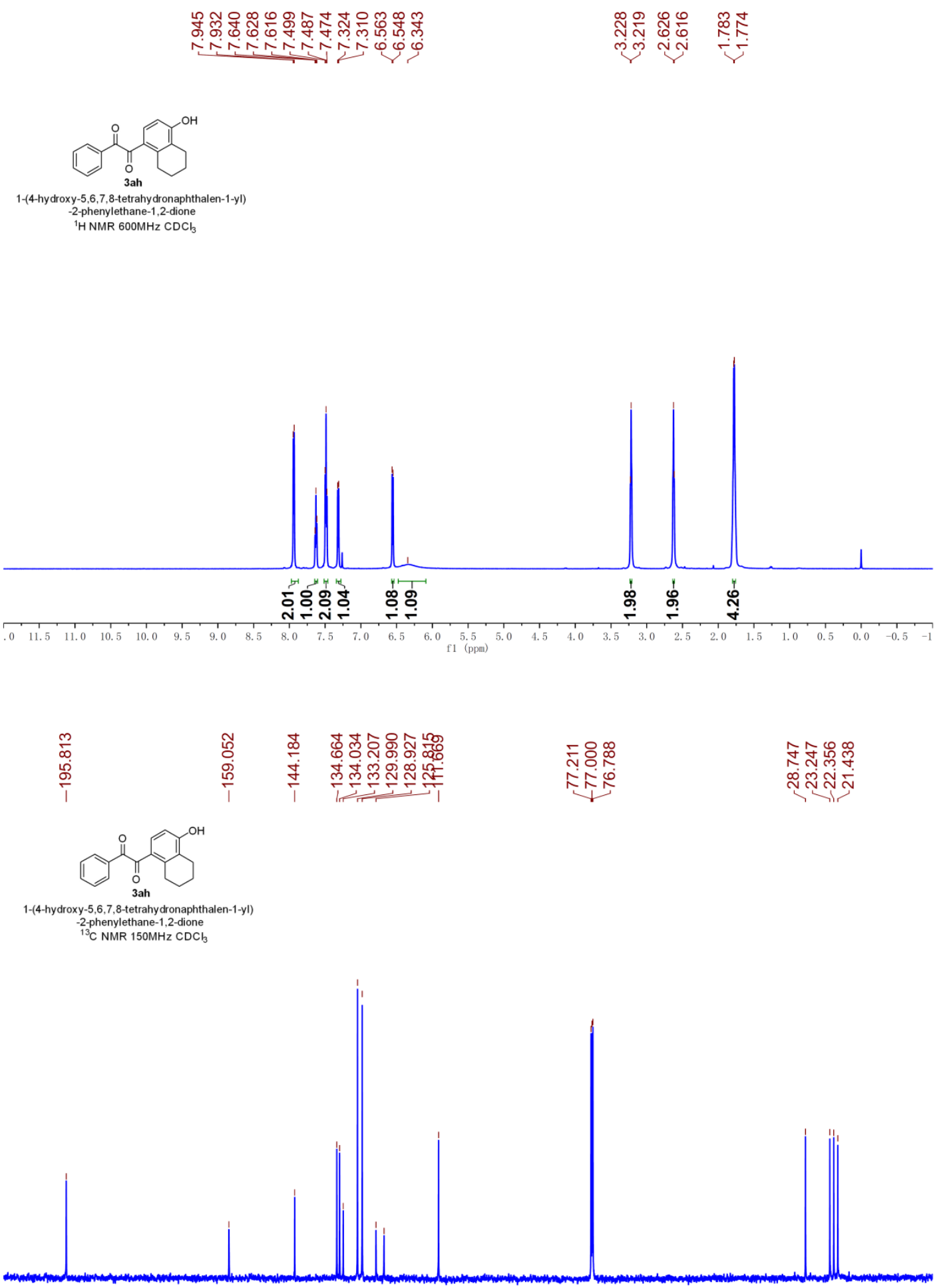

1-(4-hydroxy-5,6,7,8-tetrahydronapht

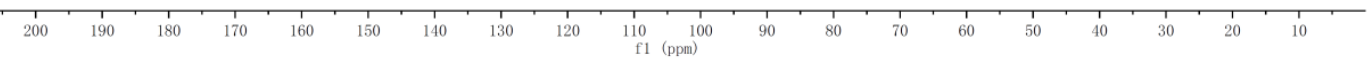



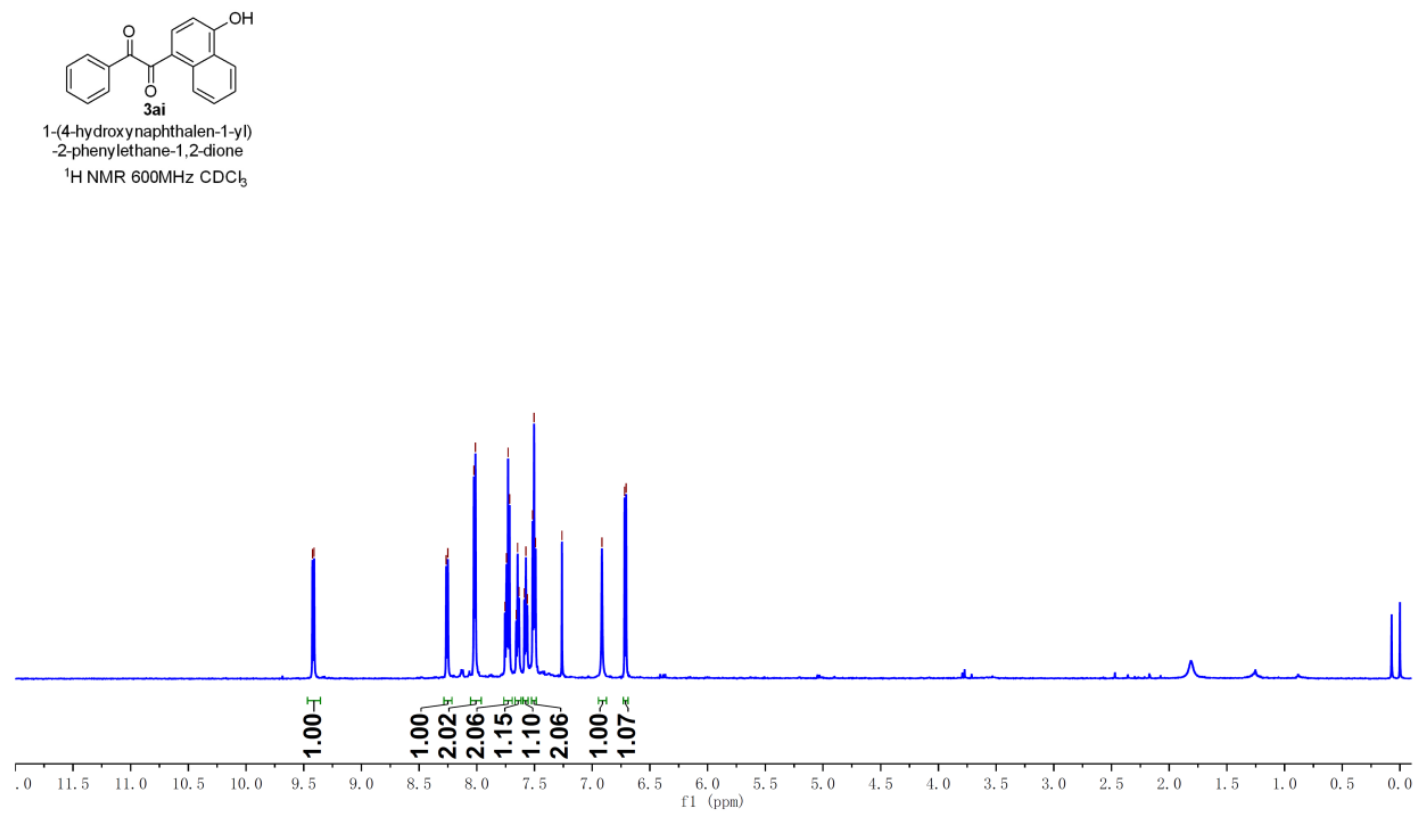

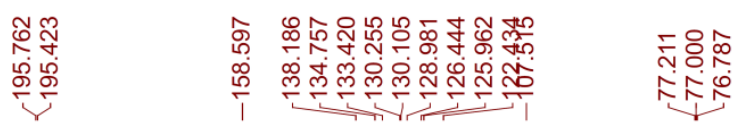

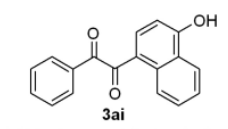

1-(4-hydroxynaphthalen-1-y

-2-phenylethane-1,2-dione

${ }^{3} \mathrm{C}$ NMR $150 \mathrm{MHz} \mathrm{CDC}$

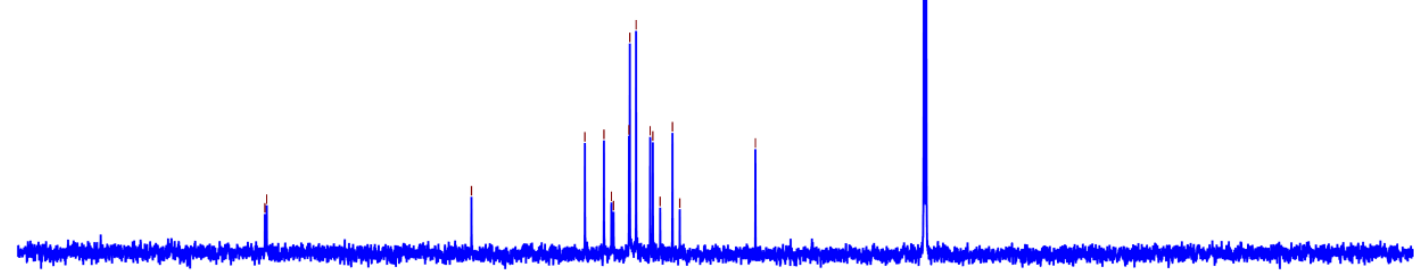

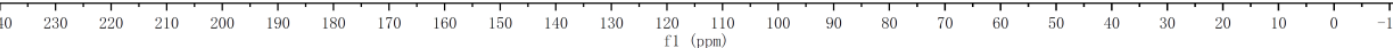




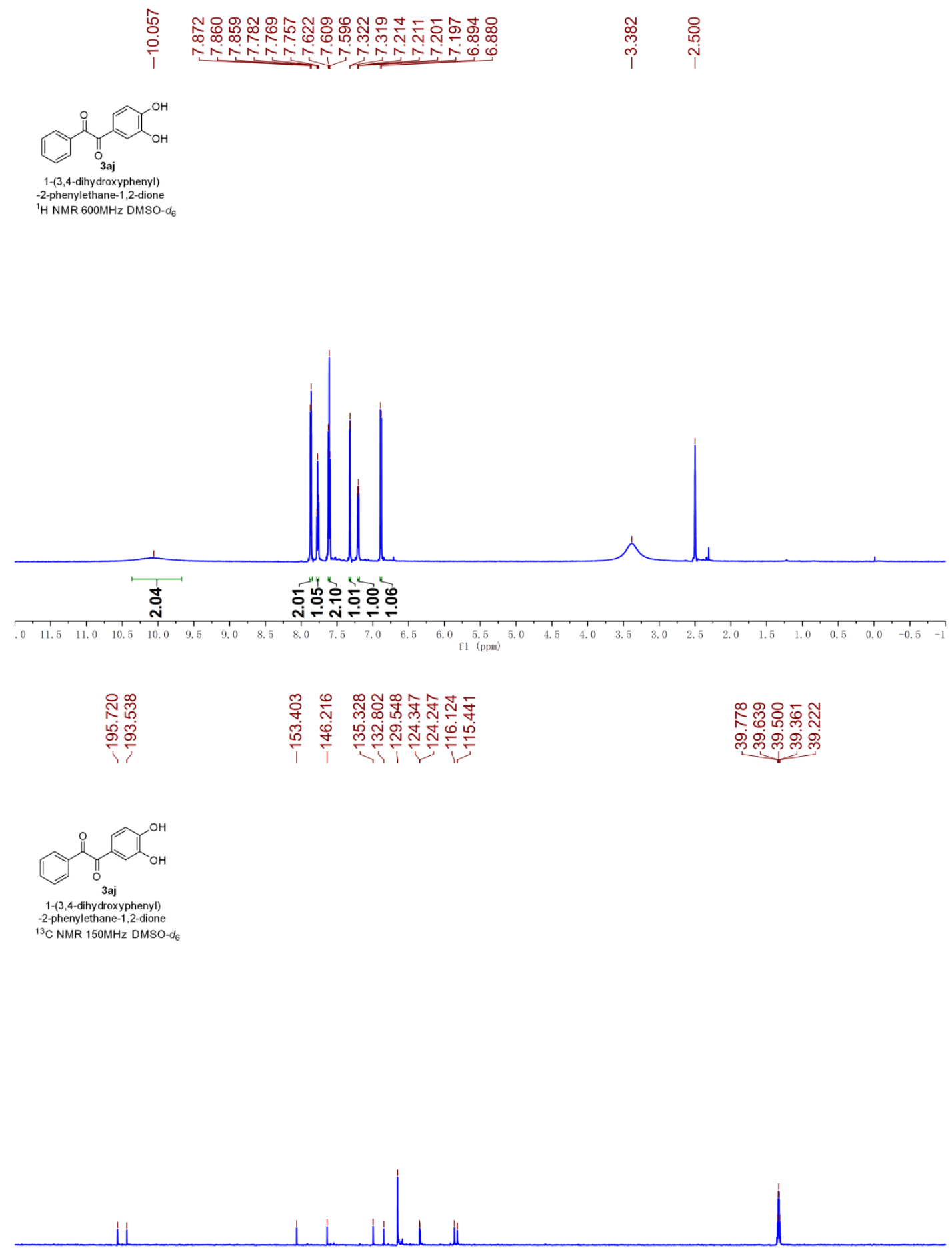

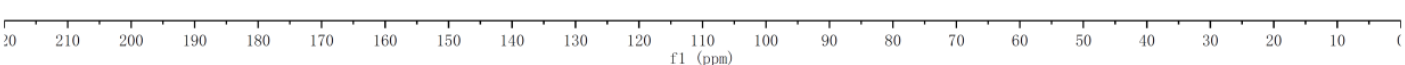




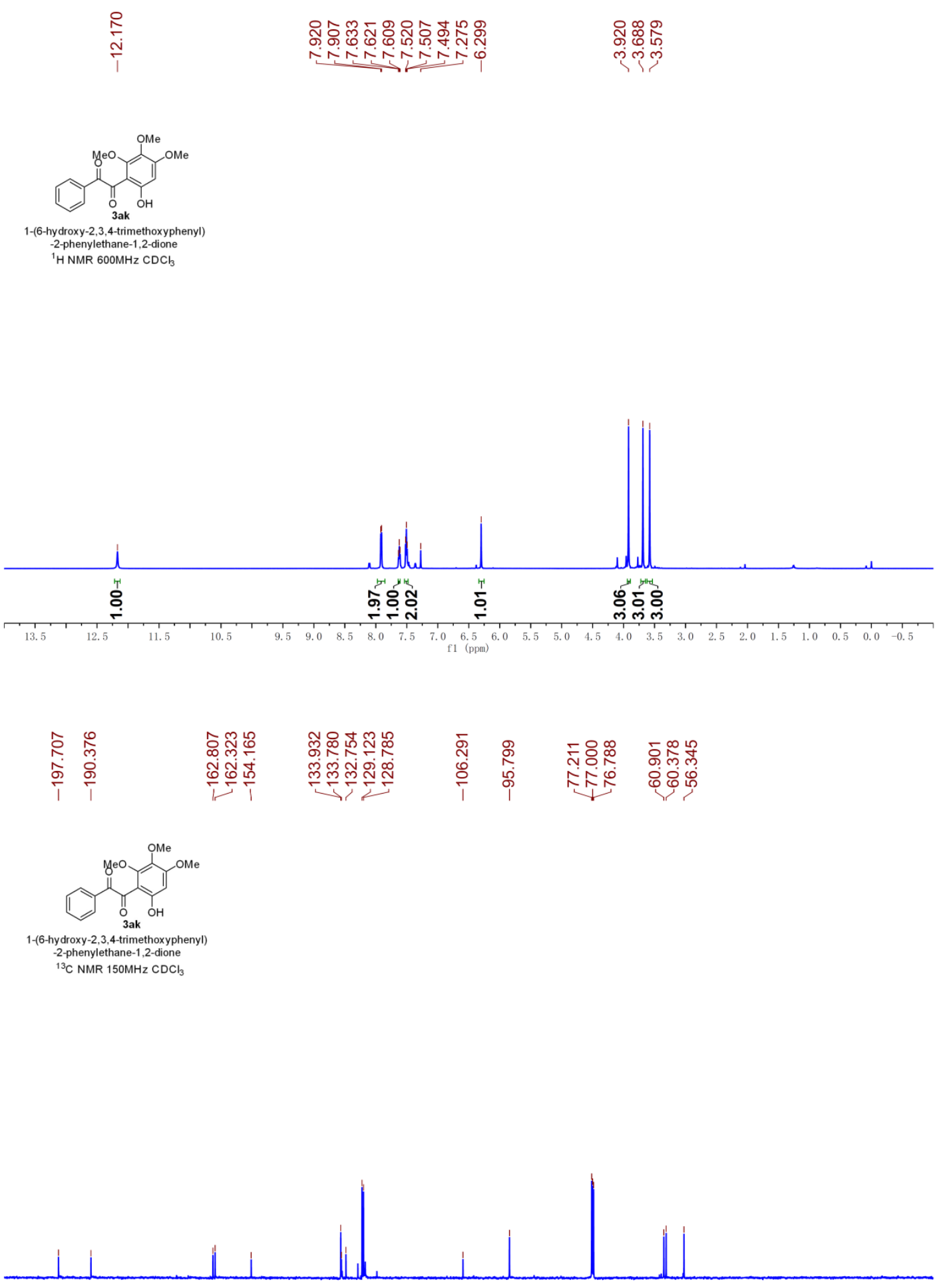

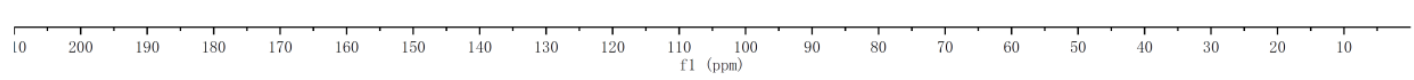



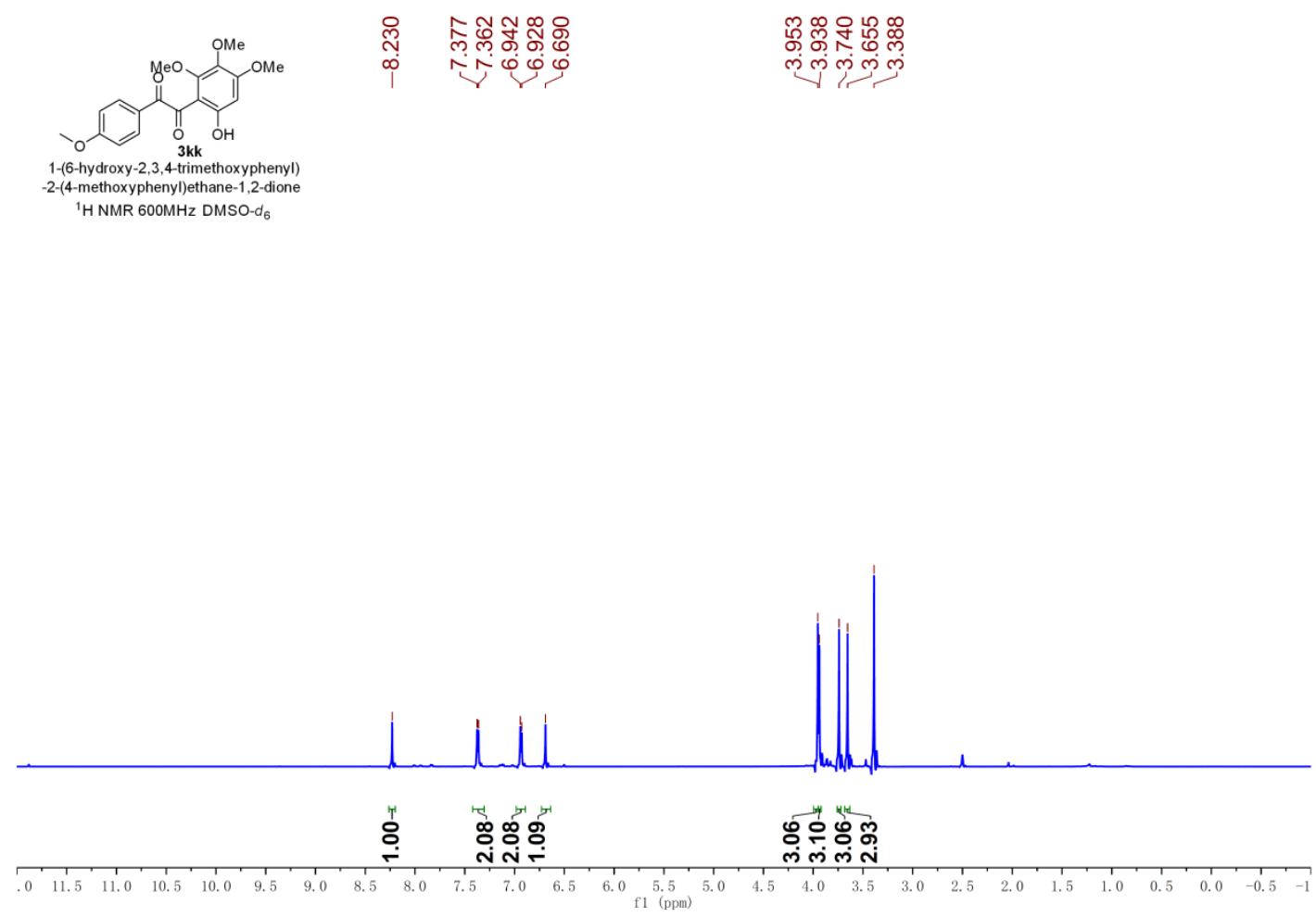

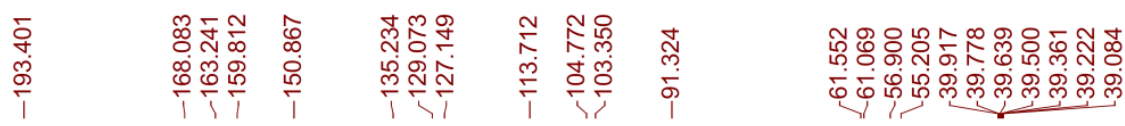

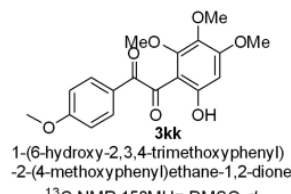

13. CNMR 150MHz DMSO-dio

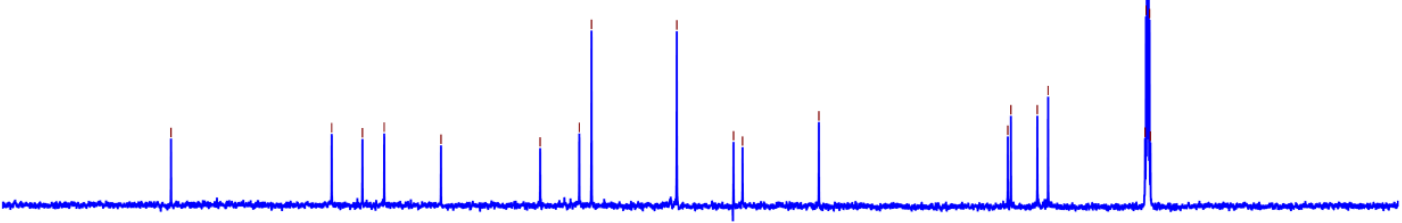

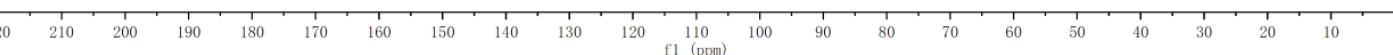



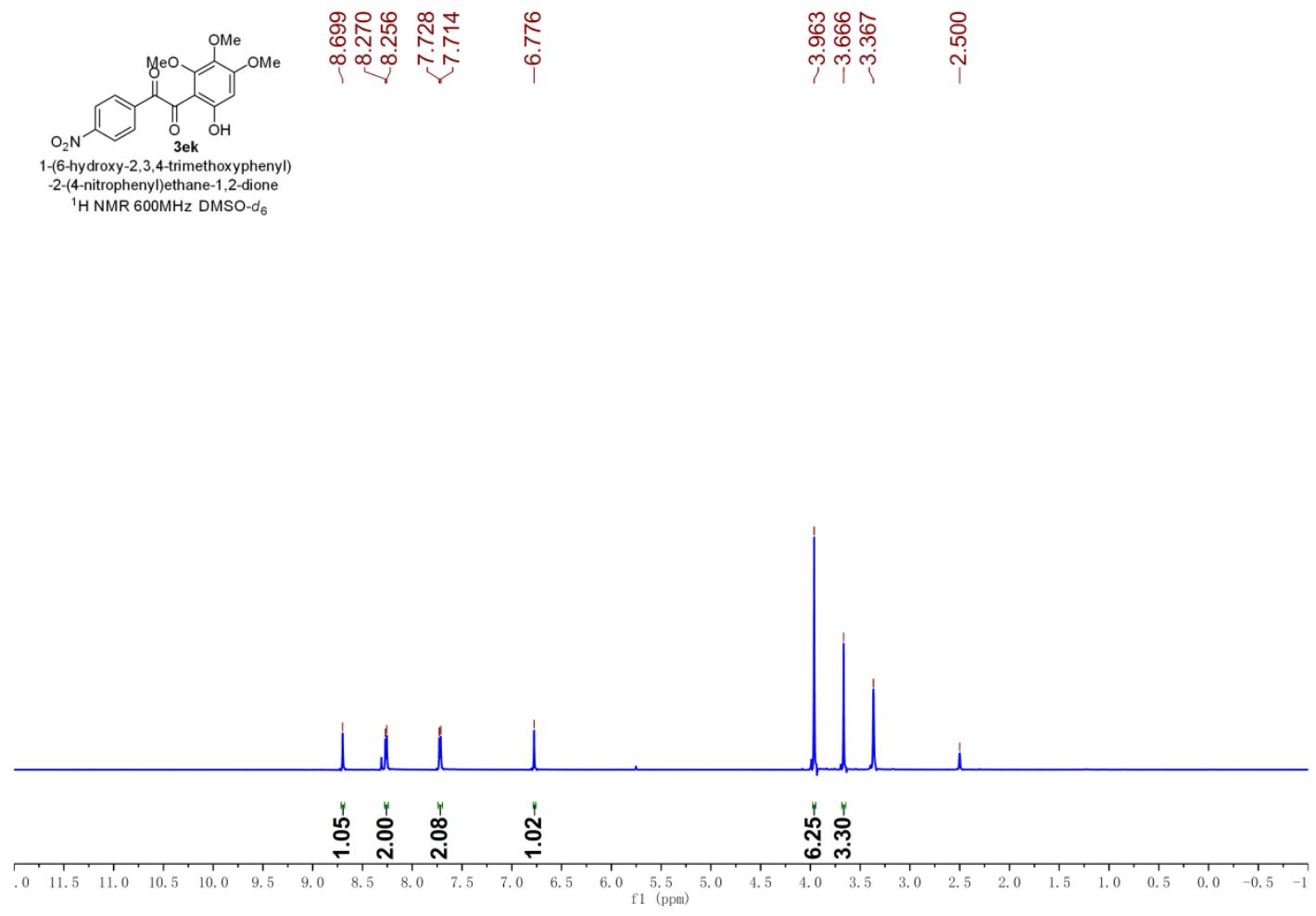

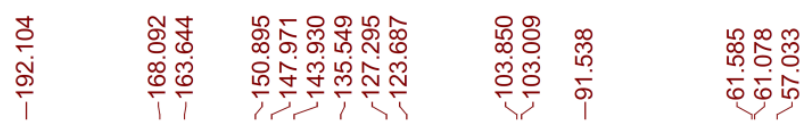

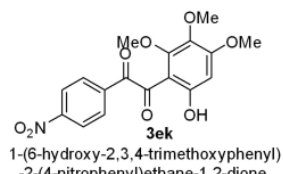

1-(6-hydroxy-2,3,4-trimethoxypheny

${ }^{13} \mathrm{C}$ NMR $150 \mathrm{MHz}$ DMSO-d $d_{6}$

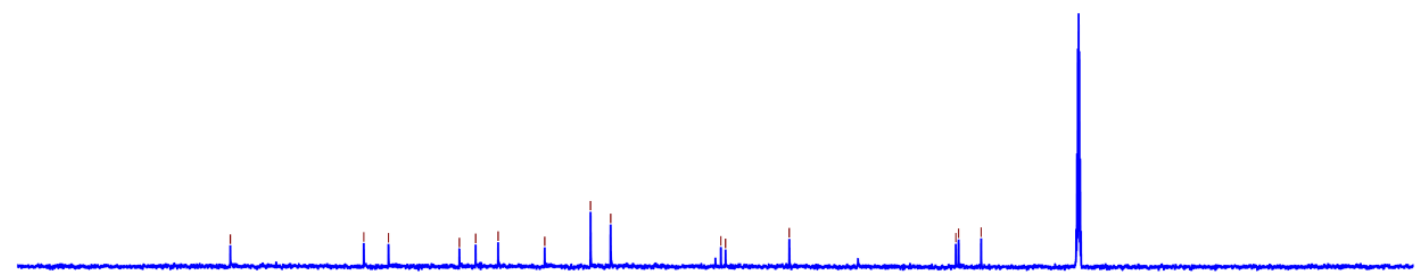

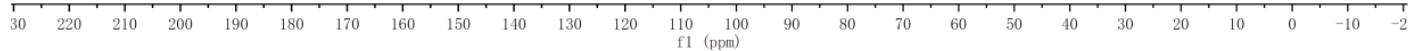




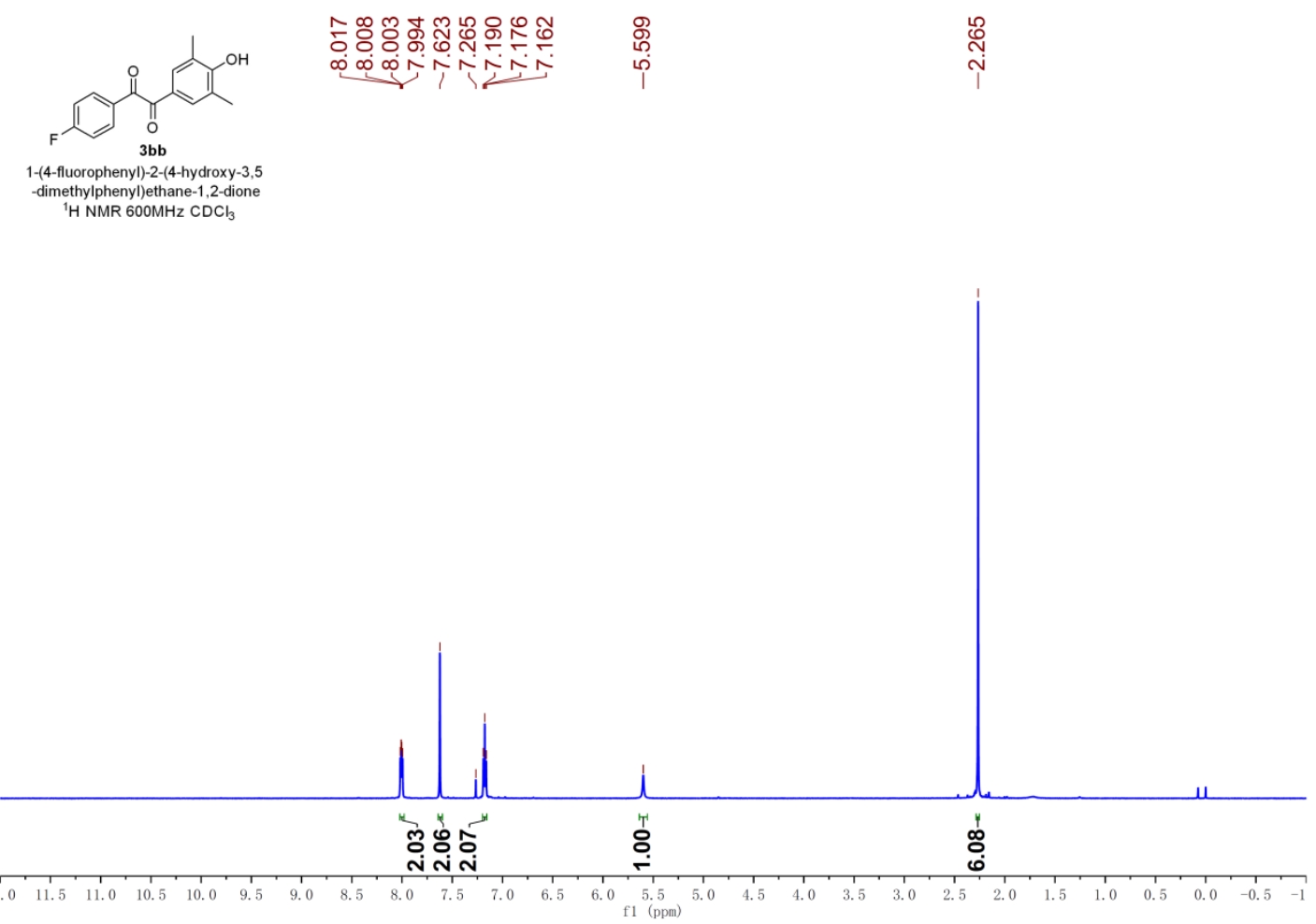

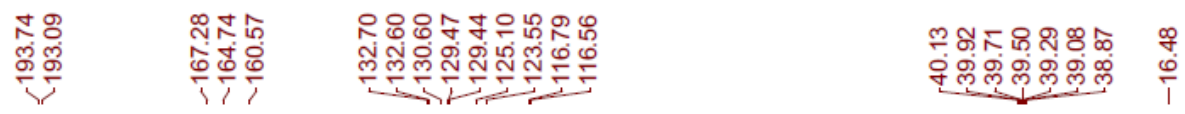

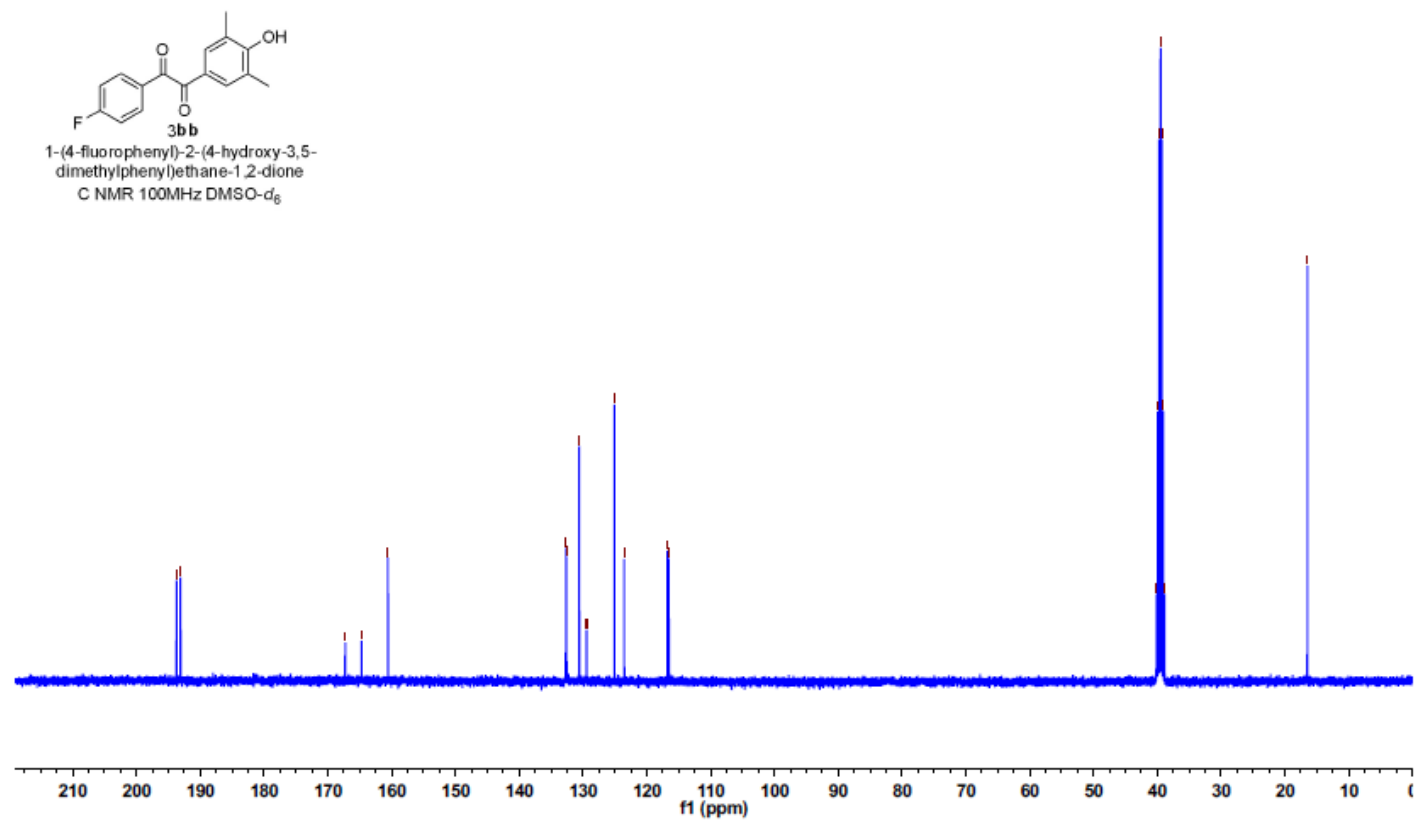



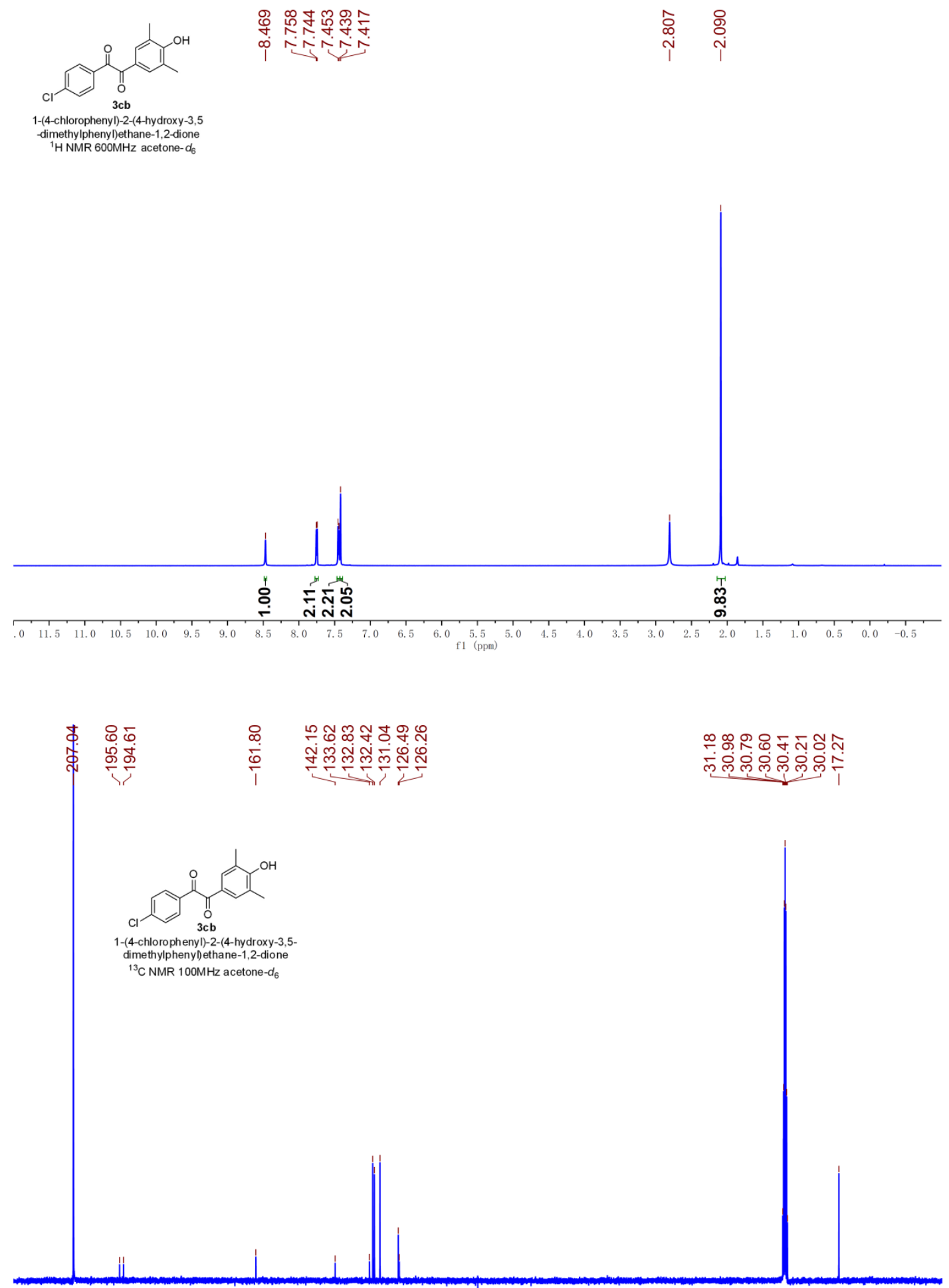

$\begin{array}{llllllllllllllllllllllllllllll}220 & 210 & 200 & 190 & 180 & 170 & 160 & 150 & 140 & 130 & 120 & \begin{array}{l}110 \\ \mathrm{f}(\mathrm{ppm})\end{array} & 100 & 90 & 80 & 70 & 60 & 50 & 40 & 30 & 20 & 10 & 0\end{array}$ 


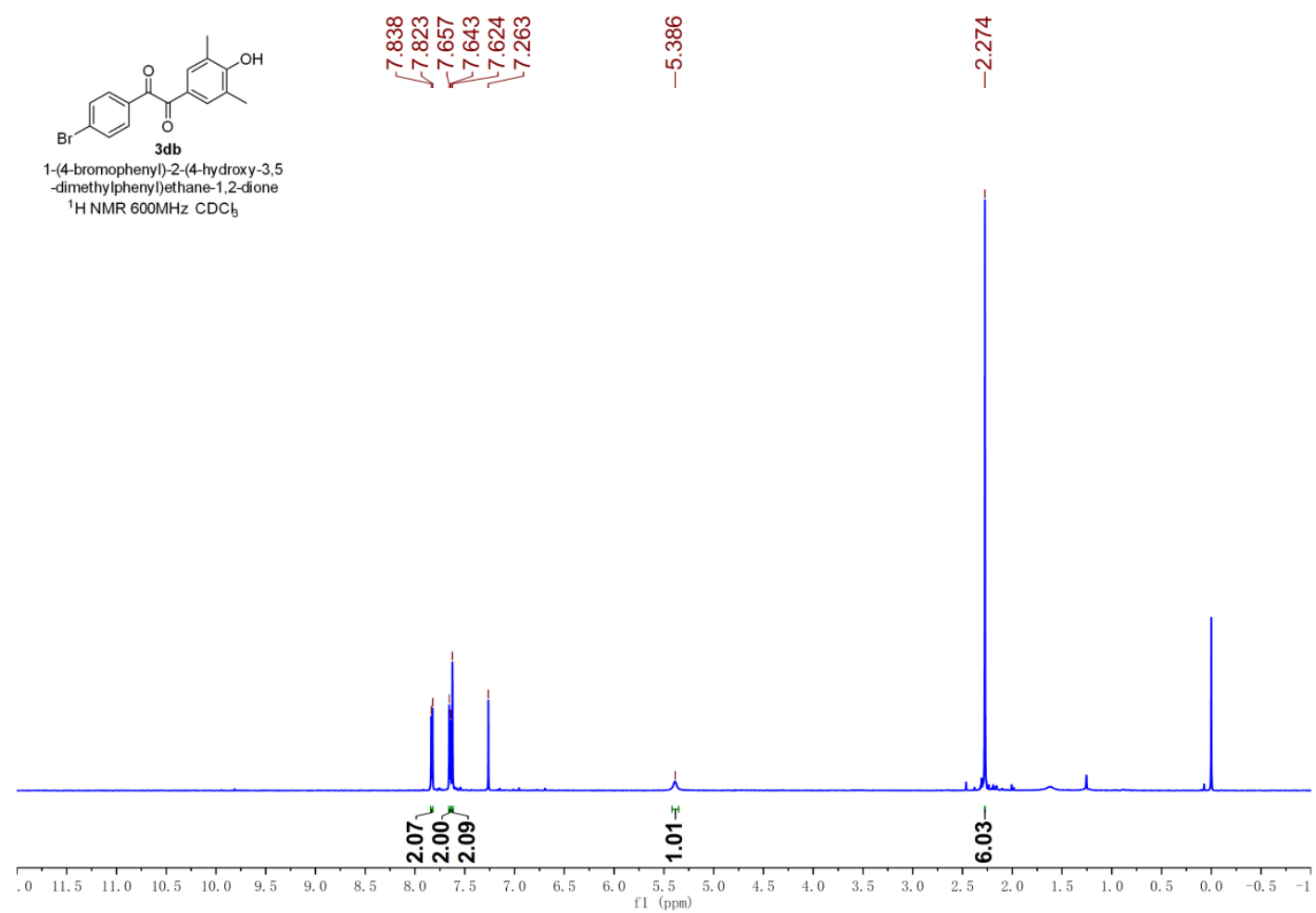

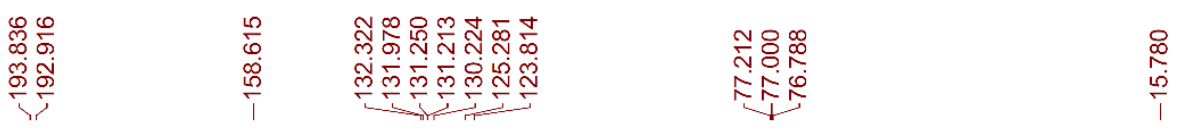

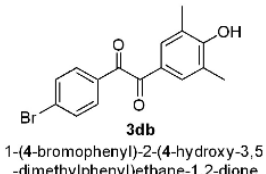

-dimethylph

${ }^{13} \mathrm{C}$ NMR $150 \mathrm{MHz} \mathrm{CDCb}_{3}$

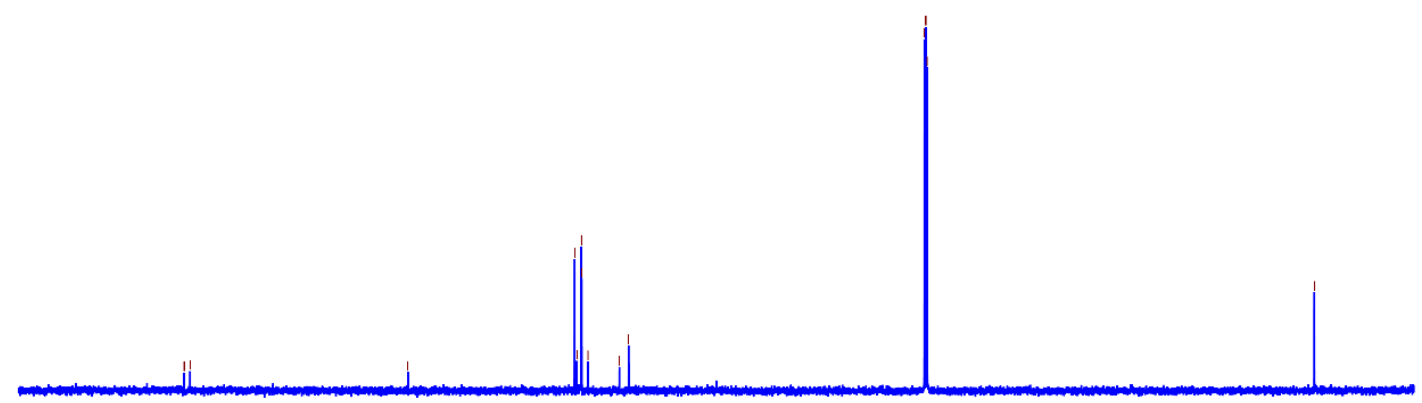

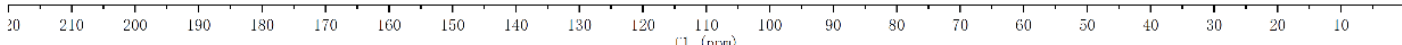




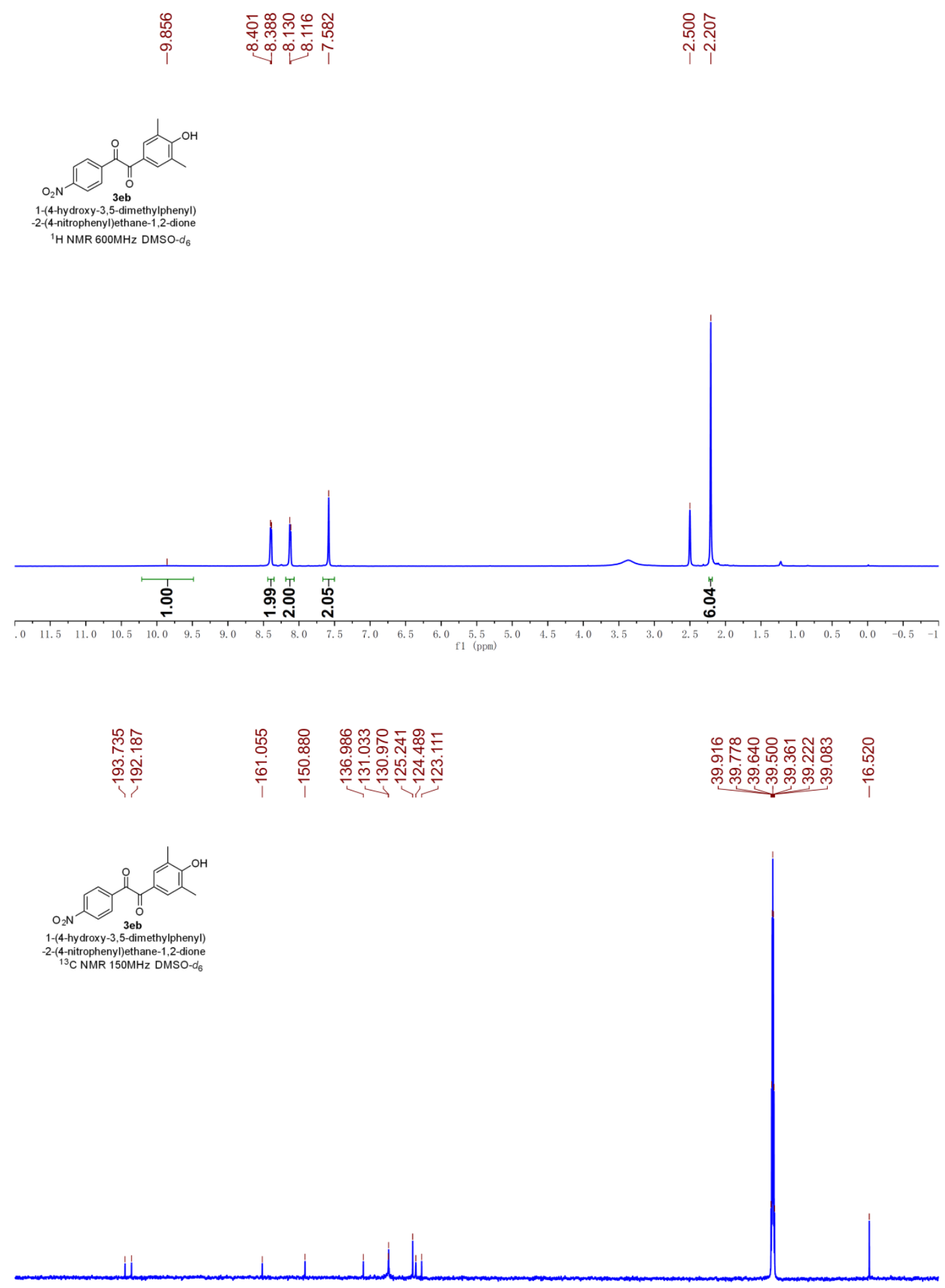

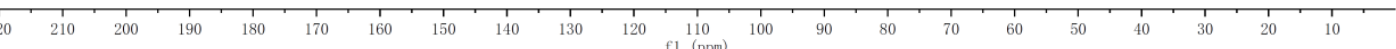




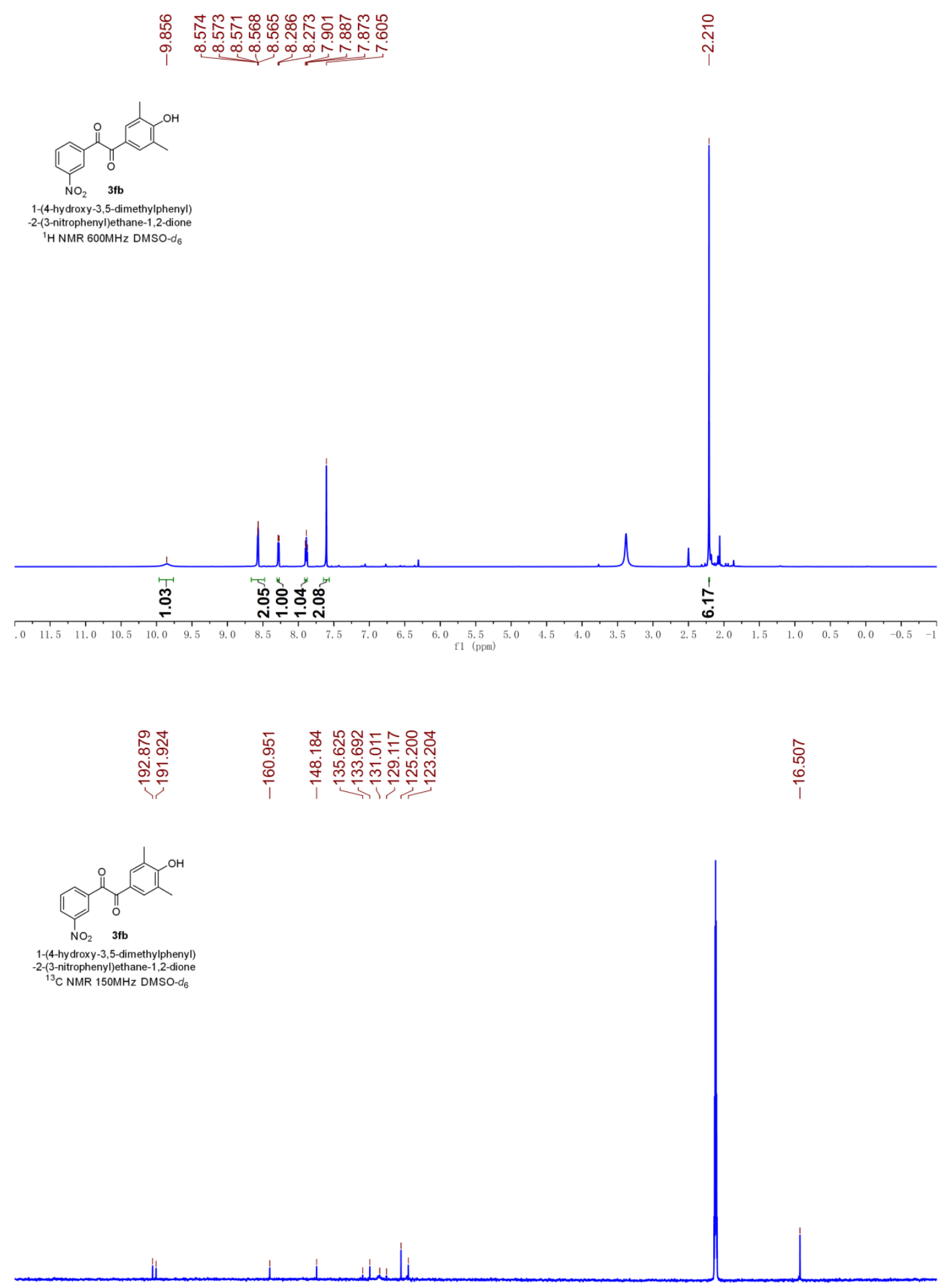

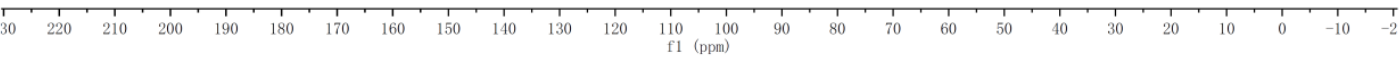



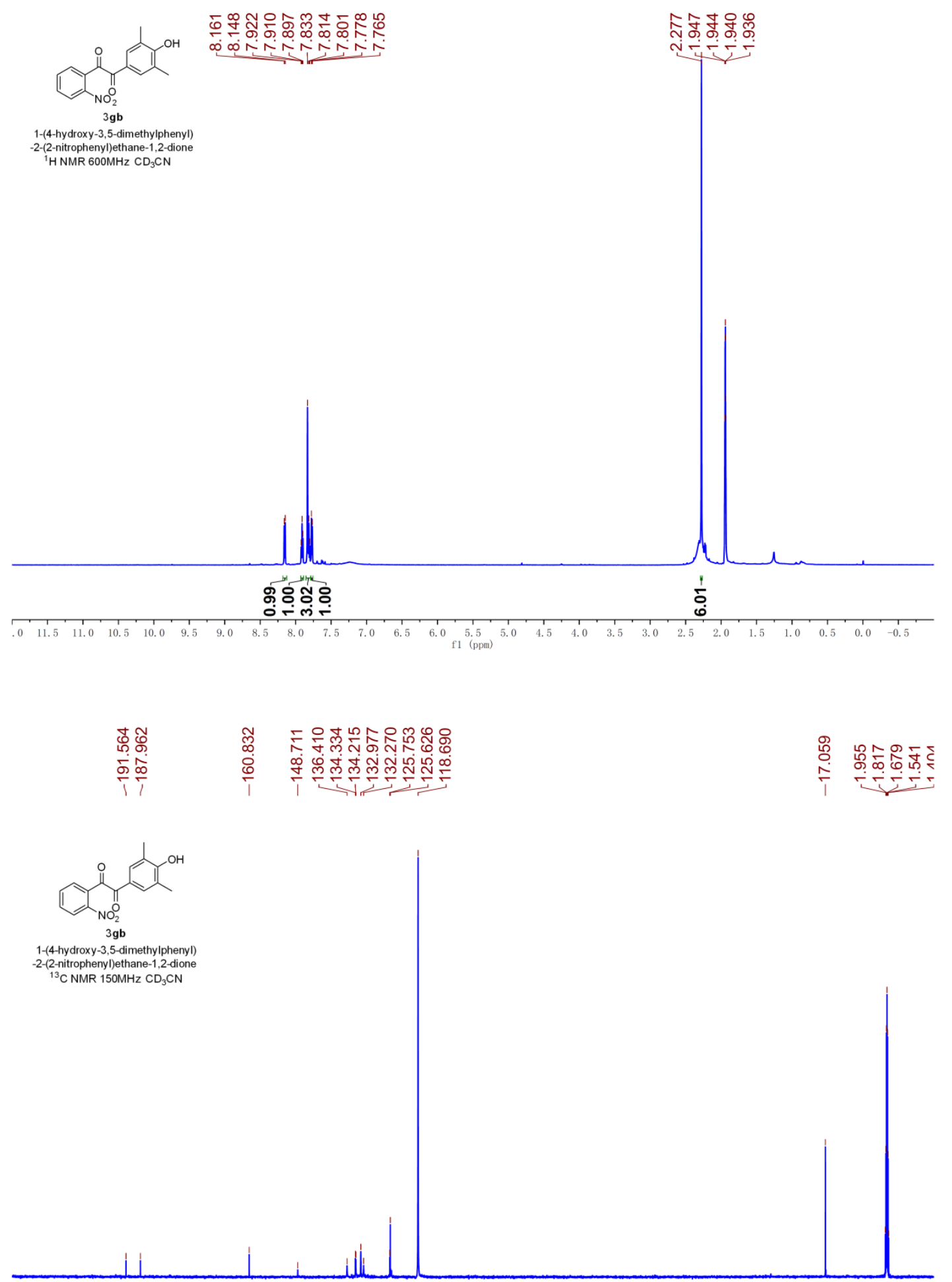

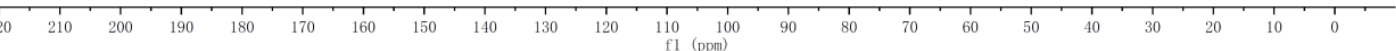



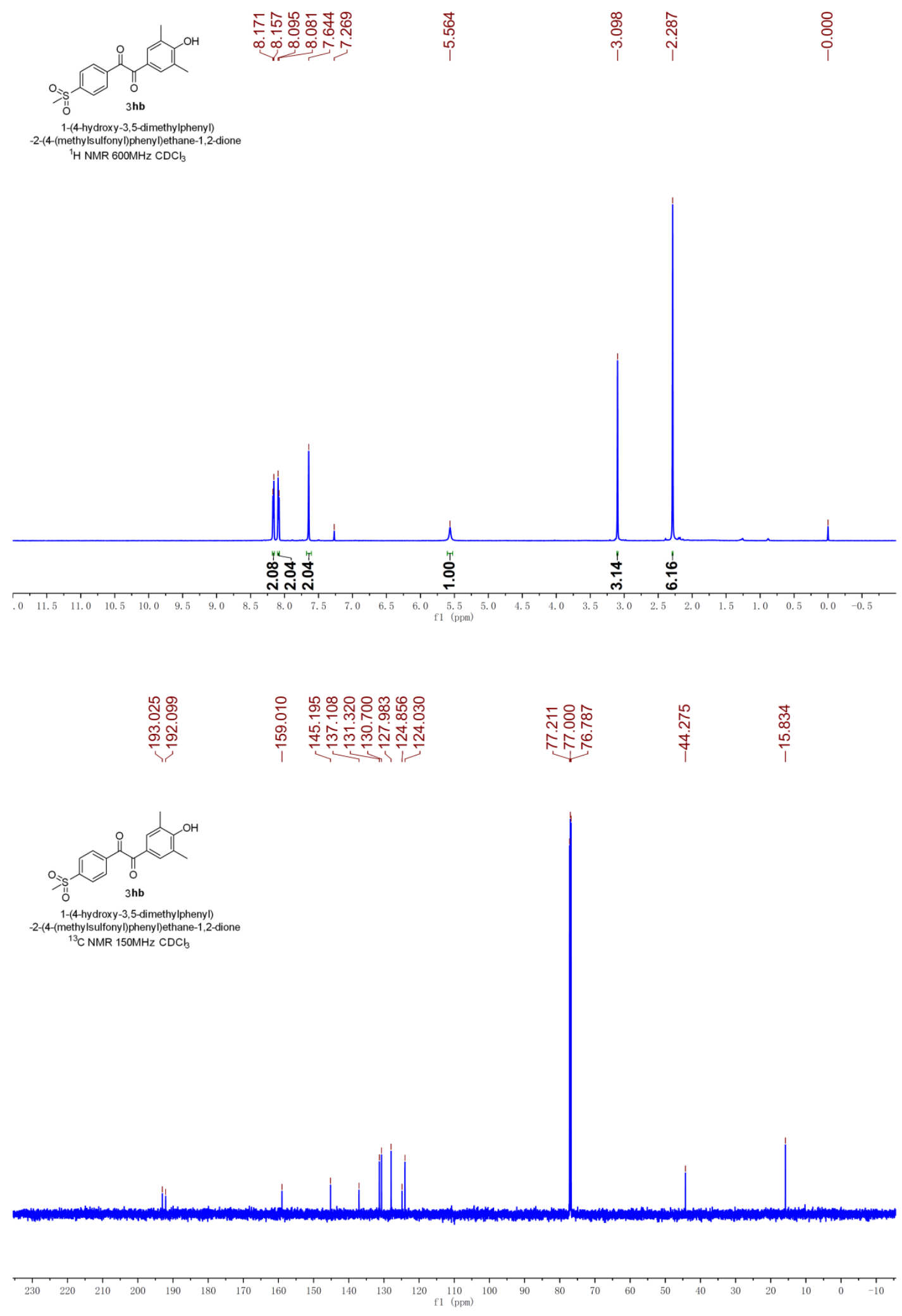


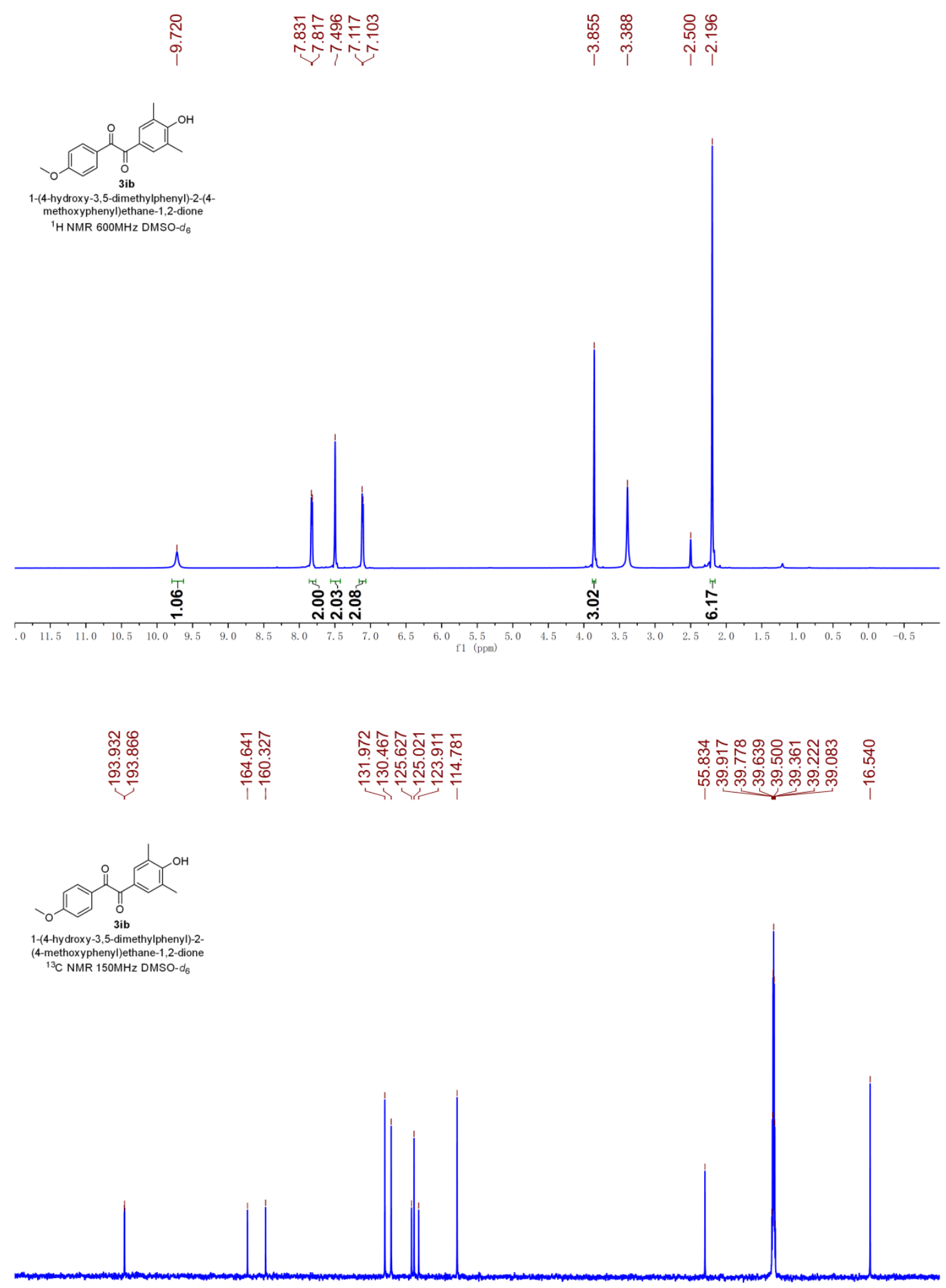

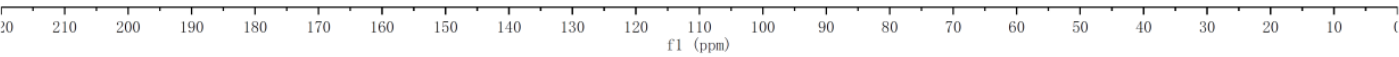




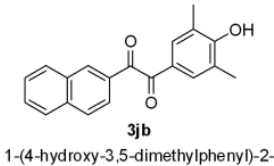

1-(4-hydroxy-3,5-dimethylphenyl)-2-

${ }^{1} \mathrm{H}$ NMR $400 \mathrm{MHz}$ DMSO- $d_{6}$
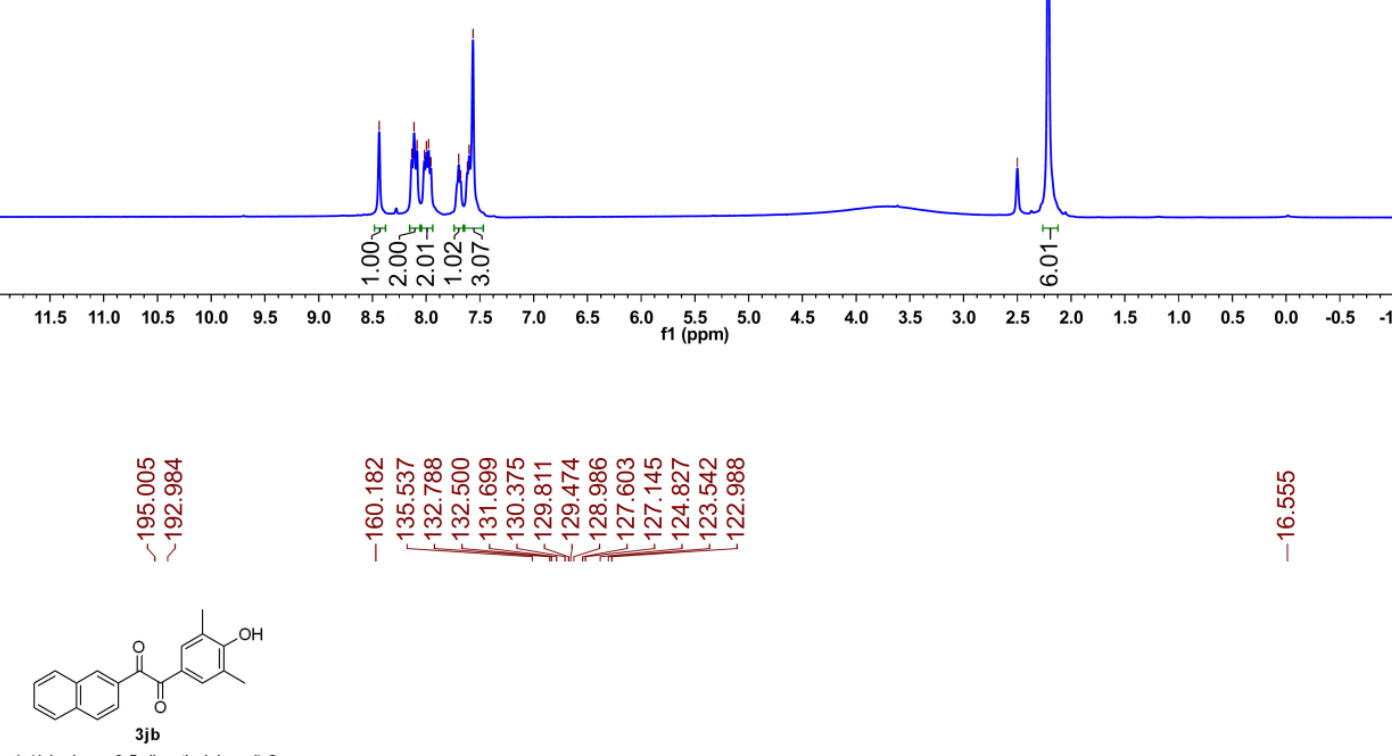

1-(4-hydroxy-3,5-dimethy lphenyl)-2-

(naphthalen-2-y) ethane-1,2

${ }^{33} \mathrm{C}$ NMR $100 \mathrm{MHz}$ DMSO-d

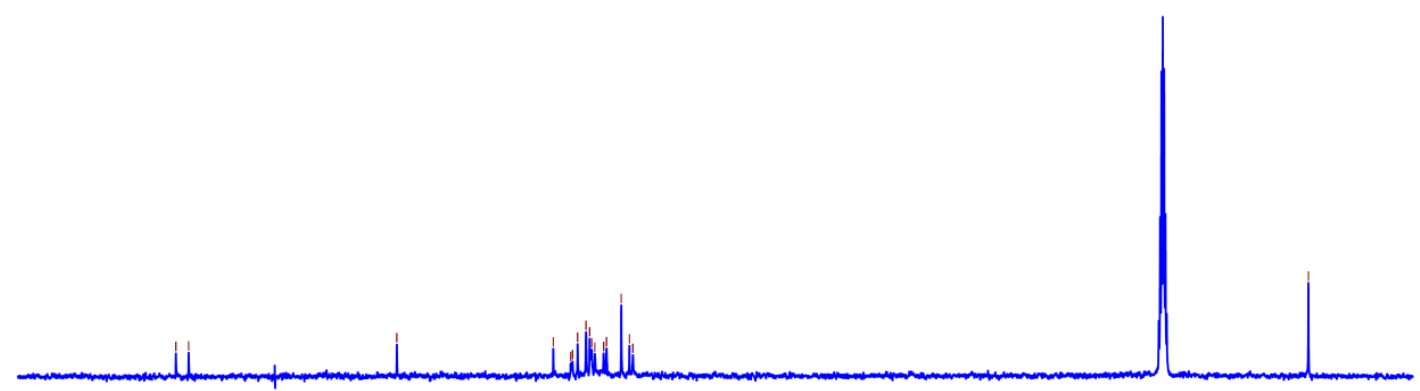

$\begin{array}{llllllllllllllllllllllll}20 & 210 & 200 & 190 & 180 & 170 & 160 & 150 & 140 & 130 & 120 & 110 & 100 & 90 & 80 & 70 & 60 & 50 & 40 & 30 & 20 & 10 & 10\end{array}$ 

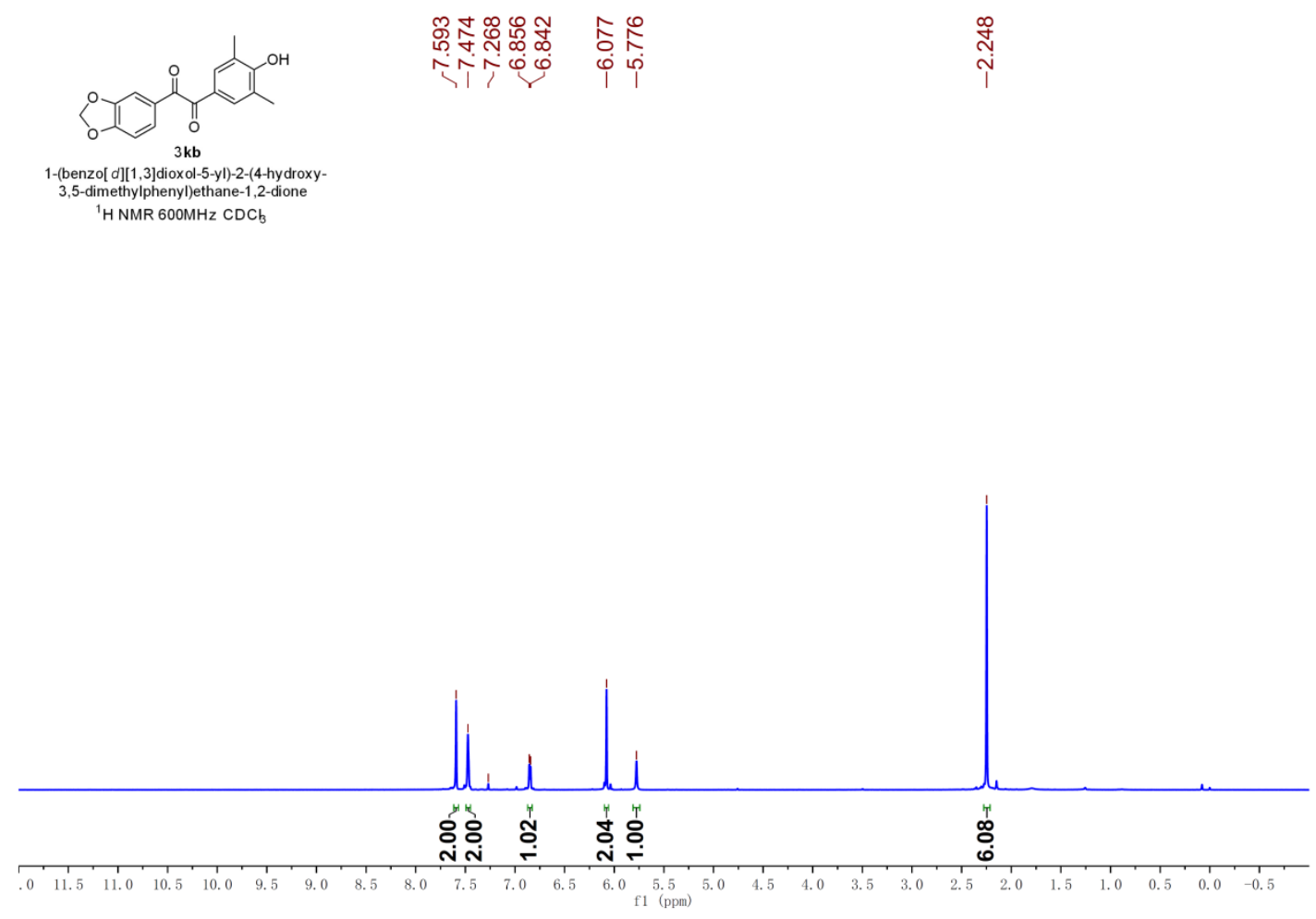

余㶽

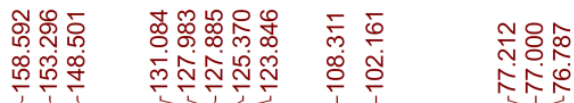

$\frac{10}{\infty}$
10
$\frac{10}{1}$

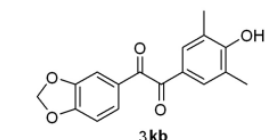

1-(benzo[ of [1,3]dioxol-5-yl)-2-(4-hydroxy-

,5-dimethylphenyl)ethane-1,2-dio

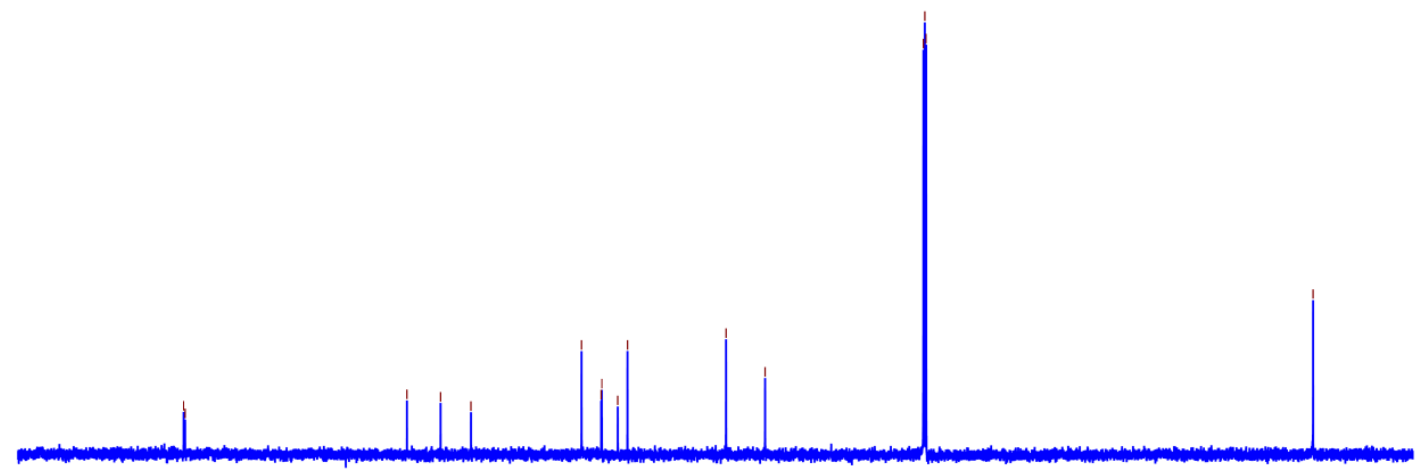

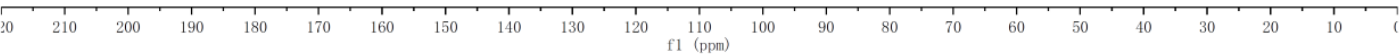



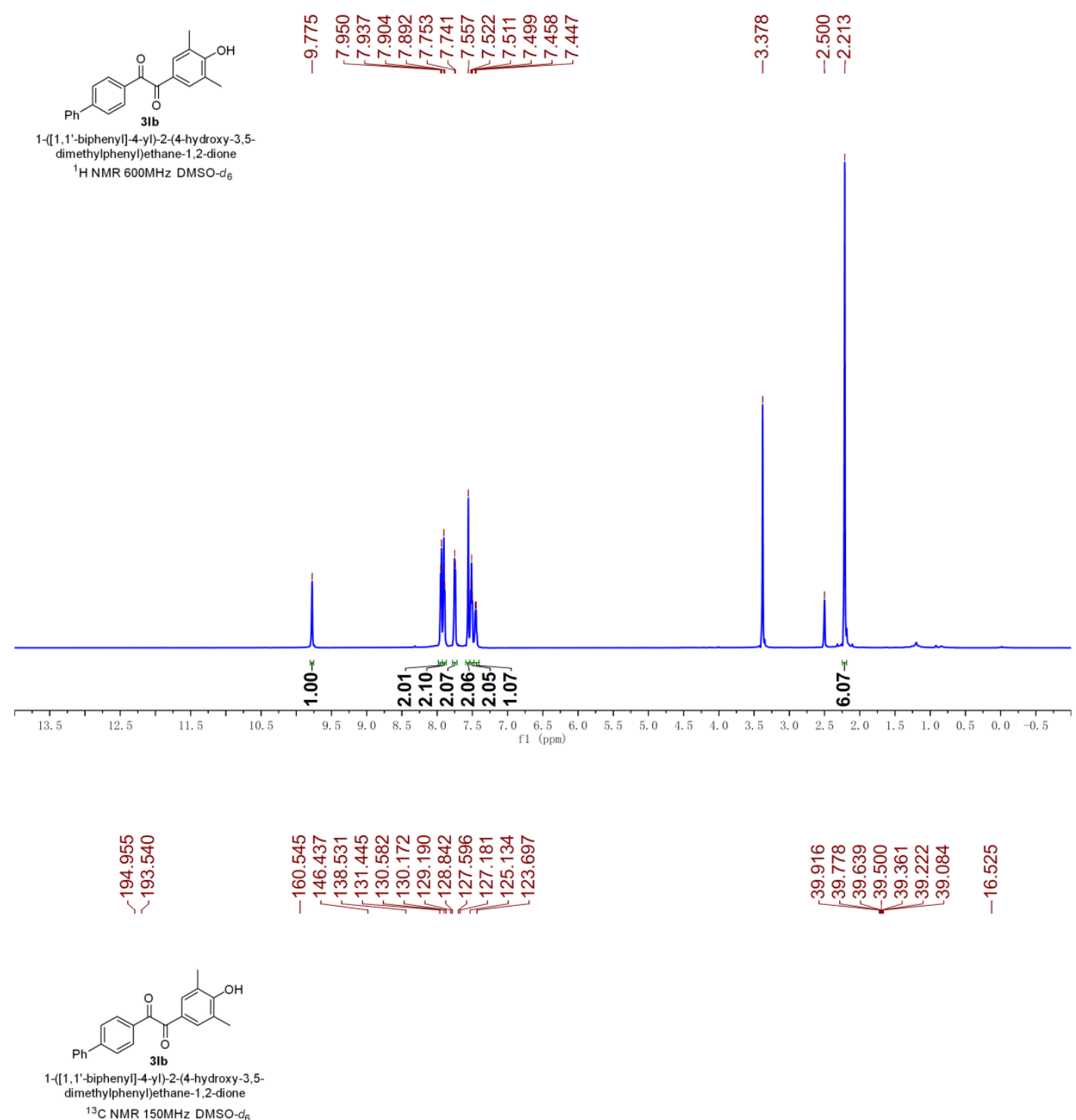

${ }^{13} \mathrm{C}$ NMR $150 \mathrm{MHz}$ DMSO-d
6

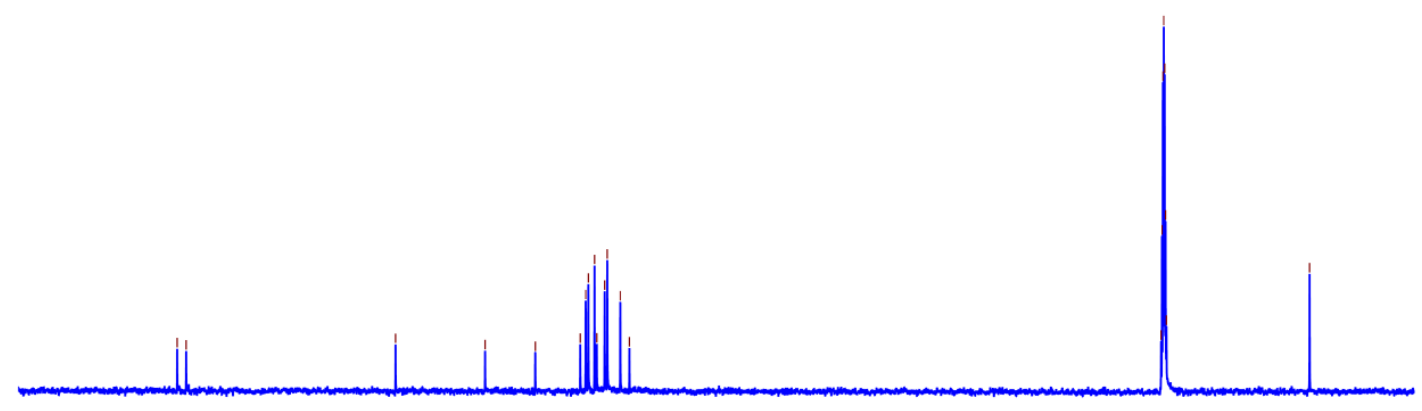

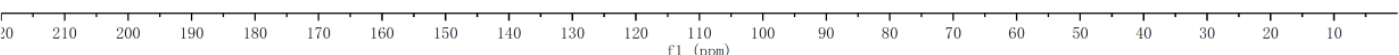




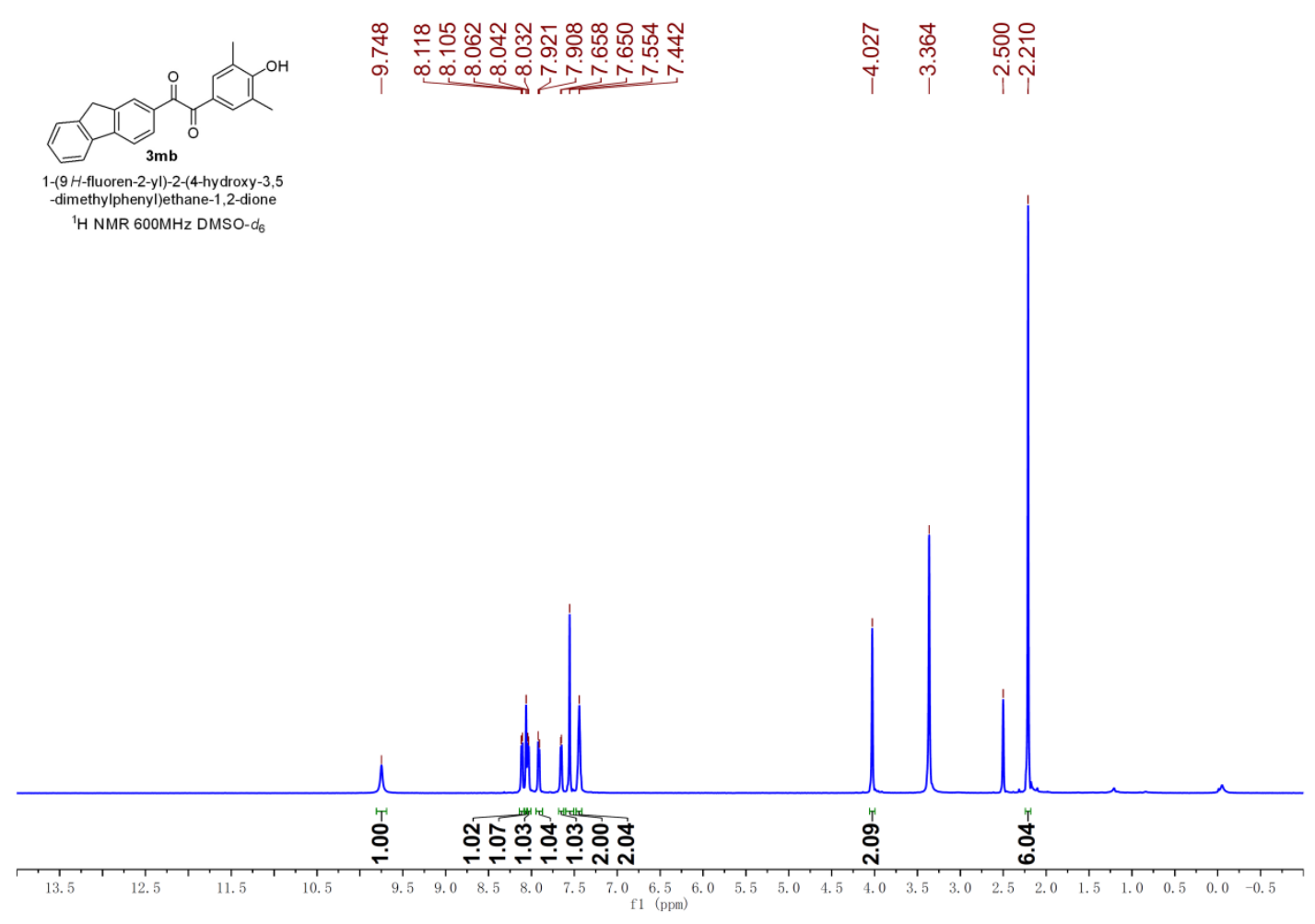

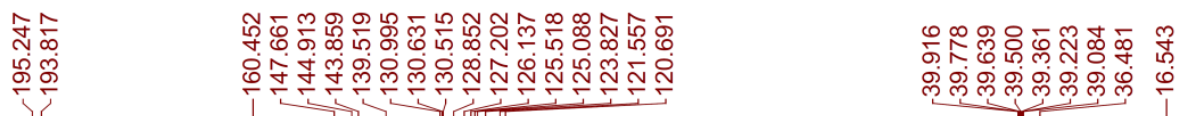

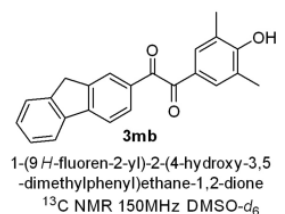

${ }^{13} \mathrm{C}$ NMR $150 \mathrm{MHz}$ DMSO-d 


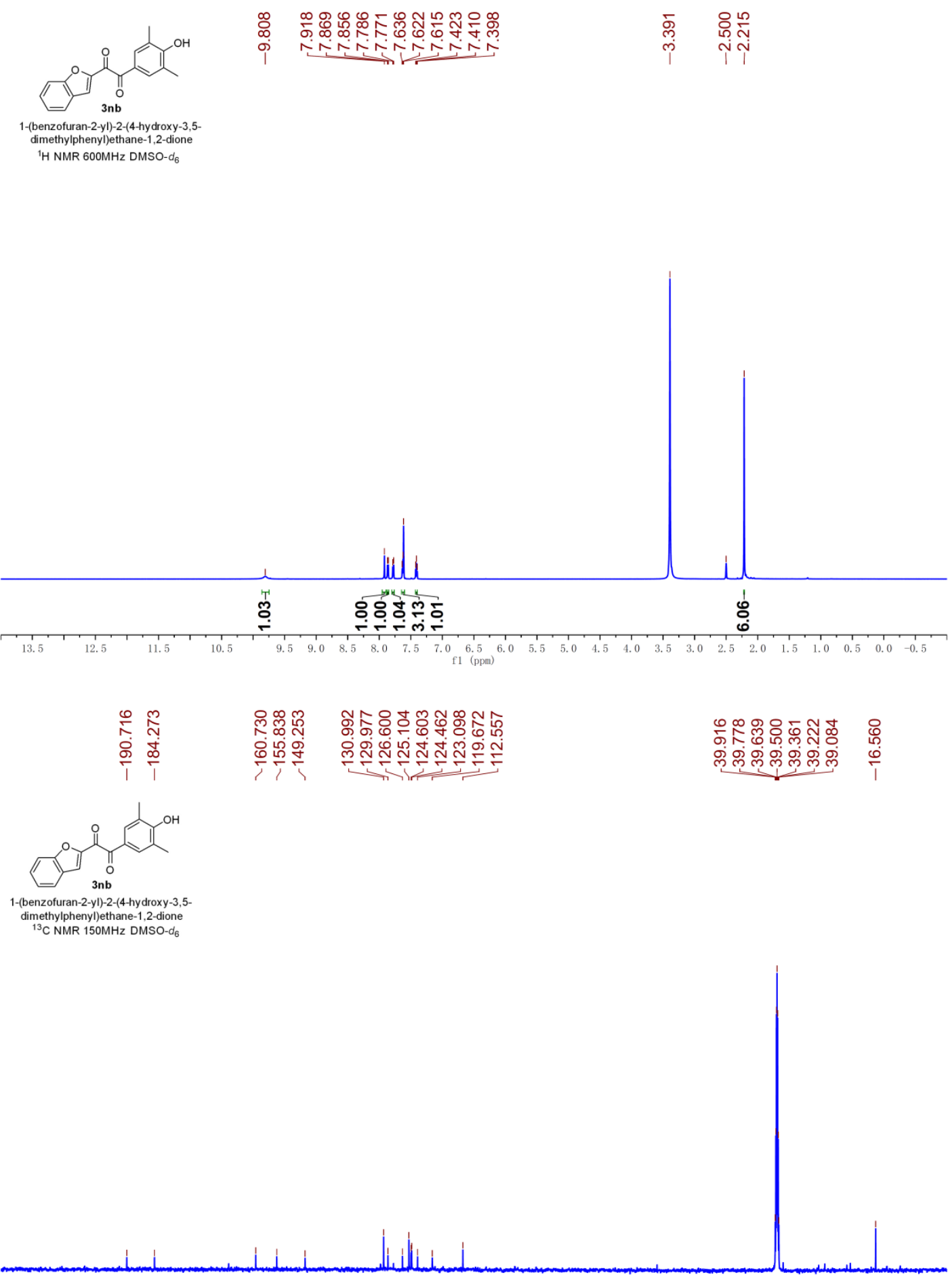

13.-(benzofuran-2-yl)-2-(4-hydroxy-3,5-

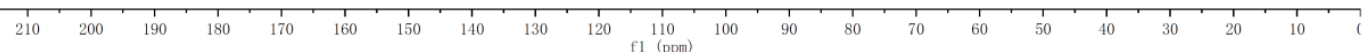



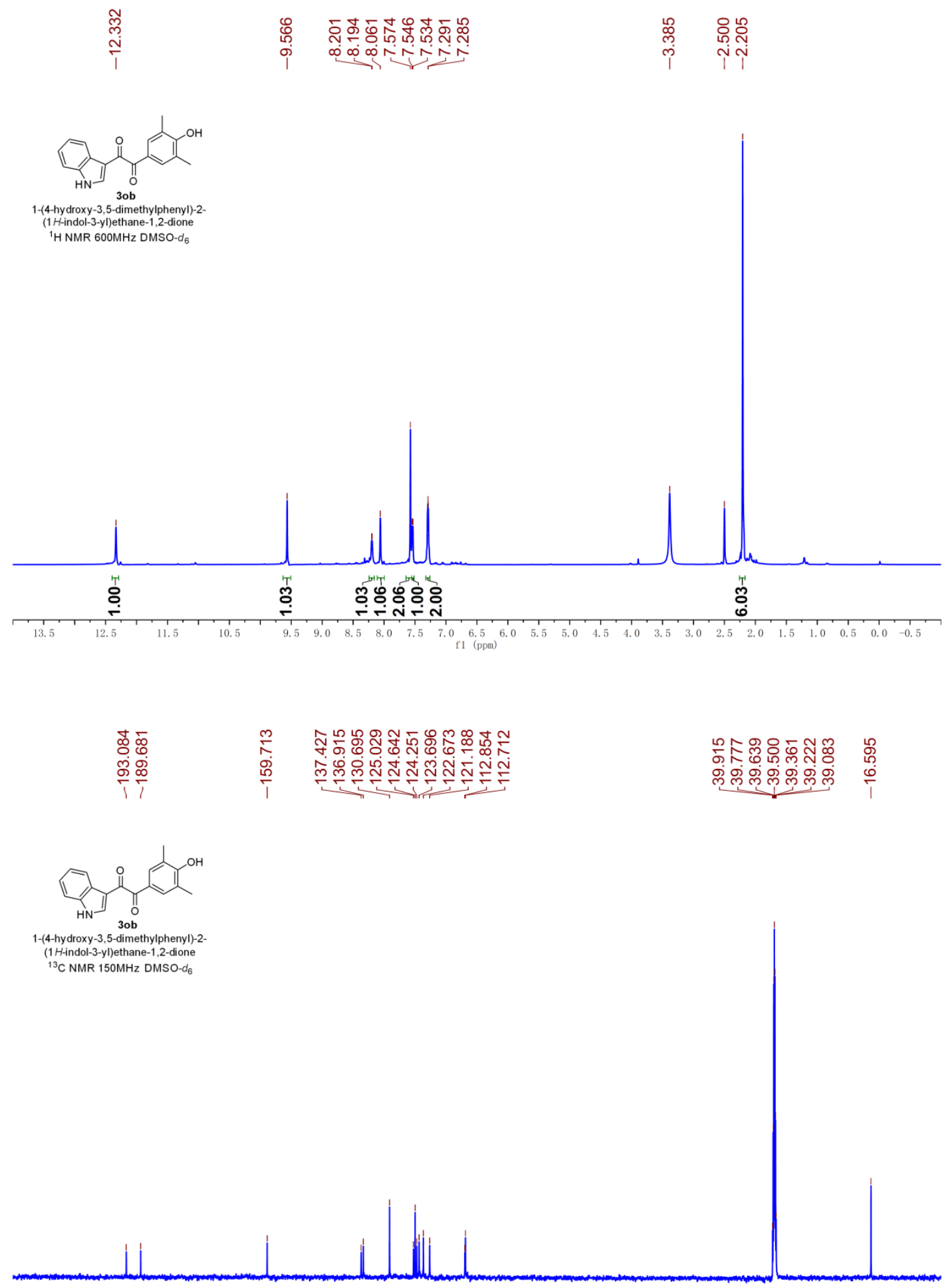

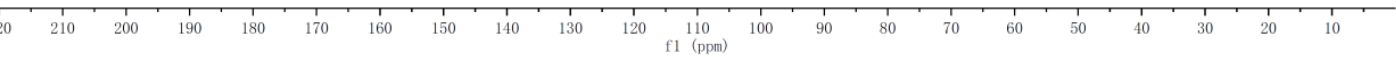




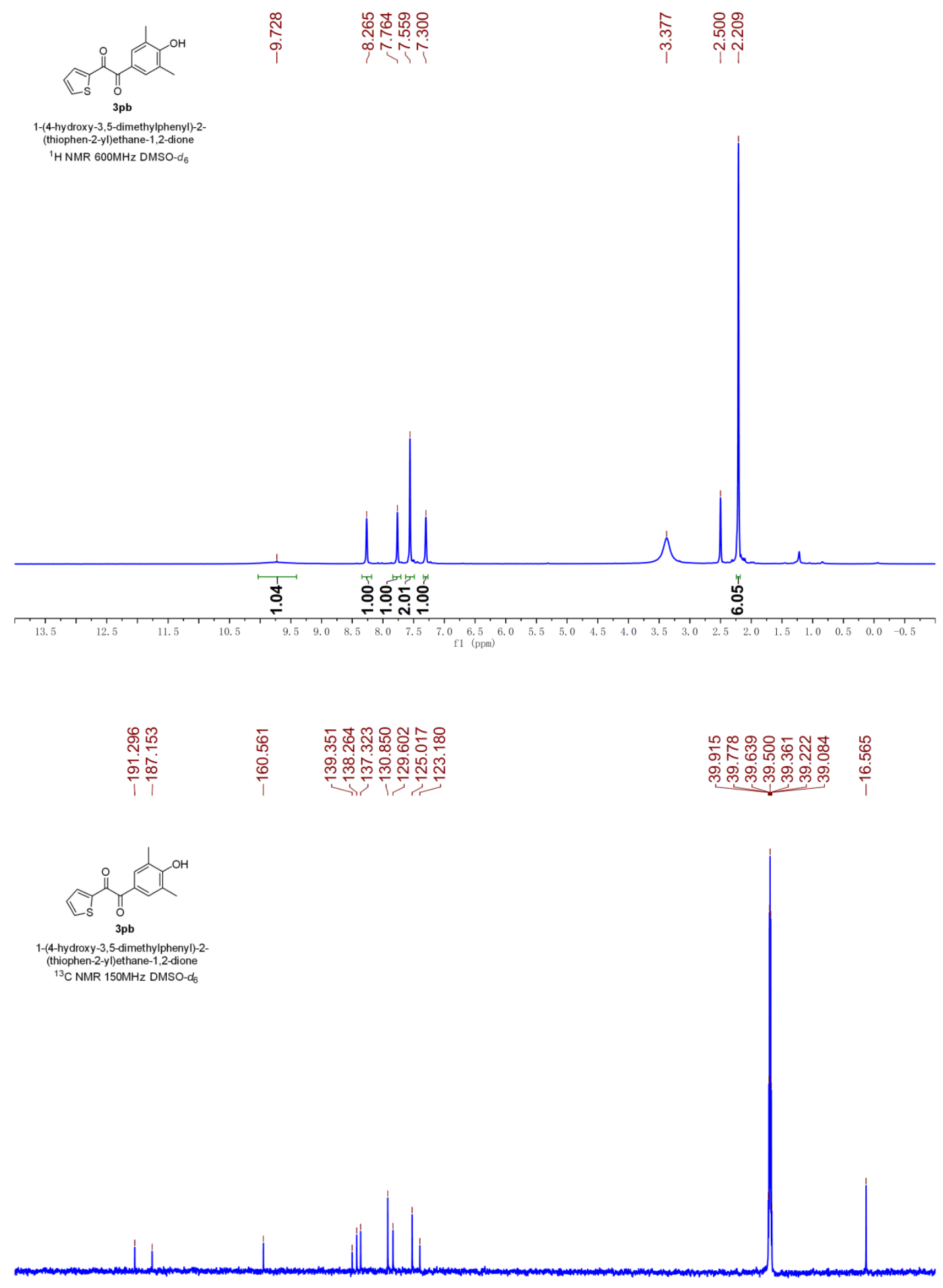

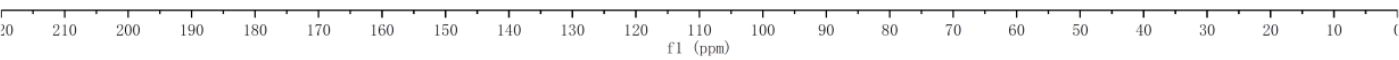




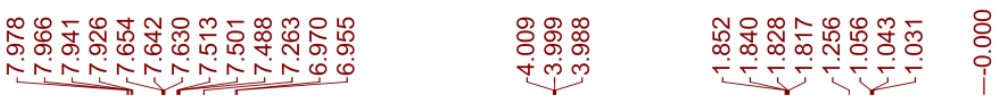

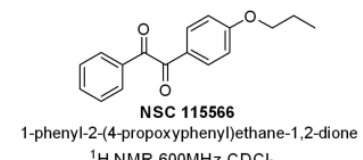

${ }^{1} \mathrm{HNMR} 600 \mathrm{MHz} \mathrm{CDCl}$

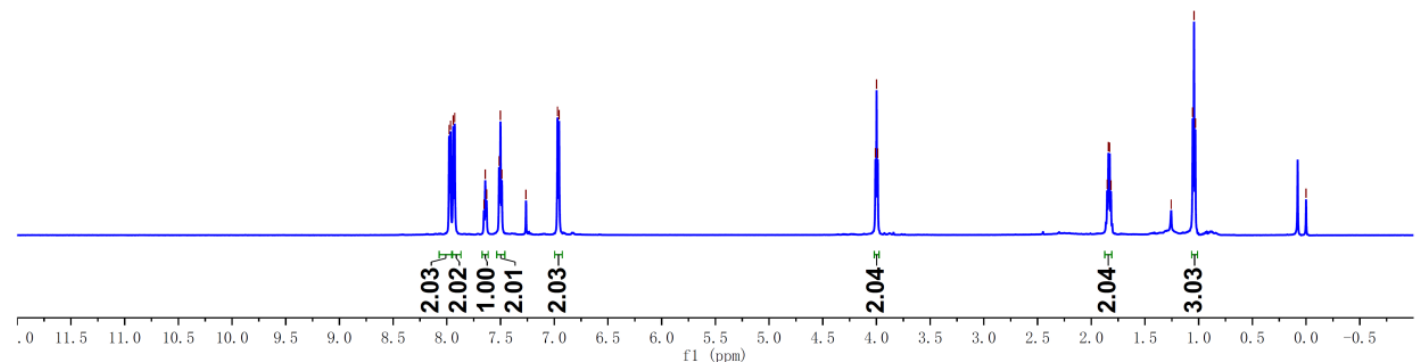

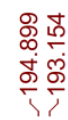

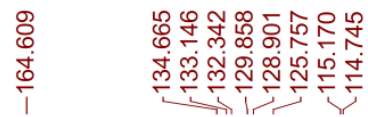

సุำ

쇼융ㅇㅇ

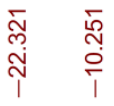

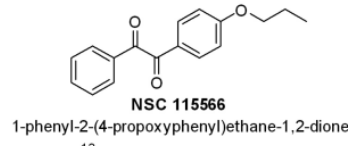

${ }^{13} \mathrm{C}$ NMR $150 \mathrm{MHz} \mathrm{CDCb}$

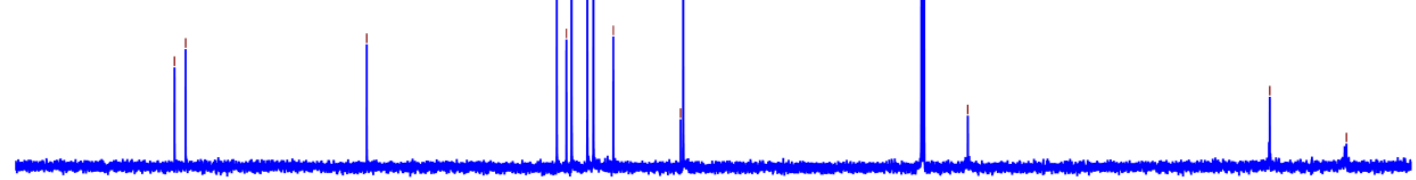

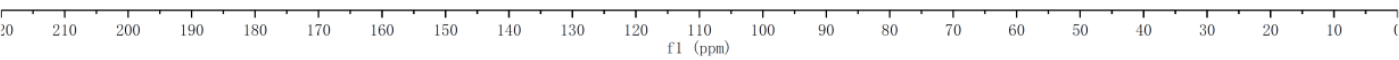



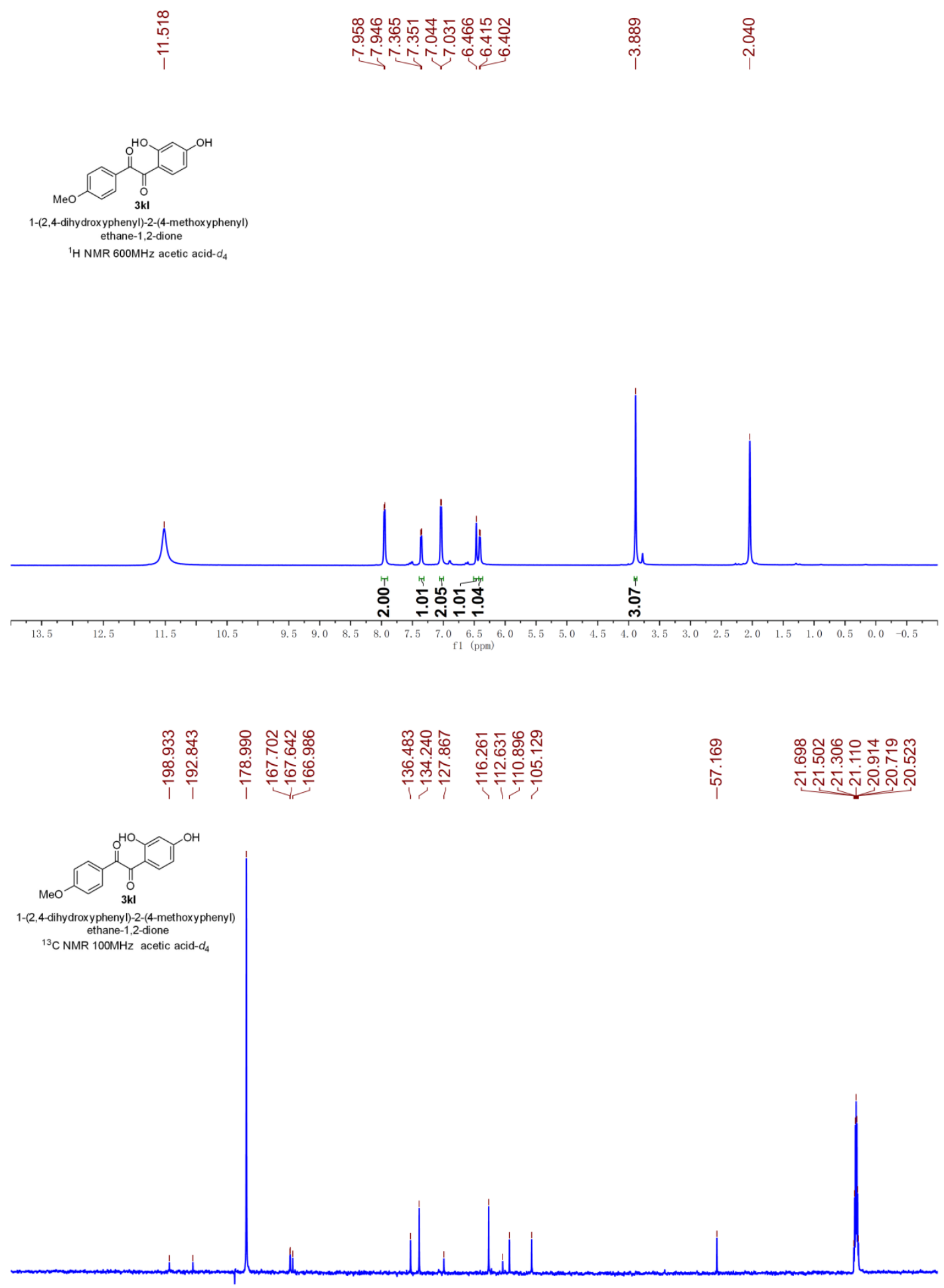

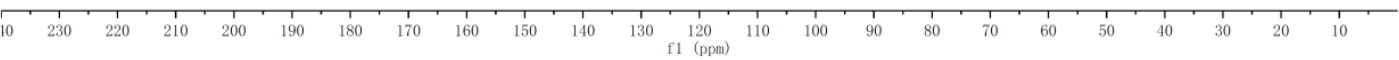



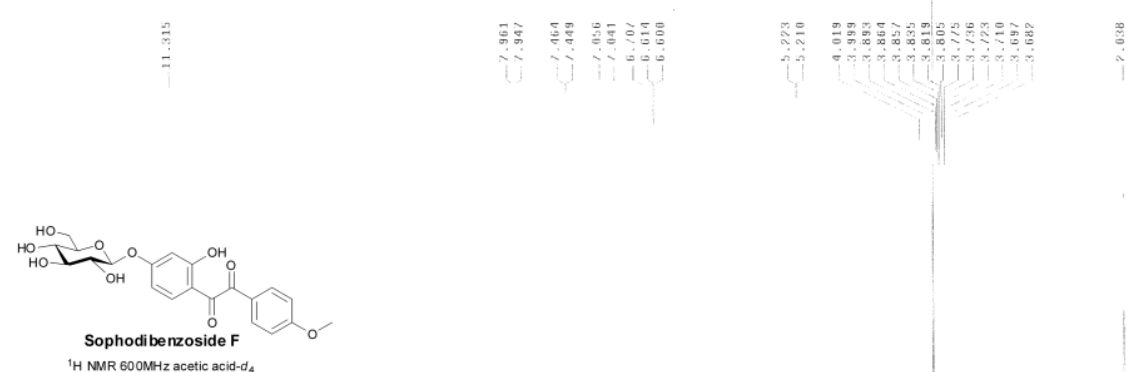

H NMR 60OMHz acetic acid-dod

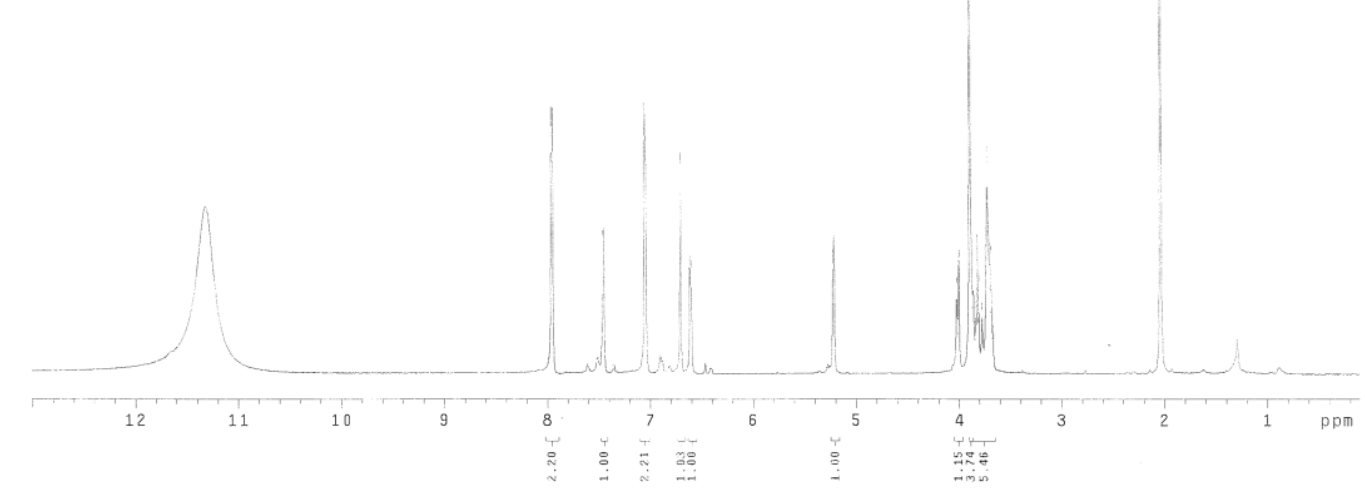

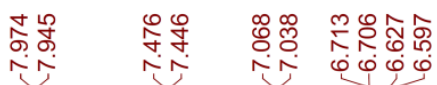

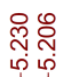

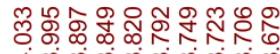

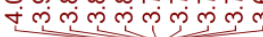
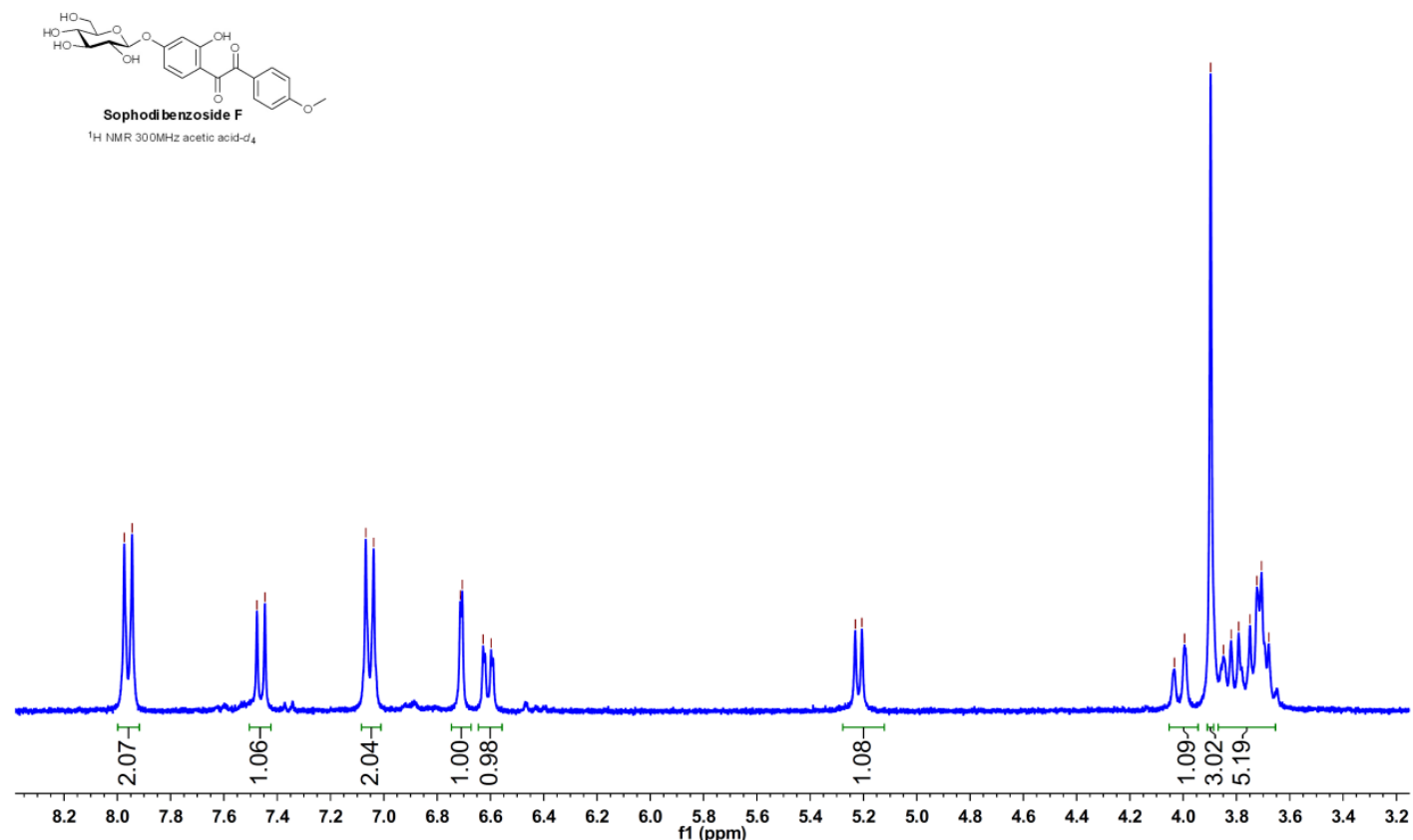

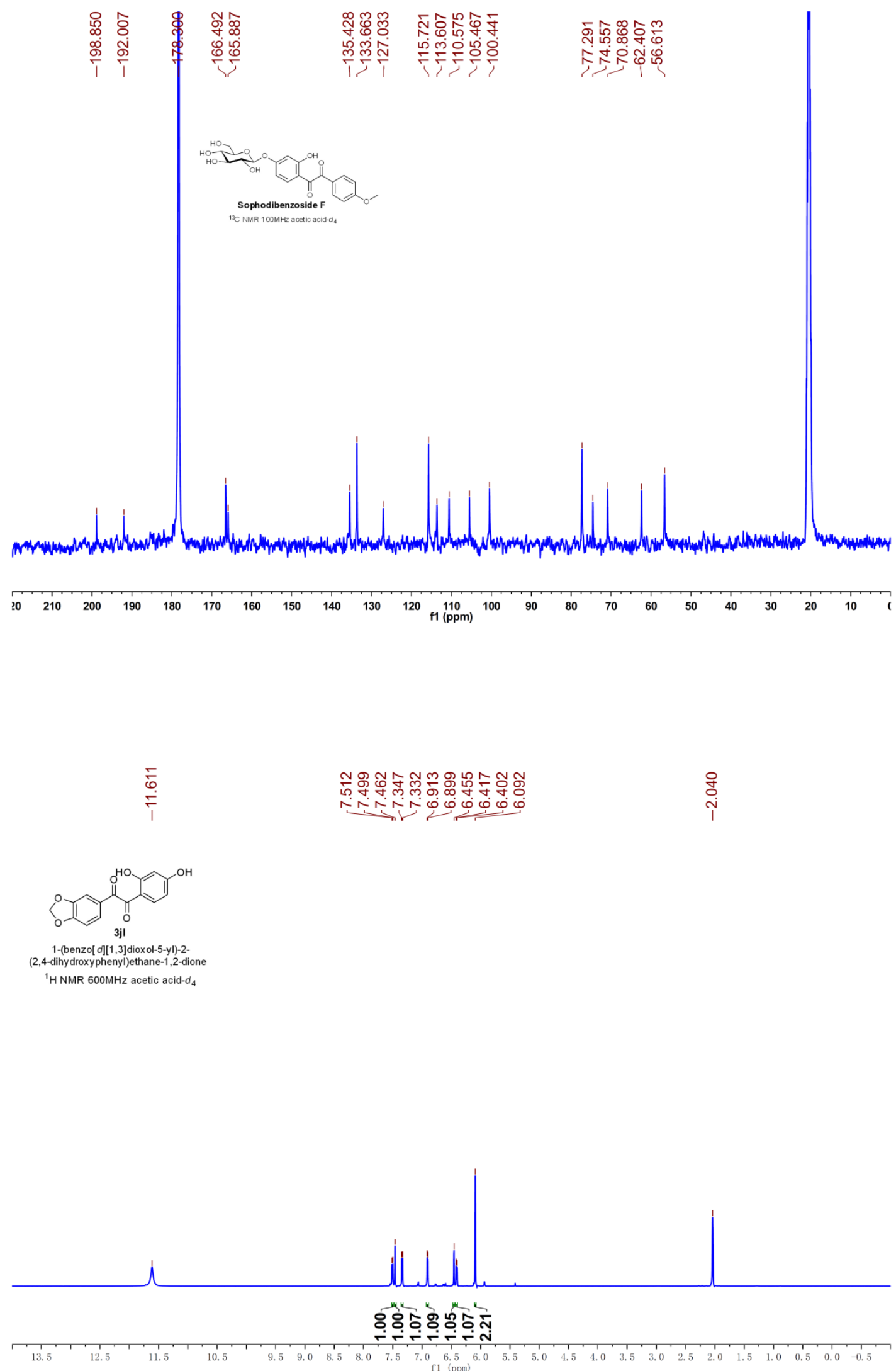


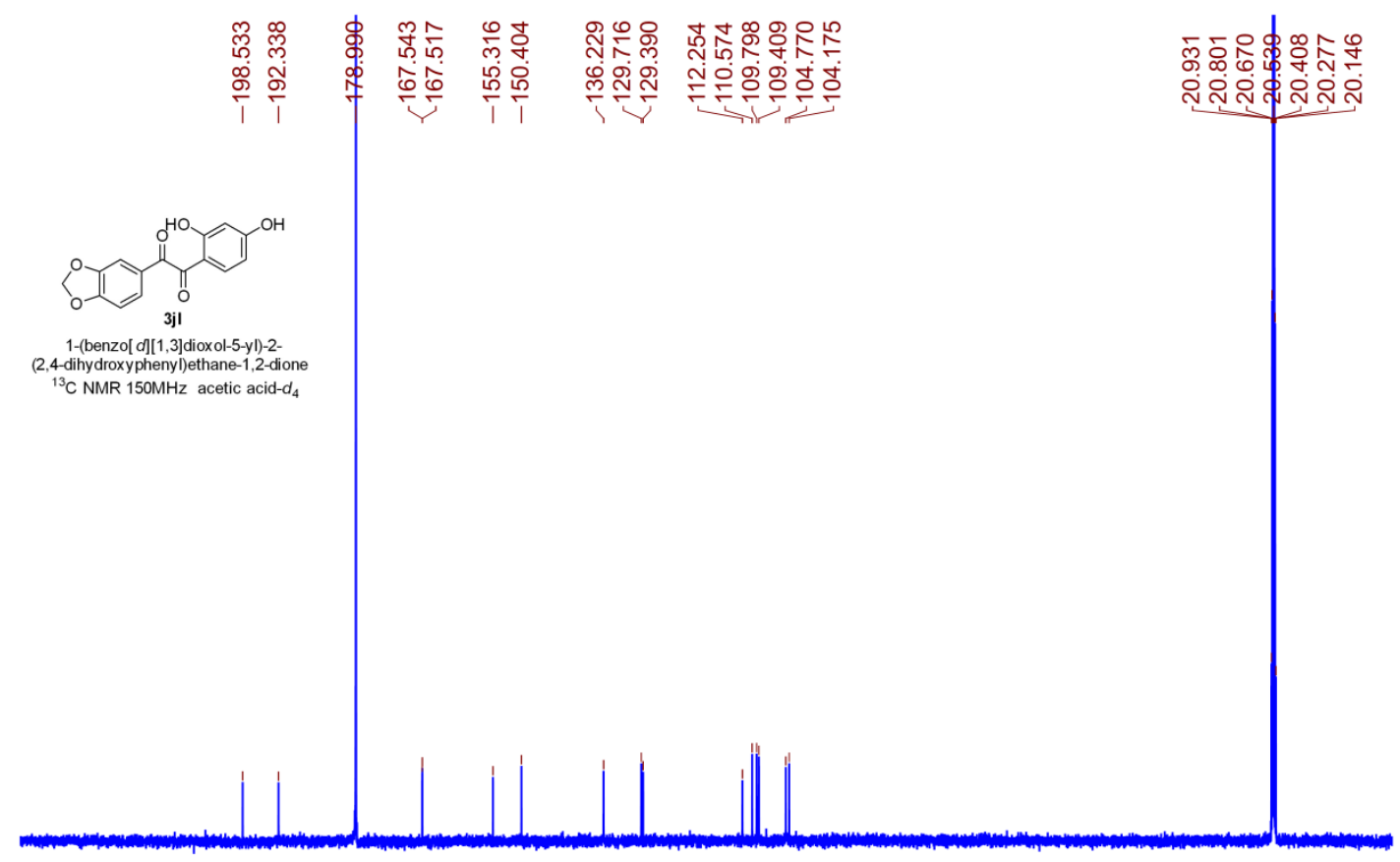

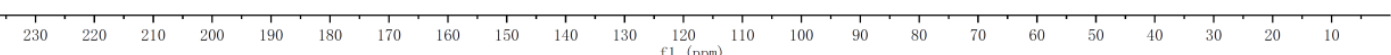

Saapp 1e: xj̉c1 väg-t

Solvent: aceticacid
Date: May 6 : 2016

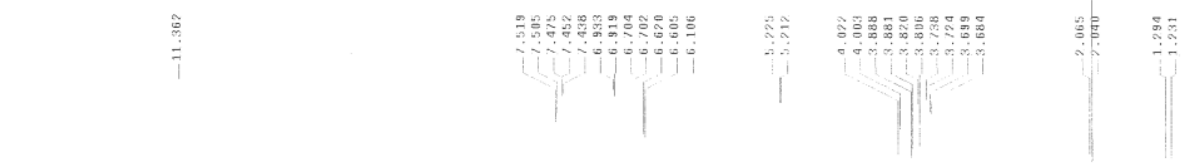

о он

Sophodibenzoside I

IH NMR $600 \mathrm{NH}$ z acetic acid-d

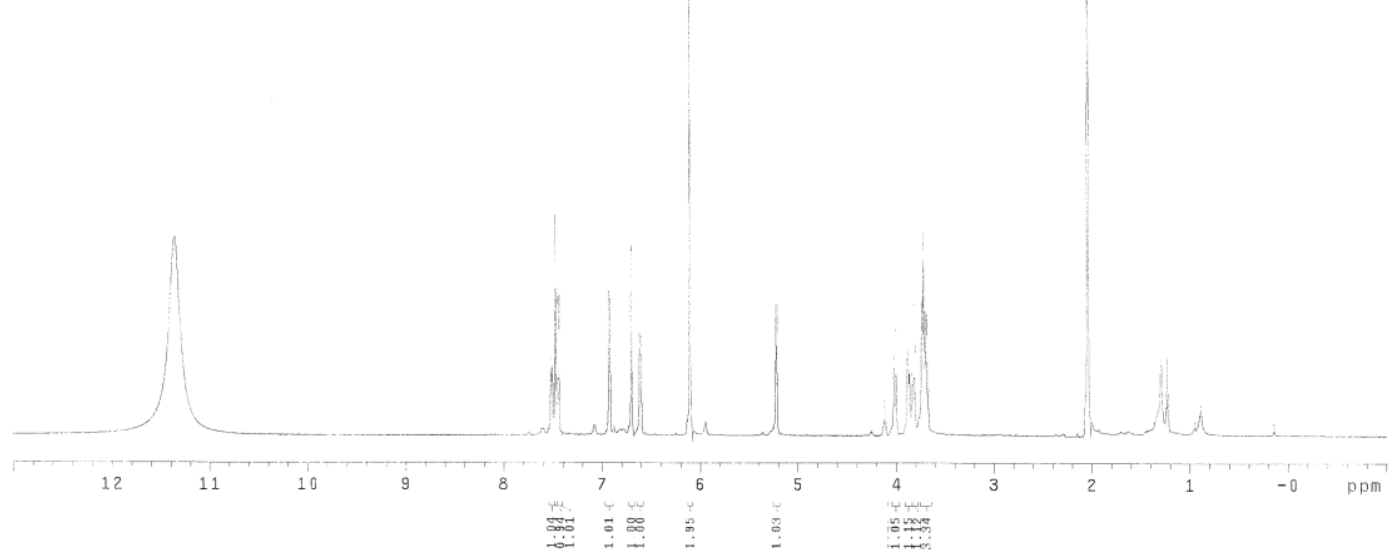



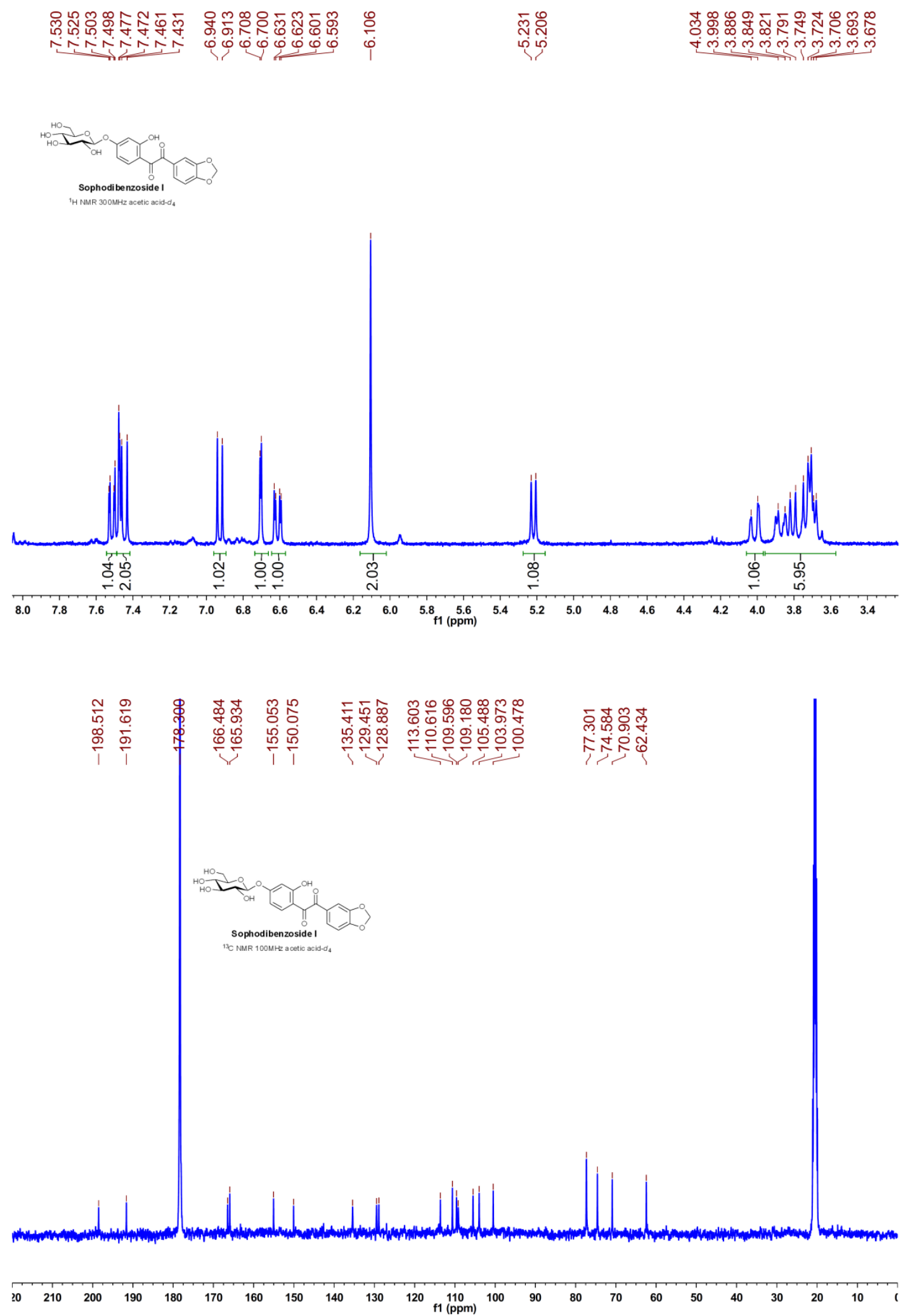